UCRL-LR-154609

\title{
Laser Program Annual Report 1983 Excerpts
}

\author{
J. D. Lindl \\ September 1, 1984
}

U.S. Department of Energy

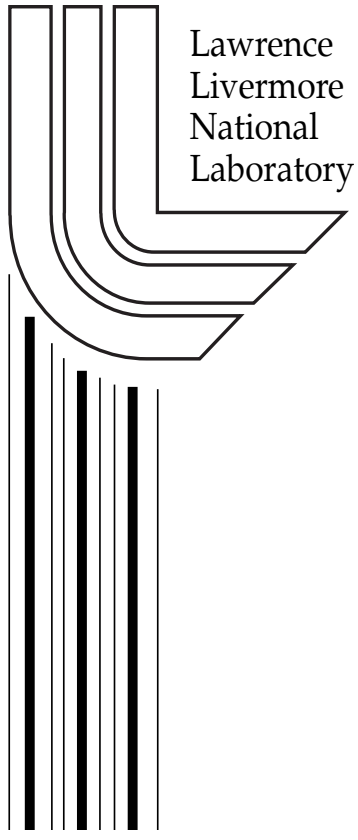

Approved for public release; further dissemination unlimited 


\section{DISCLAIMER}

This document was prepared as an account of work sponsored by an agency of the United States Government. Neither the United States Government nor the University of California nor any of their employees, makes any warranty, express or implied, or assumes any legal liability or responsibility for the accuracy, completeness, or usefulness of any information, apparatus, product, or process disclosed, or represents that its use would not infringe privately owned rights. Reference herein to any specific commercial product, process, or service by trade name, trademark, manufacturer, or otherwise, does not necessarily constitute or imply its endorsement, recommendation, or favoring by the United States Government or the University of California. The views and opinions of authors expressed herein do not necessarily state or reflect those of the United States Government or the University of California, and shall not be used for advertising or product endorsement purposes.

This work was performed under the auspices of the United States Department of Energy by the University of California, Lawrence Livermore National Laboratory under contract No. W-7405-Eng-48.

This report has been reproduced directly from the best available copy.

Available electronically at http://www.doc.gov/bridge

Available for a processing fee to U.S. Department of Energy

And its contractors in paper from

U.S. Department of Energy

Office of Scientific and Technical Information

P.O. Box 62

Oak Ridge, TN 37831-0062

Telephone: (865) 576-8401

Facsimile: (865) 576-5728

E-mail: reports@adonis.osti.gov

Available for the sale to the public from

U.S. Department of Commerce

National Technical Information Service

5285 Port Royal Road

Springfield, VA 22161

Telephone: (800) 553-6847

Facsimile: (703) 605-6900

E-mail: orders@ntis.fedworld.gov

Online ordering: http://www.ntis.gov/ordering.htm

OR

Lawrence Livermore National Laboratory

Technical Information Department's Digital Library

http://www.llnl.gov/tid/Library.html 
Scientific Editor: Charles D. Hendricks

Publication Editors: Peter W. Murphy

M. Louise Rufer

MS date: September 1984

\section{Excerpts}

\section{Laser Program Annual Report 83}

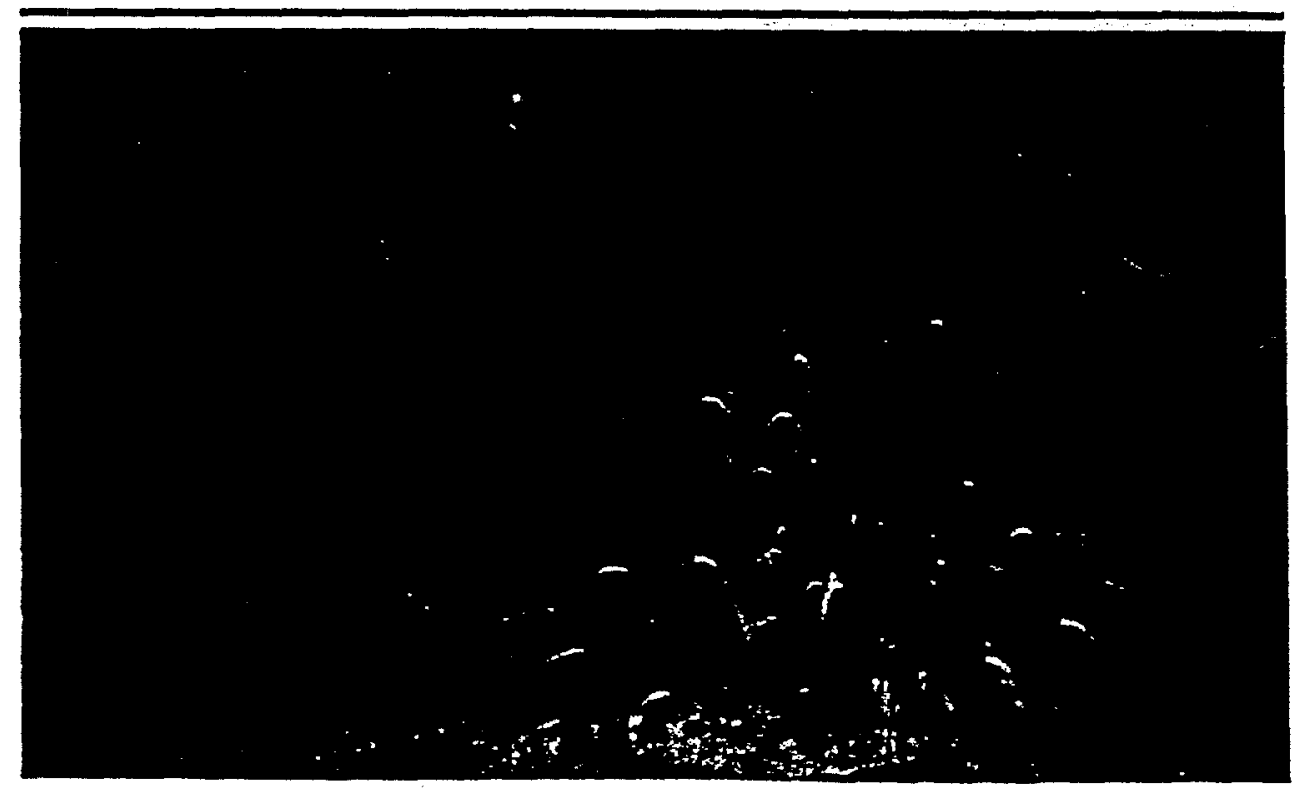

Lawrence Livermore National Laboratory

University of California • Livermore, CA $• 94550$ 


\section{Excerpts From The 1983 ICF Annual Report}

Pages 2-17 through 2-20

Pages 2-28 through 2-35

Pages 2-40 through 2-46

Pages 2-52 through 2-55

Pages 4-1 through 4-48

Some of these pages have been cited as unpublished references in the Book "Inertial Confinement Fusion" by John D. Lindl. They are reprinted here for the convenience of the reader.

"Inertial Confinement Fusion" citations that pertain to the 1983 Annual Report are:

Chapter 2, references 54, 55, 56.

Chapter 4, reference 2.

Chapter 5, reference 6.

Chapter 6, reference 3.

Chapter 11, reference 4. 
Target Design

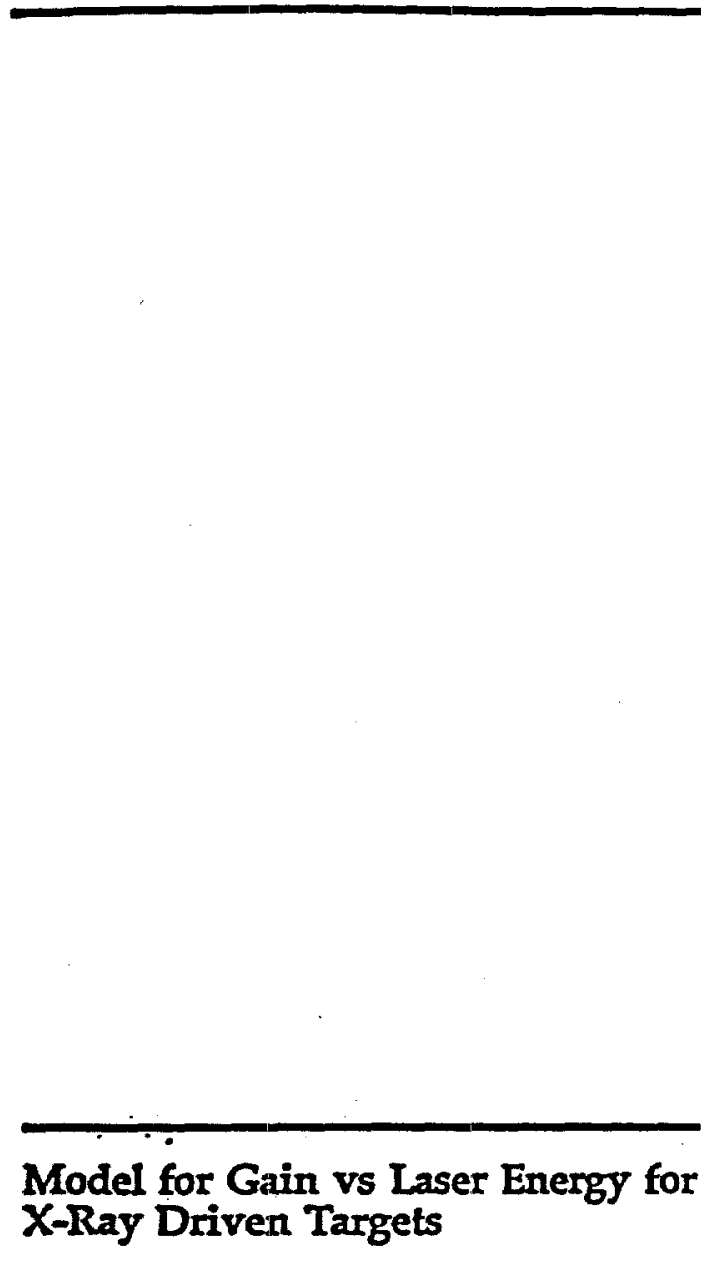

Introduction. In this article we present, from first principles, a simple model for gain vs driving laser energy of classified a ICF targets. We will compare our predictions with results from LASNEX simulations and show good agreement between the two approaches. Our theoretical model borrows heavily from material presented in the undassified 1983 Laser Annual. 2120 Starting from inside the capsule (and later proceeding outurard), we find an optimal gain for an assembled DT fuel of internal energy $\eta E$, assembled at an efficiency $\eta$, assuming energy $E$ was absorbed by the capsule. (Later we will find the laser energy required to achieve this $E$, taking into account hohlraum absorption, conversion to soft $x$-rays, and wall loss vs target-ball absorption.) The optimal gain is given by

$$
G^{*}=6 \times 10^{3} \eta\left(\pi E / \alpha^{3}\right)^{03}
$$

where $\eta E$ is in $M J$ and $\alpha$ represents the degree to which the fuel is off the Fermidegenerate adiabat. The mass in this

\begin{tabular}{cccc} 
r/P & $G / \sigma$ & $M / M^{*}$ & $V / V_{\text {knq }}$ \\
\hline 10 & 10 & 1.0 & 1.0 \\
2.0 & 0.8 & 1.0 & 1.0 \\
3.0 & 0.6 & 1.0 & 1.0 \\
4.0 & 0.3. & 0.66 & 1.2 \\
45 & 0.1 & 0.33 & 1.7 \\
\hline
\end{tabular}

optimal system is

$M^{*}=29(\eta E)^{1.2} \alpha^{-0.6} \mathrm{mg}$.

and the implosion velocity required to bring the fuel into this state is at least

$V_{\text {Imp }}=2.6 \times 10^{7}\left(\eta E / \alpha^{3}\right)^{-0.1} \mathrm{~cm} / \mathrm{s}$.

where we have set $0.5 \mathrm{M}^{*} V^{2}=\eta E$.

These quantities do not vary significantly if the optimal hot-spot radius $(r=63 \mu \sqrt{\eta E})$ is doubled, dropping $G^{*}$ by only $20 \%$, essentially preserving $M^{*}$ and $V_{\text {imp }}$ and at the same time relaxing convergence ratio constraints by a factor of 2 . In Table 2-1, we denote the effect of having $r / r^{*}$. increase. from 1 to 4.5. This will be used later when convergence ratio presents itself as the major constraint to assembling the fuel.

In the following discussion, our goal will be to work towards an expression for the efficiency, $\eta_{c}$ as a function of energy scale, $\eta E$. Thus, combining that with Eq. (2) and, if need be, Table 2-1 (if convergence ratios become a major.constraint), we can find $G=G(B)$ for classified ICF targets.

Radiation-Driven Rocket. The target implodes via a rocket-like reaction of the blowoff plasma driven by soft $x$-ray ablation. This radiation-driven rocket was explored in great detail in Refs. 23 and 24. From LASNEX simulations, we find that the usual rocket equation [ $V_{\text {payload }}=$ $V_{\text {exhusst }} \ln X$, where $X=m / m_{0}$ is the remaining rocket (payload + remaining fuel) mass fraction] takes the following form:

$V=0.33 \sqrt{T_{\mathrm{keV}}} \ln \mathrm{X} \mathrm{cm} / \mathrm{sh}$

This is obviously of the proper form since the exhaust velocity scales as an isothermal sound speed at the driving blackbody temperature, $\tau$. The coefficient accounts for the many complexities due to non-Planckian and multifrequency photonic effects. Since our rocket must reach the terminal velocity
Table 2-1. The effect on the gain $G$ and mass $M$ of the system of having the hot spot radius 8 langer than the optimal value $r^{*}$.

(1)


required by Eq. (4), we find the required ablation fraction

$\ln X=-\frac{0.79}{\sqrt{T_{\mathrm{keV}}}}(\eta E)^{0.1}$.

An important quantity to compute is the in-flight-aspect-ratio (IFAR) of the target as it implodes. Again, we follow the approach of Ref. 22. The shell, on the nearly Fermidegenerate adiabat $P=2 \times \rho^{5 / 3} \mathrm{Mbar}$, is in pressure contact with the radiation ablation driving pressure

$P=2.5 \times 10^{4} T_{\mathrm{keV}}^{3.5} \mathrm{Mbar}$,

yielding an expression for its density, $\rho$

$\rho=288 T_{\mathrm{keV}}^{21}$.

In addition, we reach terminal velocity when the shell has moved in to $r / 2$

$$
\begin{aligned}
0.5 r & =\int V d t \\
& =0.33 \mathrm{v} \bar{T} \frac{m_{0}}{\dot{m}}[1-X(1-\ln X)],
\end{aligned}
$$

where $\ln X$ is given by Eq. (6). To find the thickness $\Delta X$ of the shell, we note the ablation rate

$\dot{m}=5 T_{\mathrm{keV}}^{3}$.

Thus,

$$
\begin{aligned}
\Delta X=\frac{\dot{m}}{\rho} t_{\text {burnthru }} & =\frac{\dot{m}}{\rho} \cdot \frac{m_{0}}{\dot{m}} \\
& =\frac{5}{288 T_{\mathrm{keV}}^{0.9}} \frac{m_{0}}{\dot{m}} .
\end{aligned}
$$

where we have made use of Egs. (8) and (10). Combining Eqs. (9) and (11) yields

$\frac{r}{\Delta X}=\frac{40}{T^{0.4}}[1-X(1-\ln X)]$.

This complicated equation for IFAR can be approximately fit by a power law:

$$
\text { IFAR }=\frac{70}{T_{\text {kev }}^{0.75}} \frac{1}{\left(n E^{0.05}\right.} .
$$

Once IFAR is known, we can find an expression for the original radius of the capsule. First, we define $\mu$ as the ratio of tamper mass to DT fuel mass. Because the total payload mass $M_{f}(1+\mu)$ is $X$ times the original mass, we obtain

$$
\begin{aligned}
\frac{(1+\mu) M_{\mathrm{f}}}{X}= & 4 \pi r_{0}^{2} \rho \Delta r=\frac{4 \pi r_{0}^{3} \rho}{\text { IFAR }} \\
& =0.5 r_{0}^{3} T_{\mathrm{keV}}^{2 \pi}(\eta E)^{0.05} .
\end{aligned}
$$

where we have used Eqs. (8) and (13). Using $M_{f}$ from Eq. (3), and $X$ from Eq. (6), we can find $r_{0}$ if we know $\mu$. This quantity will be discussed next. Summarizing,

$$
r_{0}=\frac{0.4(\eta E)^{0.38}}{T_{\mathrm{keV}}^{0.9}}\left(\frac{1+\mu}{X}\right)^{1 / 3} \mathrm{~cm} \text {. }
$$

Preheat Shield. It would be inaccurate to assume that a viable target had no pr left over in the pusher. This left over tamper must act as a photon-preheat shield, thus keeping the fuel on a low adiabat. Based on LASNEX calculations, we take the required shielding to be of the order of

$(\rho r)=2 \times 10^{-3} \mathrm{~g} / \mathrm{cm}^{2}$.

To simplify the analysis, we presume that this is sufficient and/or necessary for all scales of energy. We can then find the quantity $\mu$, which is the ratio of preheatshield mass to DT mass:

$$
\mu=\frac{4 \pi r_{D T}^{2}\left(2 \times 10^{-3}\right)}{4 \pi r_{D T}^{2}\left(0.2 \Delta r_{D T}\right)}=\frac{10^{-2}}{\Delta r_{D T}} .
$$

We assume the DT shell has an aspect ratio of the order of 10 . Thus,

$\mu=0.1 / r_{\text {o(DT) }}$.

As a crude estimate for $r_{0(D T)}$, we set

$M_{f}=4 \pi r_{0}^{2}\left(0.1 r_{\alpha(D T)}\right)^{(0.2)}$,

and get $M$ from Eq. (3). This yields

$r_{\alpha D T)}=0.5(\eta E)^{0.4}$.

yielding

$\mu=0.2(\eta E)^{-0.4}$.

Recall, however, from Table 2-1, that depending on our choice of off-optimal 
Target Design

assembled fuel conditions, Egs. (20) and (21) will change by $\left(M / M^{*}\right)^{1 / 3}$.

Rocket Efficiency. We define the rocket efficiency as

$\eta=\frac{0.5 m V^{2}}{\sigma T_{t}^{4}}$

Using Eq. (5) for $V$, we find (since $\sigma=1$ in jerk/sh)

$$
\begin{array}{r}
\eta_{\mathrm{H}}=\frac{0.5\left(m_{0} X\right) 0.1 T_{\mathrm{keV}} \ln ^{2} X}{T_{\mathrm{keV}}^{4}\left(m_{0} / \dot{m}\right)(1-X)} \\
=\eta_{\mathrm{D}} \frac{X \ln ^{2} X}{1-X}
\end{array}
$$

where

$\eta_{0}=\frac{\dot{m}}{20 T^{3}}=\frac{5 T^{3}}{20 T^{3}}=\frac{1}{4}$

using Eq. (5).

There is, however, one other effect. We are using as our payload $(1+\mu) M_{\mathrm{r}}$. If there is little or no coupling of tamper kinetic energy into internal DT energy, then the hydro efficiency of interest is

$\eta=\frac{\eta_{H}}{I+\mu}$.

LASNEX calculations confirm that very litthe coupling does occur, so Eq. (24) is justified. Analytic treatments, with a realistic equation of state for typical tamper materials, confirm this as well, but we omit them here for the sake of brevity.

Later we will be concerned with wall loss vs ball absorption, and an expression for the pulse length, $\tau$, will be required. To find $\tau$, we set

$\tau=\frac{m_{0}}{\dot{m}}(1-X)=\frac{\rho_{0}(\Delta r)(1-X)}{5 T^{3}}$.

For a typical beryllium ablator with a 10-to-1 aspect ratio, and a driving temperature of about $250 \mathrm{eV}$, we obtain

$\tau=\left(2.5 r_{0}\right)(1-X)$ sh

Since $r_{0}$ is given by Eq. (14), $r$ can be found.
Other Efficiency Factors. Up to this point, we have been dealing with the energy absorbed by the capsule. There are (at least) three efficiency factors we must consider in converting the incident laser energy $E_{L}$ into absorbed capsule energy $E$. The first is absorption of light by the hohlraum where a $90 \%$ absorption is quite feasible and, in fact, may be too pessimistic and conservative an assumption. Second, is the conversion of absorbed laser light into soft $x$-ray drive. Experimentally, we have thus far achieved conversion efficiencies of $60 \pm 10 \%$ for $3 w$ light. Theoretically, there may be methods (mixed materials, low density foams) that could raise this to a $90 \%$ value. Recent $4 \omega$ experiments may indeed have achieved those values. For the sake of definitiveness, we take the product $f_{\text {abs }} \cdot f_{\text {conv }}$ $=0.8$. Third, we must compute how much of the soft $x$-rays are absorbed by the target ball as compared to wasted energy absorbed by the hohlraum walls. The wallloss formula is approximately

W.L. $=0.5 T_{\mathrm{heV}}^{3} \tau_{\mathrm{ns}}^{0.5} A_{\mathrm{W}\left(\mathrm{mm}^{2}\right)} \mathrm{hJ}$,

where the low- $Z$ ablator of the ball absorbs energy approximately as a blackbody:

B.L. $=T_{\mathrm{heV}}^{\mathrm{t}} T_{\mathrm{ns}} A_{\mathrm{B}\left(\mathrm{mm}^{2}\right)} \mathrm{hJ}$.

At a given driving temperature $(T=2$ to $2.5 \mathrm{heV}$ ) and a given ratio of wall-to-ball area $(9$ to 25), we can find the ball-loss to wall-loss ratio, which will depend on $\tau^{0.5}$ and therefore on $\eta E$. Summarizing,

$\frac{\text { B.L. }}{\text { total energy }}=\frac{1}{\left.1+1 / 2\left(r_{W} / r_{B}\right)^{2} \frac{1}{T \sqrt{t}}\right]}$.

Examples. We assume the target ball is driven at about $250 \mathrm{eV}$, thus insuring a reasonably low IFAR of 35 to 50 . We refer to Table 2-2 for details of the
Table 2-2 Various quantities derived from equations in the text, as the energy scale $\mathrm{E} E$ (in MT) is varied.

\begin{tabular}{llccccccc}
\hline$\eta E$ & $X$ & $\begin{array}{c}V \\
(\mathrm{~cm} / \mathrm{sh})\end{array}$ & $\eta_{H}$ & $\mu$ & $\eta=\frac{\eta_{H}}{1+\mu}$ & $\begin{array}{c}r_{0} \\
(\mathrm{~cm})\end{array}$ & $C R$ \\
\hline 1.0 & 0.2 & 0.26 & 0.17 & 0.2 & 0.14 & 0.46 & 73 \\
0.1 & 0.13 & 0.33 & 0.16 & 0.5 & 0.11 & 0.23 & 115 \\
0.01 & 0.08 & 0.41 & 0.14 & 1.2 & 0.07 & 0.12 & 190 \\
0.001 & 0.04 & 0.52 & 0.10 & 3.1 & 0.03 & 0.07 & 366 \\
\hline
\end{tabular}


Fig. 2-21. Effect of couvergence ratio constraints on gain. $G^{*}$ is optimal gain.

Table 2-3. Various quantities derived from equations in the text, as the energy scale $\boldsymbol{\nabla} E$ (in $M]$ is varied.

Fig. 2-22 Gain vs laser energy. Solid curve is theory discussed in this article. Circles are LASNEX calculations.

Target Design

calculations. For each energy scale, $\eta E$ (in MJ), that we choose, Eq. (6) yields $X$ and then, via Eq. (4) or (5), a payload velocity $V$. Once $X$ is known, Eq. (23) yields $\eta_{H}$ Eq. (21) predicts $\mu$. Therefore, Eq. (24) yields $\eta$, Eg. (15) yields $r_{0}$ and dividing by $r^{*}=63 \mu$ $\sqrt{\eta E}$ gives the convergence ratio (CR). Note the huge $C R$ values at low $\eta E$. Keeping $C R$ at 100 or 50 requires increasing the hot spot radius, $r$. This affects $V$, and therefore

$X$ and $\eta_{c}$ as well as $G / G^{*}$ as per Table 2-1. Figure 2-21 shows how the convergence ratio constraint lowers the achievable gains as we go to low $\eta E$ values.

In Table 2-3, we complete the calculation by assuming that the laser light is $0.35 \mathrm{~mm}$, with a comfortable (from a symmetry point of view) wall-to-ball radius ratio of 4 . The pulse length $\tau$ is derived from Eq. (26). The energy on the capsule is simply $\eta E / \eta$. The total radiation energy is derived from Eq. (29). The laser energy is assumed to be
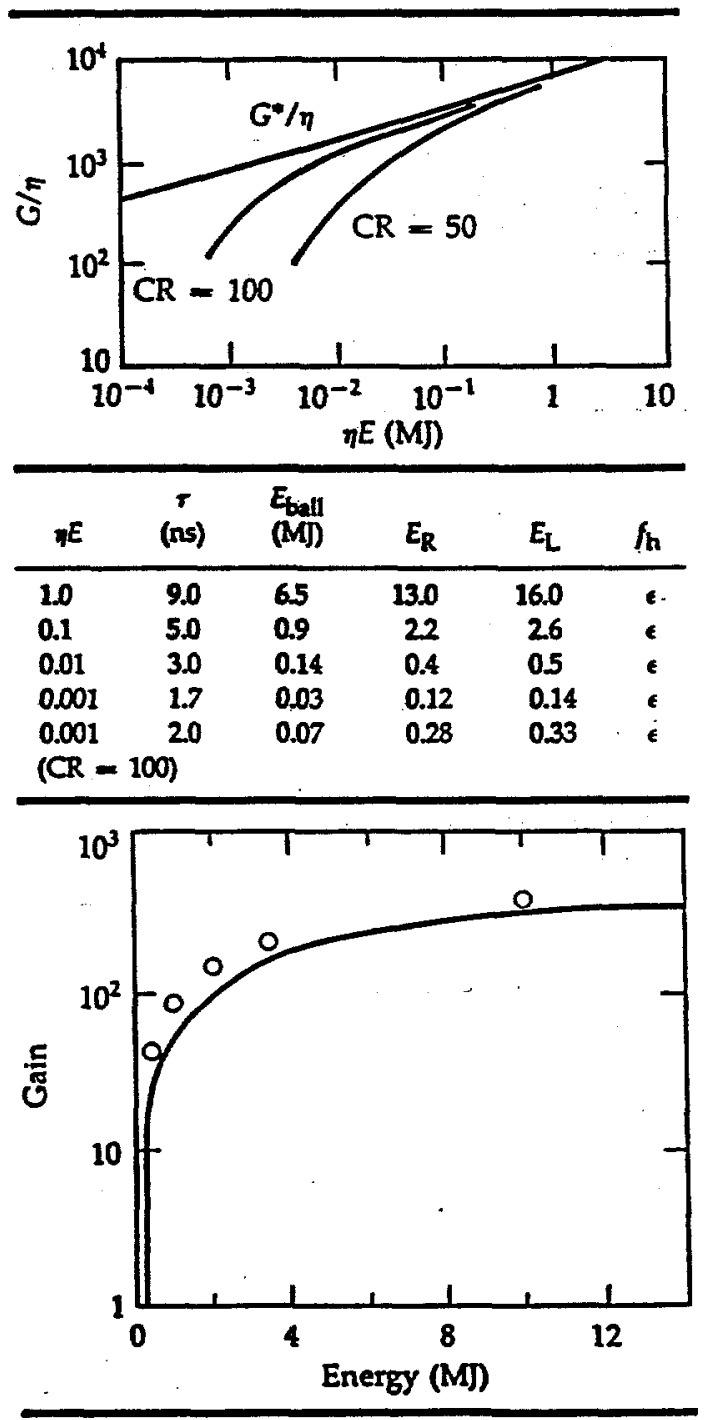

$20 \%$ greater than the radiation energy to account for absorption and conversion inefficiencies. We see that these hohlraums are large enough so that for 0.35- $\mu \mathrm{m}$ light, the scaling laws for $f_{h}$ (percent of laser energy in hot electrons) predict miniscule $f_{h}$ fractions.

The gains vs energy can now be given explicitly, as it is in Fig. 2-22. Note the excellent agreement with the LASNEX calculations.

As a definite example consider $330 \mathrm{~K}$ of laser energy. Assuming we can turn $80 \%$ of that into radiation energy within a hohlraum gives us $280 \mathrm{~K}$, of which 70 will be absorbed by the capsule. For a convergence ratio of 100 the efficiency will be $1.5 \%$, yielding $1 \mathrm{KJ}(\eta E=0.001)$ in the assembled DT fuel. From Eq. (2) we can expect $G_{\text {capsule }}=\left(6 \times 10^{3}\right) \eta(\eta E)^{0.3}=11$. But, due to the convergence ratio constraint (see Fig. 2-21), $G=0.3 \times G^{*}$, or 3.3. The actual gain is $(70 \mathrm{KJ} / 330 \mathrm{KJ}) \times G_{\text {capsule }}$ or a gain of 0.7. On the other hand, at a laser energy of $16 \mathrm{MJ}$, similar considerations led to an $E_{\mathrm{R}}$ of $13 \mathrm{MJ}$, an $E_{\text {capsule }}$ of $6.5 \mathrm{MJ}$, an $\eta$ of $15 \%$, leading to an $\eta E$ of $1 \mathrm{MJ}$ in the assembled DT fuel. Thus, Eq. (2) leads to a $G^{*}$ capsule of 900 and an actual gain of $(6.5 / 16)$ $\times 900=365$.

Conclusions. We have, from first principles, derived the gain vs laser energy for classified. ICF targets. The nature of the gain cliff in the $E_{\mathrm{L}}<1 \mathrm{M}$ ) range stems from a number of phenomena. As we go down in scale, the required implosion velocity increases, leading to lower hydro efficiency. The preheat shield takes up more and more of the payload, thus leading to a very low effective efficiency. With shorter pulse lengths (for shorter-scale targets), more energy is absorbed by the wall than by the target. For small scales; the convergence ratio grows quite large, requiring that the assembled fuel configurations be nonoptimal (with lange hot spot regions) leading to . even lower gains. All these effects conspire to produce the diff in gain at $1 \mathrm{MJ}$ or below. To defeat this limit would require either ultra-smooth symmetry, double-shell targets (if mixing can be defeated), or polarized fuel, which would lower ignition criteria and increase burn efficiency, leading to less stringent requirements on velocities and convergence ratios, and yield higher gains.

Authors: M. D. Rosen and J. D. Lindl 
Design Studies of Single-Shell ICF Capsules for Nova

Introduction. One of the main objectives of the Nova program is to attempt to demonstate, with the Nova laser, a capsule inplosion that is hydrodynamically equivalent to that of a langer capsule appropriate for a full-scale ICF reactor. Specifically, that means that the initial DT fuel configuration should be geometrically similar to that of the reactor capsule with all lengths scaled down by the same scale factor, and that the implosion should proceed in times scaled down from the reactor capsule by the same factor. If this hydrodynamic similarity of the implosion is approximated, the Nova capsule should achieve the same final densities, pressures, and temperatures as the reactor capsule just prior to ignition, but in a geometrically scaled-down configurationagain by the same factor The idea is to experimentally address, as soon as possible, many of the hydrodynamic issues involved in the ablative implosion of an ICF reactor capsule.

In general, it is not possible to achieve a hydrodynamically equivalent target with one that is purely geometrically scaled. The ablator material, for example, has a natural scalelength set by its Planck mean free path, $\lambda_{p e}$ (Ref 33) that will determine the density scale height at the ablation front. Since hydrodynamic instability is governed by the ratio of $\lambda_{p} / \Delta r$, where $\Delta r$ is the shell thiekness, we must increase the ablator opacity at smaller sizes to maintain hydrodynamic similarity. Also, the density gradient scalelength between the hot spark plug and the dense main fuel during stagnation of the implosion is set by thermal conduction, which does not scale geometrically. For the same temperature hot spot, a Nova capsule would have a sthallower density gradient.
The challenge, then, is to find a

Nova capsule design that meets the

following criteria.

- The fuel configuration should be a direct scale of a successfully simulated reactor capsule design.

- The fuel configuration should be diagnosable both before the implosion and at peak density.

- The capsule must adequately contain the fuel under experimental conditions prior to implosion.

- The ablator must couple efficiency, at this smaller size, to thermal radiation in the $200-\mathrm{eV}$ range so that the fuel will achieve the implosion velocity, $0_{\&} \approx 0.3 \mathrm{~cm} / \mathrm{sh}$, required for ignition at reactor scale.

- During the implosion, the fuel must remain on the low adiabat expected for the larger reactor capsule.

- The ablator composition must be chosen to have stability characteristics at the ablation front similar to reactor capsules.

- The overall design, generally, must minimize the potential for hydrodymic instability.

So far our focus has not been on obtaining final designs. Rather, we have attempted to gain insight inito the dynamics expected at Nova scale so that early decisions could be made on the choice of target fabrication technologies (especially, choices of ablator materials), and also to set some of Nova's operational requirements for such variables as energy and pulse shape. To this end, we have used the LASNEX code to simulate Nova capsule implosions.

Investigation Path. We have considered two generic capsule designs, shown in Fig. 2-35. Type I is a simple sphere of ablator material surrounding a shell of solid (ayogenic) DT that in tum is filled with low-density DT gas at a density appropriate to near equilibrium with the solid at cryogenic temperatures. The type II design incorporates the additional feature of a strong high-Z "mandrel" that can have many functions; it can be a pressure vessel for the DT at warmer temperatures, a uniform substrate on which to deposit ablator material, a shield to inhibit fuel preheat during the implosion, or simply an ablation stop. In each case, the capsule is suspended in a hohlraum about 3.5 times the capsule diameter. The dimensions shown are those of two particular designs (capsules $B$ and $H$ in Table 2-4) appropriate for a total laser input 


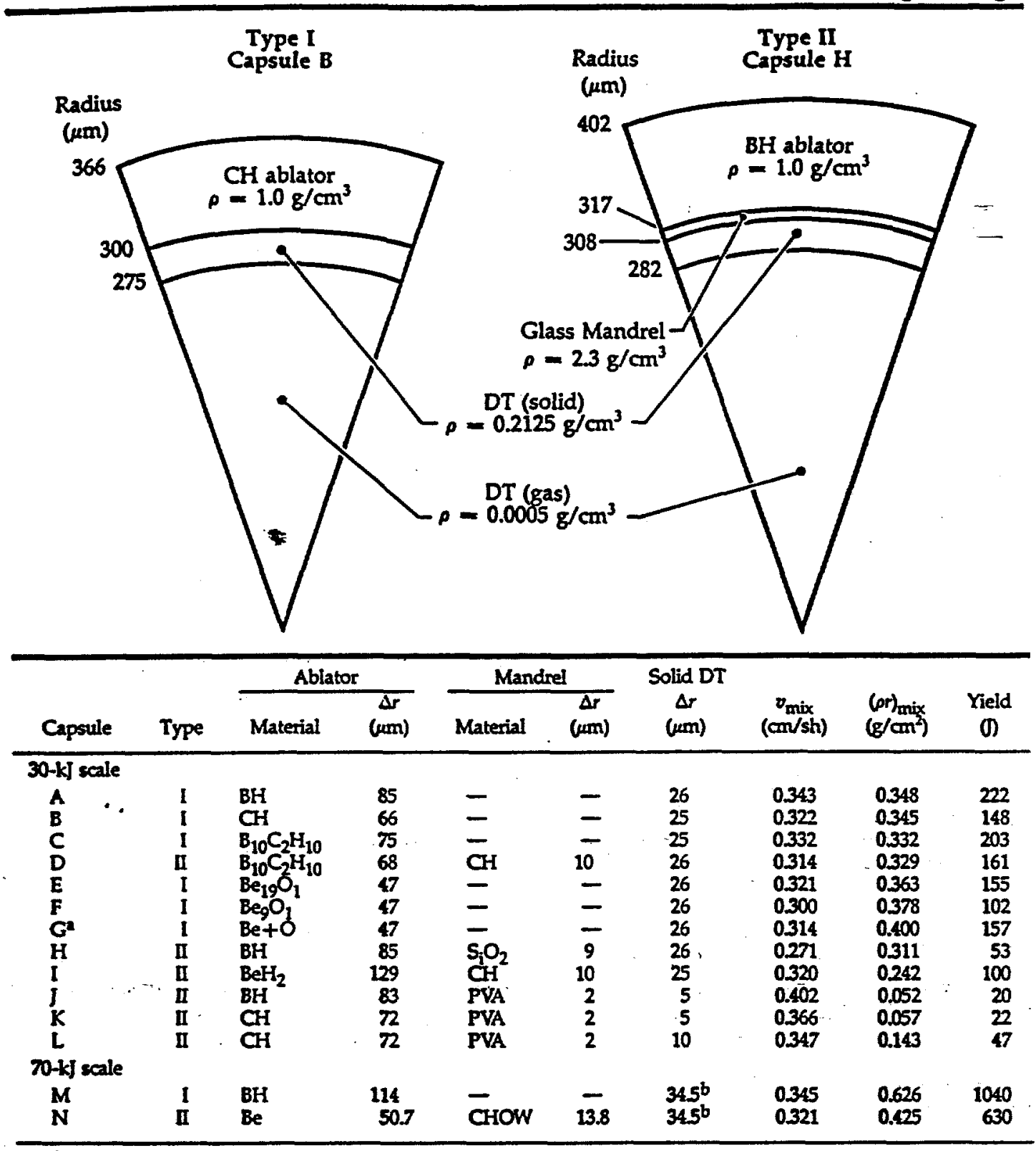

Fig. 2-35. Scale drawings of two generic designs for Nova ICF capsules (capsules $B$ and $H$ in Table 2-4) appropriate for $30 \mathrm{~kJ}$ of input laser energy.

Table 2-4. Design and performance parameters for potential Nova ICF capsules.

See Fig. 2-40.

buel outer radius is $414 \mu \mathrm{m}$.

energy of 30 to $40 \mathrm{~kJ}$ of $3 \omega_{0}$ light. (A range of input energies is given because of uncertainty in the conversion efficiency of laser energy into $x$ rays in Nova-scale hohlraums.) The fuel configuration is approximately a direct scale of that in a reactor capsule. ${ }^{34}$ We have considered two input energies (at 3w) appropriate for Nova, 30 to 40 and 70 to $90 \mathrm{~kJ}$. Capsules at the larger scale are a factor of $\approx(70 / 30)^{1 / 3}$ or $\approx 1.3$ larger.

We have then proceeded in the following way when determining capsule design. The fuel and (if required) mandrel configuration are fixed by the scaling and by whatever is physically required of the mandrel. With the laser energy fixed, and with the requirement that the main radiation drive be in the 200- to $230-\mathrm{eV}$ range, then hohlraum diameter is also fixed. Previous experiments combined with scaling arguments show that this radiation temperature, $T_{r}$ regime represents the best compromise between too much preheat by hot electrons on the one hand (higher $T_{r}$ ), and too much potential for hydrodynamic instability on the other (lower $T_{\text {f }}$ ). To be considered then, an ablator material must couple to this radiation well enough to generate the ablation pressure required for a good, $v_{f}>0.3$ $\mathrm{cm} / \mathrm{sh}$, implosion. This eliminates materials, such as $\mathrm{LiH}$, at the low- $\mathrm{Z}$ end that are too 
transparent to $200-\mathrm{eV}$ radiation at this scale, and materials at the higher- $Z$ end, such as glass or plastics with significant amounts of oxygen, that develop an albedo near unity at this scale and become very inefficient. Left are intermediate- $Z$ materials such as polymers of $\mathrm{BH}, \mathrm{CH}$, or $\mathrm{B}_{10} \mathrm{C}_{2} \mathrm{H}_{10}$ metallic beryllium, or $\mathrm{BeH}_{2}$. These, in fact, are the materials we have considered. For a given ablator material, the ablator thickness and laser pulse shape are adjusted to achieve a good implosion velocity without either ablating so much that the fuel also is ablated, or so little that too high a $T_{\mathrm{r}}(>230 \mathrm{eV})$ would be required. Finally, the laser-energy input is fine-tuned so as to achieve the highest possible final fuel pr. At that point, it is appropriate to look carefully at that particular design to evaluate how well it meets the requirements for fuel velocity, low adiabat, and low potential for instabilities, and to look for design flaws and possible improvements.

Table $2-4$ is a summary of the design and performance of the capsules we have considered up to now. For the 30-kJ capsules, the solid fuel outer radius was 300 or $308 \mu \mathrm{m}$. For 70-kJ capsules, it was $414 \mu \mathrm{m}$. We have characterized the fuel implosion velocity and compression by giving the
Fig 2-36. Input bser power and resultant hohlraum radiation temperature used for expeule G.

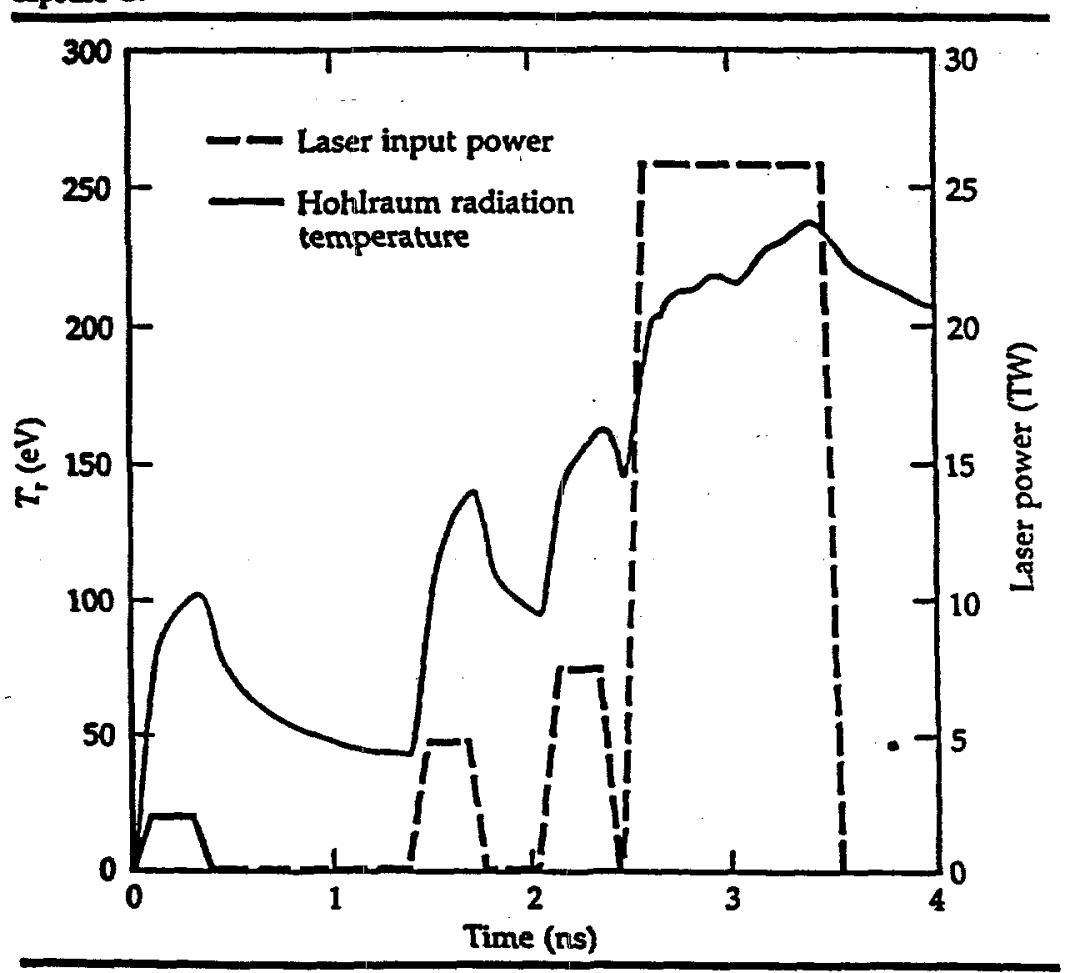

fluid velocity at the fuel-ablator/mandrel interface and the fuel $\int p d r$, "pr," at the socalled "mix" time. (This is when a fluid element at the fuel-ablator/mandrel interface, if it never decelerated, would have reached 0.8 of the radius it had when it actually did begin to decelerate at stagnation.) Last, we give the nuclear yield in joules. (One joule is equivalent to about $3.6 \times 10^{11}$ neutrons.) It should be noted that these capsules do not really ignite; their hydrodynamic behavior is unaffected by thermonuclear bum simply because at these scales, an energetically insignificant amount of $\alpha$-particle energy is generated and coupled to the fuel.

The results of our investigations are discussed further in this article.

Pulse Shaping. The objective of pulse shaping is to bring the driving pressure on the fuel up to a high value without generating too much entropy in the fuel by shocks. In practice, it is found that with four or more shocks the fuel can be brought up to the required pressure ( $\sim 50$ to $80 \mathrm{Mbar}$, corresponding to $\sim 100$ to 120 Mbar in the ablator) without departing too much from its original adiabat. Fewer shocks generate too much entropy. (We shall say more about this entropy issue later in this article.) For these studies, we have adopted a "picket fence" pulse shape, as most appropriate for Nova, in which the laser energy is applied as four separate bursts of increasing laser power. The first three generate pulses of thermal radiation in the hohlraum and drive blast waves into the capsule, while the last and most energetic pulse is long enough to drive the ablative implosion. In Fig. 2-36 we show the laser input power and resulting hohlraum radiation temperature for a $30-\mathrm{kJ}$ capsule (G) characteristic of the designs we have studied. We have assumed trapezoidal pulses of 100-ps rise and fall times.

Ideally, the four shocks generated would coalesce very near the inner edge of the solid fuel. This puts a small mass of fuel, the originally gaseous fraction, on a high adiabat so that it can serve as an effective spark plug at peak compression, while leaving the vast majority of the fuel in a low adiabat to be compressed to maximum pr. Furthermore, such timing means that the 
Target Design

highest pressure is applied at maximum fuel-shell volume so that maximum $p d V$ work can be done to accelerate the fuel. If shocks converge in the dense fuel, then some of it will be shock-heated too strongly. If, on the other hand, a shock breaks out too far ahead of the next, then the succeeding shock goes down the resulting density gradient behind the first, again resulting in strong shock heating. Morever, in this latter case, volume for $p d V$ work has been wasted. The low-entropy, highvelocity requirements then set constraints on pulse-timing and pulse-height errors. We have found that timing errors of $<30$ ps are generally tolerable and are within the experimental designs. Sensitivity to amplitude variations has not yet ben well established.

Fuel Adiabat. So-called "preheat" of the fuel in some designs can spoil the implosion by decreasing the fuel compressibility, thus decreasing its final density when the implosion stagnates. We have attempted to look in some detail at this effect and to assess quantitatively the importance of the various sources of preheat in particular designs. Then, by determining which features of the design are responsible for significant preheat, we can either modify them or eliminate the particular designs from consideration altogether.

First, we wish to stress the point that it is not the addition of heat $(d Q)$ that determines the fuel compressibility, it is rather the addition of entropy ( $d s=d Q / T)$. It makes a very big difference when the heat is added because the fuel temperature varies during the implosion by some seven orders of magnitude. Too strong a first shock can be much more damaging than photon heating late in the implosion when the ablator is almost burned away, even though much more heat is dissipated in the fuel by the photons than by the shock.

To see how compressibility changes with the addition of entropy, we have used the local equation-of-state tables for DT to generate a family of isentropes in the pressure vs density plane. Some of the lower members of the family are shown in Fig. 2-37. The numbers labeling the curves also give the specific entropy along that isentrope, relative to the initial cyogenic value, in units of $0.1 \mathrm{jerk} / \mathrm{keV} \cdot \mathrm{g}$, i.e.,

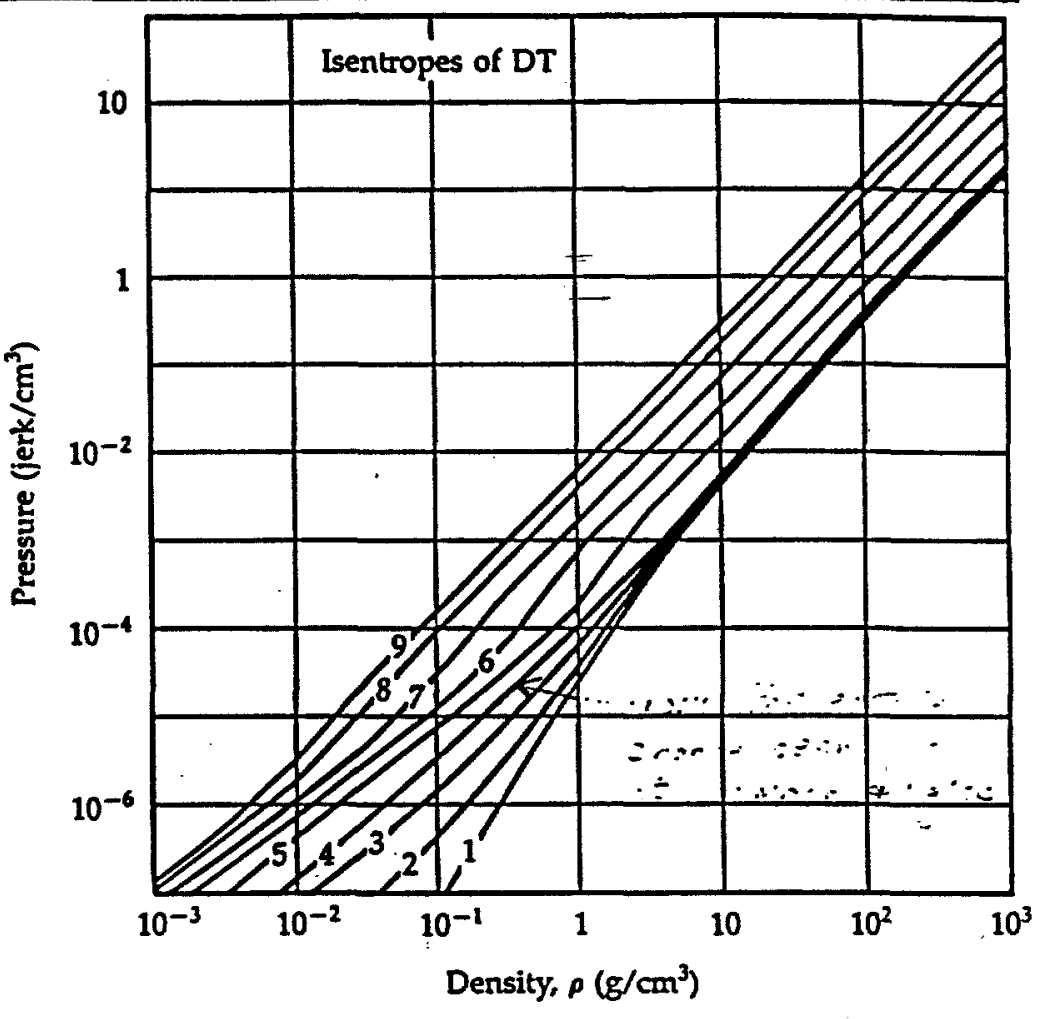

along curve 3 we have the specific entropy, $s=0.3 \mathrm{jerk} / \mathrm{keV} \cdot \mathrm{g}$. Since only changes in entropy are meaningful, we have, for converience, put $s=0$ when $T=11.6 \mathrm{~K}$ and $\rho=0.2125 \mathrm{~g} / \mathrm{cm}^{3}$.

Keeping in mind that we wish to compress the fuel above $100 \mathrm{~g} / \mathrm{cm}^{3}$, we note the following features of the curves shown.

First, for $p \geq 5.0 \mathrm{~g} / \mathrm{cm}^{3}$, curves $1,2,3$, and 4 are seen to nearly coalesce. This coalescence is just above the so-called Fermi degenerate adiabat where the pressure is due almost entirely to the degeneracy pressure of the electrons $\left(k T_{e} \leqslant \epsilon_{\text {Fermi }}\right)$. (For $\rho<5.0 \mathrm{~g} / \mathrm{cm}^{3}$, the $\Delta s=0$ adiabat curves down from $P \propto \rho^{5 / 3}$ because the state of DT at these densities and low temperatures, being dominated by atomic and molecular binding, is poorly approximated by a Fermi gas.) Second, there is a fairly abrupt transition at $\Delta s \approx 0.4$. For $\Delta s<0.4$, the adiabatic compressibility is a very weak function of $s$. However, above that threshold the compressibility decreases exponentially with. increasing entropy:

$$
\begin{aligned}
& x_{s}=\frac{1}{\rho(\partial \rho / \partial P)_{s}} \sim \rho^{-5 / 3} \\
& \times 10^{-3 . \pi \pm s-0.41} \quad \Delta s>0.4 \text {. }
\end{aligned}
$$

Fig. 2-37. Isentropes for a 50/50 DT mixture. The numbers on the curves give the change in specific entropy, in units of 0.1 jerk/ keV. $\mathrm{g}$, from its value in the solid. cryogenic 6 $0.2125 \mathrm{~g}_{\mathrm{cm}} \mathrm{cm}^{3} \mathrm{~T}=$ $11.6 \mathrm{~K})$ state. 
Tanget Design

Our designs therefore have a natural margin of $\Delta s \leqslant 0.4$. The idea then is to exploit this margin to the fullest without going too far.

By keeping track of fuel temperatures and fuel heating/cooling rates during LASNEX simulations of capsule implosions, we can post-process the output to reconstruct the specific entropy of the fuel divided into its constituent sources as functions of time during the implosion. Characteristic results of doing this are shown in Fig. 2-38 for two designs under consideration-capsules B and J of Table 2-4.

There are four important entropy sources (sinks) in our simulations-shocks, photon heating (cooling), electron conduction heating (cooling), and electron-ion coupling. (Heating by hot electrons is another potentially important source, but being dependent on too much unknown physics, it has been neglected in this survey study. Current estimates are that it will be a small effect with $3 \omega_{0}$ laser light at these radiation temperatures.)

In Fig. 2-38 the running integrated entropy from each source, averaged over the originally solid fuel, is plotted vs time, along with the total $\Delta s$. The curves stop just prior to peak compression. Capsule B, Fig. 2-35, represents reasonably good design ( $\Delta s<0.5$ while it matters). Capsule I represents something of a disaster $(\Delta s \approx 0.67$, but an illustrative disaster.

Capsule $\mathrm{J}$ is of the second type described in Fig. 2-35, and modified in the following ways. Its solid DT shell still has a radius of
$300 \mu \mathrm{m}$ but is only $5 \mu \mathrm{m}$ thick. The mandrel is a 2- $\mu \mathrm{m}$-thick shell of PVA plastic, and the ablator is an 83- $\mu \mathrm{m}$ shell of BH polymer. This design was not originally considered as a counterexample, but rather as representative of a high-efficiency design that would be relatively easy to fabricate (though not precisely a hydrodynamically equivalent target). Indeed, the low fuel mass and efficient $B H$ ablator result in an implosion speed of $0.4 \mathrm{~cm} / \mathrm{sh}$, but the poor fuel adiabat results in a final fuel density only about one tenth that of capsule B $\left(50 \mathrm{~g} / \mathrm{cm}^{3}\right.$ vs $500 \mathrm{~g} / \mathrm{cm}^{3}$ ).

We have generated diagrams similar to Fig. 2-38 for all our designs and can make some general remarks. Capsules B and J are characteristic of the extremes.

Shocks clearly represent the largest source of entropy. Indeed, the requirement of obtaining an implosion velocity of $v_{\mathrm{f}} \approx 0.3 \mathrm{~cm} / \mathrm{sh}$ means that we must use up almost our entire margin of $\Delta s=0.4$ with the four shocks in our.pulse-shaping scheme in order to do enough $p d V$ work on the fuel to obtain this fuel speed. Other things being equal, an additional picket (one more shock) added to the pulseshaping scheme could be quite advantageous. The first shock is the worst offender in the present scheme-typically using up $90 \%(\Delta s \sim 0.36)$ of the margin by itself Some preliminary calculations using the equation-of-state tables indicate that if the pressure jump in this shock were divided into two smaller jumps, these could give a total $\Delta s<0.27$, without lengthening the
Fig-2-38. Changes in opecific entropy due to various sources, aVeraged over the originally solid fraction of the DT fuel, as functions of implosion time for capsules B and $J$. The time axis begins just before shocks start to arrive at the fuel and ends fust before peak compression.

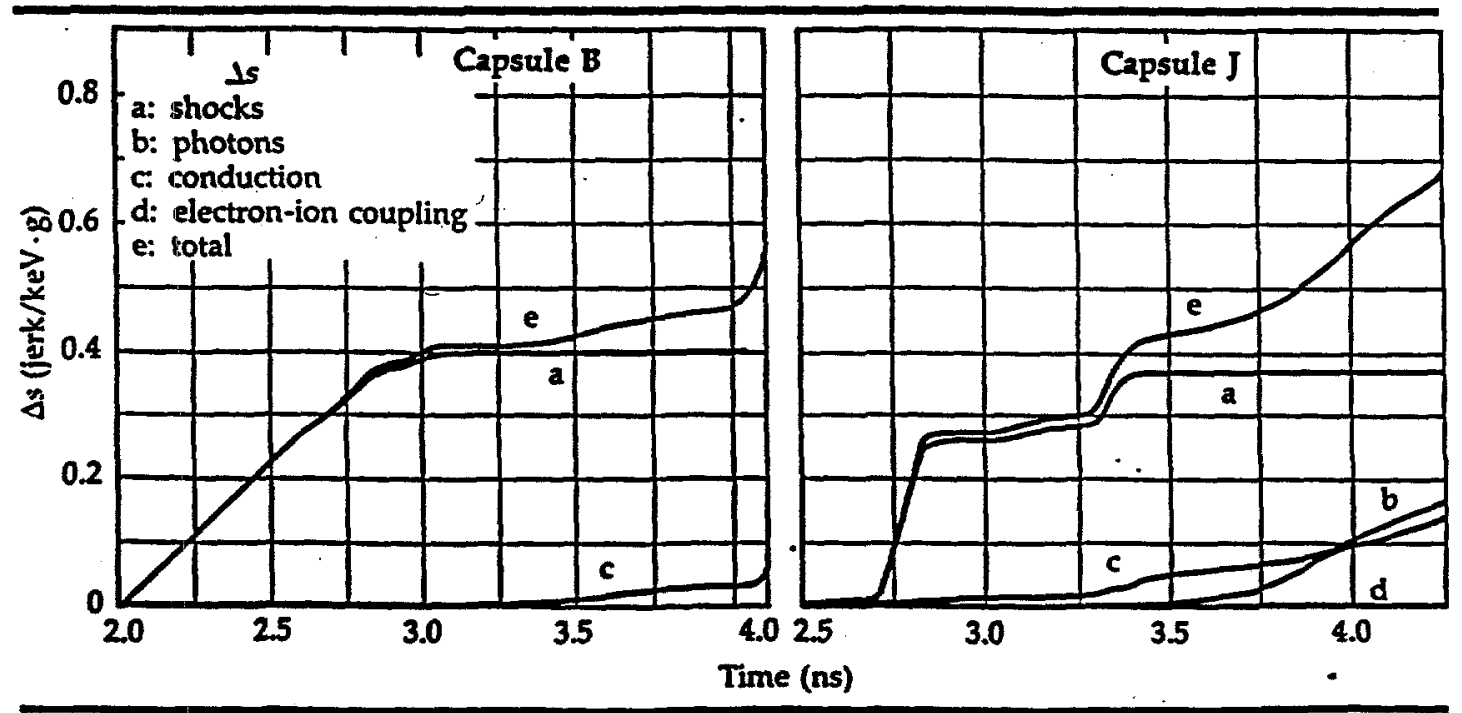


Target Design

time of the total pulse train to more than 10 ns. It is apparent that fewer shocks would be disadvantageous.

In both capsules, photon and conductive entropy sources are comparable with each other and are smaller than shocks. But since shocks have used up the margin, they are quite significant and represent areas of potential design improvement. The electron and ion temperatures apparently stay sufficiently close to each other that coupling between them is not a significant total entropy source. However, there could be more complications because the isentropes of Fig. 2-37 assume that the electron temperature equals the ion temperature.

Closer examination in both cases shows that the damaging conductive flux is inward from the ablator in capsule B and from the PVA mandrel in capsule J (not outward from the hot, low-density fuel). In both cases, the matter just outside the fuel couples more efficiently to the radiation field and gets hotter than the fuel. But in capsule J, the configuration of less opaque BH over very opaque PVA (29. at.\% carbon and 14 at.\% oxygen) results in very strong heating of the thin PVA layer (leading to a high temperature) right next to the thin layer of DT. Because conductive heating penetrates approximately like $t^{1 / 2}$, its effect on specific entropy averaged over a thin layer can be much worse than averaged over a thick one. The thin, opaque PVA has another damaging effect-it converts hard photons, which have penetrated that far and which would couple poorly to DT, into softer photons that it reemits into the fuel and that couple much more strongly to it. This is essentially why the photonic entropy source is some three times larger in capsule J than in B. It is straightforward to improve the design-use more opaque $\mathrm{CH}$ as the ablator and a thicker layer, $10 \mu \mathrm{m}$, of DT (such as in capsule L). And although such a capsule is less efficient, $v_{\mathrm{f}} \approx 0.35 \mathrm{~cm} / \mathrm{sh}$, it achieves three times the compressed fuel pr (0.14 vs 0.05 ). It is better yet to use a styrene mandrel and $25 \mu \mathrm{m}$ of DT and end up effectively with capsule $B\left(v_{f} \approx 0.32 \mathrm{~cm} / \mathrm{sh}\right.$, $\rho r \approx 0.35 \mathrm{~g} / \mathrm{cm}^{2}$ ).

Potential for Hydrodynamic Instability. Our focus here has been on RayleighTaylor (R-T) instabilities in which the pressure and density gradients are opposed, and a low density fluid attempts to accelerate a higher density fluid ahead of it. Since our studies have, so far, been limited to 1-D simulations, we are unable to say much about instabilities driven by shocks, with their characteristic phase reversals, or about the nonlinear evolution of the R-T instability in our designs. Rather we have attempted to find out where and why problems are likely to occur, to use a very simple model of the dispersion relation for rough estimates of the magnitude of the effect, and to look for design strategies to minimize instability growth.

Performance of the Nova capsule designs we have studied, is vulnerable to R-T instability at three places. The first is the low entropy/high entropy fuel interface at stagnation, leading to mix of cold and hot fuel. Instability here cannot be significantly alleviated, within the context of the general designs of Fig. 2-35, except by minimizing instability elsewhere that could seed the instability here, so we shall say no more about it. The second potentially unstable place is the fuel-ablator or fuel-mandrel interface. This region develops an adverse density configuration because when hard $x$-ray photons penetrate to this region, they heat the higher opacity ablator or mandrel material much more efficiency than they heat the DT, leading to a temperature jump from cold DT to warm ablator/mandrel. Pressure continuity across the interface then enforces an adverse density jump as warm ablator/mandrel expands against cold DT. The result is the sort of density profile shown in the top half of Fig. 2-39, in which we show density vs radius snapshots taken at two characteristic times during the implosion of a type-I capsule with a beryllium (95 at.\%) and oxygen (5 at.\%) ablator, capsule E. We see that the shell of solid DT has become a density spike followed by a trough of lower-density, warmer material. The third area of potential instability is at the ablation front itself, where the pressure is reaching its peak value in the How at a radius langer than the radius of. peak density.

At this point, some general remarks about the capsule implosions we have studied are in order. Because the fuel shells in a given series all start at about the same radius ( $300 \mu \mathrm{m}$ for $30-\mathrm{kJ}$ tangets), and because 


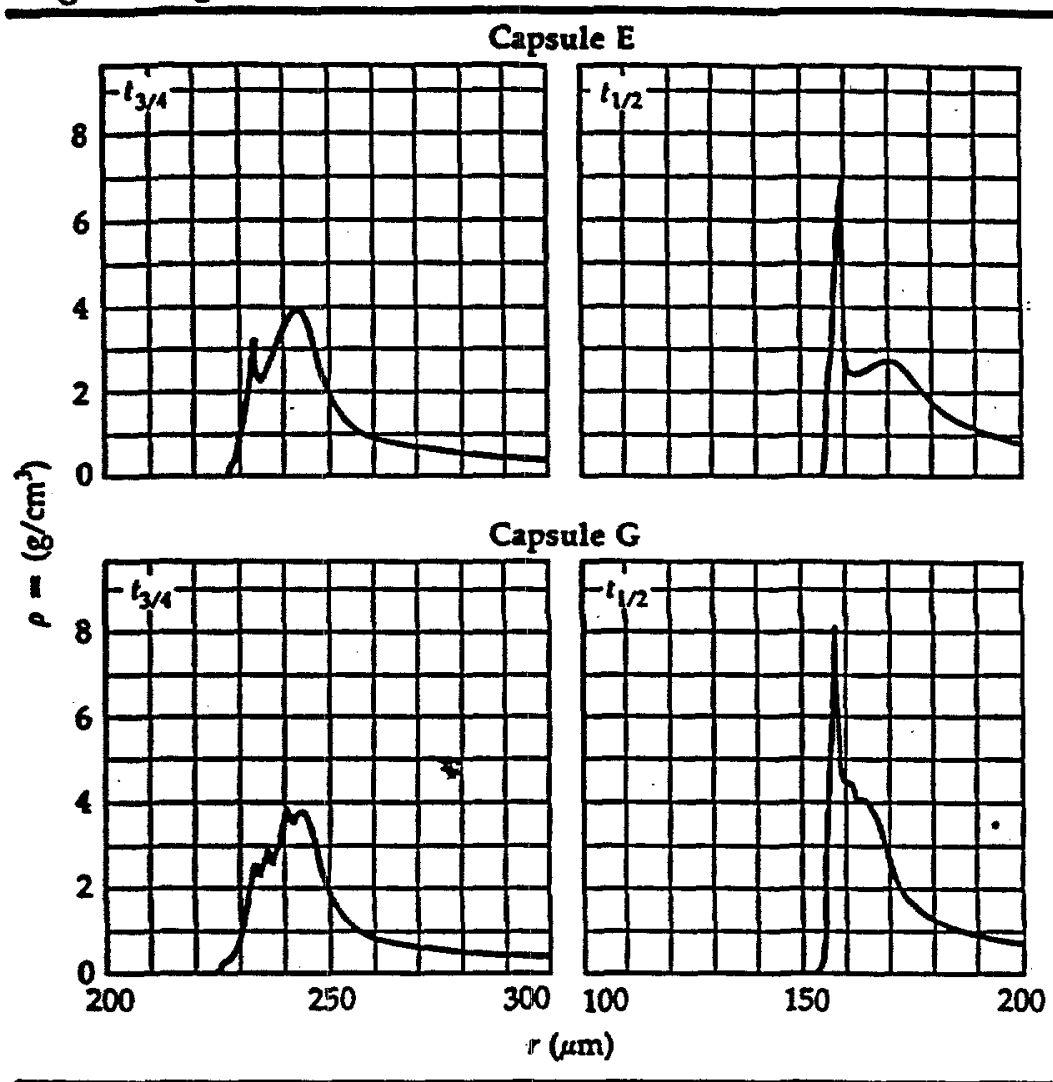

Fis 2-39. Density vs ridius profiles for copsules $E$ and $G$ at times in the implosion when the DTablator/mandrel interface is at $3 / 4$ and $1 / 2$ its original radius. the design goal is roughly the same final velocity, $v_{\mathrm{f}} \approx 0.3 \mathrm{~cm} / \mathrm{sh}$, the average fuel acceleration does not vary over too wide a range. Then, if the laser pulses are properly timed so that the resulting shocks break out of the solid fuel in tight sequence, the acceleration of the fuel turns out to be smooth and roughly constant rather than impulsive. For comparing designs, it is useful, under the circumstances of roughly constant acceleration from time-to-time and from capsuleto-capsule, not to try to analyze each implosion as a whole but simply to look at potential instability growth at a few representative times during the implosion. We have chosen the times when the DT-ablator/ mandrel interface is at $3 / 4$ and $1 / 2$ its original radius, which we call $t_{3 / 4}$ and $t_{1 / 2}$, respectively. (For constant acceleration these are the times when the implosion is $1 / 2$ and $3 / 4$ over, respectively.)

The form of the dispersion relation for gas-dynamic flows such as these is as yet unknown. Insofar as there is a straight density jump at the DT-ablator/mandrel interface, the growth rate might be expected to be roughly classical, but there will be some stabilization because the density will be smoothly varying. If the density had an exponential profile, the growth rate would be given by (Ref. 35)

$$
\gamma^{2}=8 \frac{k \beta}{k+\beta} \frac{\rho_{h i}-\rho_{l o}}{\rho_{h i}+\rho_{l 0}},
$$

where $g$ is the fluid acceleration, $k$ is the perturbation wave number, and $\beta$ is the reciprocal of the density scale height. The growth rate given above saturates at $\gamma \rightarrow \sqrt{g \beta}$ for $k \gg \beta$ and $\rho_{h i} \gg \rho_{10}$. Adopting this dispersion relation as valid locally leads us to define a sort of local maximum instability growth rate,

$$
\begin{aligned}
\gamma(r) & =\sqrt{g(r) \beta(r)} \\
& =\sqrt{\left(\frac{1}{\rho} \frac{\partial P}{\partial r}\right)\left(\frac{-1}{\rho} \frac{\partial \rho}{\partial r}\right)} .
\end{aligned}
$$

Snapshots of the run of this quantity can then be inspected at our characteristic times to see the location and magnitude of the problems. No pretense is made that this analysis would tell us anything quantitative in an absolute sense. It is, however, useful for comparison. Of all the capsules we have looked at (before attempting to find a strategy to ameliorate the problem), all but one had the largest rate, by factors of 2 to 10 , at the DT-ablator/mandrel interface compared to the ablation front. Moreover, stability at the ablation front is expected to be enhanced by such effects as "fire polishing" and convection of unstable flow downstream-stabilizing effects not present at the inner interface. Clearly, design strategy should concentrate on that inner region.

One strategy is suggested by the exception noted above, which was precisely the type II capsule of Fig. 2-35, capsule H. The mandrel can be made. so optically thick that virtually no photons reach the fuel-mandrel interface. However, the instability naturally pops out elsewhere, at the mandrel-ablator interface where hard photons attempt to drive an inner ablation front into the glass against a back pressure of colder, denser BH. Still, the unstable region is no longer right next to the fuel and has a lower growth rate (characteristic of an ablation front) subject to further stabilizing. effects. The main problem with this capsule is that the load of glass, which is a poor ablator, makes the capsule somewhat inefficient, $v_{\mathrm{f}} \approx 0.27 \mathrm{~cm} / \mathrm{sh}$. 
Target Design

A second strategy is suggested by the idea that if the instability is due to a sudden inward decrease in the opacity at the fuel-ablator interface, perhaps the effect could be minimized by decreasing the opacity in gentler steps. Furthermore, if the ablator opacity is first increased inward, the outer regions will still be most efficiently ablated while the photon spectrum reaching the inner regions will be sufficiently hardened that photon-matter coupling will be reduced. This opacity tailoring could be most easily accomplished by doping the ablator with a higher- $Z$ element. A possible realization of such a capsule, capsule $G$, is shown in Fig. 2-40 in which a beryllium ablator is doped with oxygen. When this capsule is imploded, it develops a much more Eenign density profile. Figure 2-39 is a direct comparison of density profiles for two beryllium ablator capsules, E and G, at our two characteristic times. On the bottom half are the profiles developed by the capsule of Fig. 2-40. On the top half are the profiles for a capsule identical in every respect except that the oxygeri doping was constant at 5 at.\% throughout the ablator. The improvement at the $t_{3 / 4}$ point is obvious. The growth rate is dramatically reduced over much of the implosion time. Moreover, the unstable interfaces within the ablator could probably be virtually eliminated by smooth doping variations. Later, at the $t_{1 / 2}$ point, this strategy has a much smaller effect because the strategic opacity variations have been ablated away. Nevertheless, the Atwood number at the fuel-ablator interface is still seen to be somewhat reduced. Finally, we should mention that this capsule, $G$, achieved the highest pr $(0.40)$ of all of the $30-\mathrm{kJ}$ capsules we considered in spite of a relatively modest implosion speed $\left(v_{f} \approx 0.31\right)$. The reason should be clear from our discussion in the previous section and the discussion above-the ablator next to the fuel stays cool enabling the fuel to stay on the lowest possible isentrope. The main design problem with this particular capsule design is the difficulty in diagnosing the initial fuel configuration through opaque beryllium. However, the doping strategy should be generally applicable.

For completeness, we tried a reverse doping strategy in which the opacity was increased inward all the way to the fuel-ablator interface. This capsule developed density profiles more adverse than those of either case described above.

Conclusion. Our present strategy for high performance Nova capsules will rely on polystyrene $(\mathrm{CH})_{N}$ capsules overcoated with hydrocarbon (CH). This material has ablation-front stability characteristics very similar to that of proposed high-gain capsules at the multimegajoule size. ${ }^{35}$ The $C H$ overcoat can be doped with higher- $Z$ elements to reduce instability at the fuelablator interface as discussed above. The combination of $\mathrm{CH}$ over a polystyrene mandrel represents the smallest materials development effort over current capabilities for targets that are hydrodynamically equivalent to high-gain capsules. Also, with a very small silicon doping, these capsules are potentially transparent. They can then be characterized by optical techriques, the only ones currently developed that have the precision required for these high-convergenceratio experiments.

Authors: S. P. Hatchett and

J. D. Lindl
Capsule G

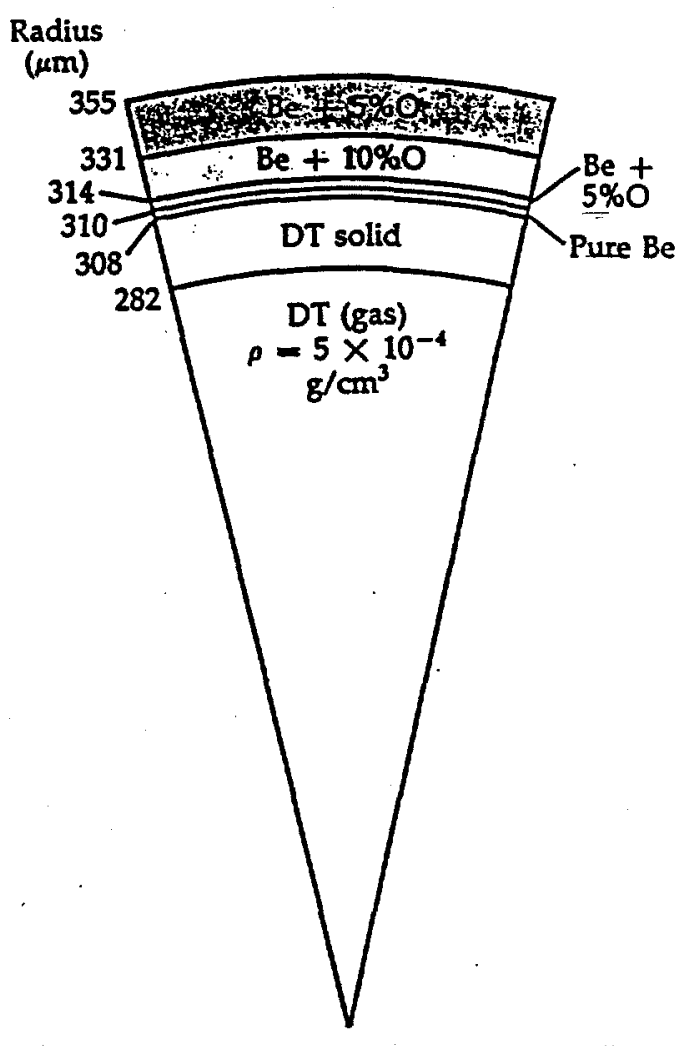

Fig- 2-40. Sale drawing of capsule $G$ showing oxygen doping variations to reduce $\mathrm{R}-\mathrm{T}$ instability. 
Hydrodynamic and PlasmaStability Iimitations on the Choic of Laser Wavelength for Radiation Driven ICF Implosions

There is a trade-off between plasma stabilit and hydrodynamic stability in the implosit of ICF capsules. Higher intensities result in higher pressures, and higher pressures allog use of thicker shells, which reduce the impact of perturbations due to fluid instabilities. However, the higher intensities increa the probability of preheat or of reduced $a b$ sorption due to plasma collective effects. U of shorter wavelength lasers reduces the in pact of these instabilities for both direct- at radiation-driven tangets. We develop in this artide a model for radiation-driven capsule that compares the sensitivity of the requir laser wavelength to each effect; we also discuss the leverage that is achieved either by reduced growth of hydrodynamic perturbations or by increased drive at a given laser wavelength and at a fixed hot electron production. 
Target Design

Hot-electron production in hohlraums comes from the wall plasma when laser light strikes the high- $Z$ radiation case, and from a volume interaction with plasma that is confined in the hohlraum and that eventually builds to a significant fraction of critical density.

Experiments with high- $Z$ disks to investigate the properties of the wall plasma have generally shown a level of hot electrons acceptable for high-gain targets. Current research is focusing on accessing longer scalelengths, which will be characteristic of reactor targets, to see if this favorable trend will continue.

However, hohlraums can have much higher levels of hot electrons than an open geometry target with the same intensity if the blowoff plasma produces long scalelengths of underdense plasma. The plasmafilling model ${ }^{\text {to }}$ developed on Shiva and Argus provides an estimate of the amount of energy that can be put into a hohlraum as a function of size, wavelength, and pulse length before filling problems begin. Experiments on Novette have shown hot-electron levels somewhit below this model, but it still serves as a useful guide. Using this model, we have, for Gaussian pulses,

$f_{\text {hot }}=0.5 \exp \left(-\frac{A^{2}}{15 \lambda^{2} E \tau}\right)$,

where $f_{\text {hot }}$ is the fraction of the laser energy golng into hot electrons, $A$ is the hohlraum area in $\mathrm{mm}^{2}, \lambda$ is the wavelength in microns, $E$ is the laser energy in kilojoules, and $r$ is the pulse length in nanoseconds. The fraction of hot electrons tolerable by a high-gain target depends on their energy and on where they are produced. We can tolerate about $2 \mathrm{eV}$ of preheat in the fuel of a typical high-gain target; this corresponds to about $10^{-4}$ of the laser energy delivered to the capsule. Typical multimegajoule targets are self-shielding against electrons with energies less than $100 \mathrm{keV}$, so that only electrons in the tail of the distribution penetrate to the fuel for typical hot-electron temperatures. Also, if the hot electrons are produced near the case, the capsule will only intercept a small fraction of the electrons because it subtends a small solid angie because of these factors, most target designs can tolerate $1 \%$ or more of the laser light being converted to hot electrons. If we take $f_{\text {hot }}=0.01$, we require

$\frac{A^{2}}{15 \lambda^{2} E \tau}=3.92$

for all designs. For a given $A, \lambda, E$, and $\tau$ we use the Marshak model developed by Rosen $^{41}$ and others to calculate the temperature that is achieved consistent with this hot-electron fraction,

$T_{\mathrm{r}}(\mathrm{heV})=2.70\left(\frac{\epsilon E}{A \sqrt{\tau}}\right)^{1 / 3}$,

where $\epsilon$ is the fractional conversion of laser light to $x$ rays.

Equation (39) was developed for Gaussian pulses, while high-gain targets have a temporally shaped pulse with either a long, low-intensity foot or a series of short, highintensity pickets followed by the main drive pulse. Plasma blows into the hohlraum during the early part of the pulse although at lower velocity than during the drive pulse. Based on a variety of target designs, we estimate the effective pulse length to be

$\tau \sim 2 E^{1 / 3}$

The conversion efficiency, $\epsilon$, is a function of wavelength, increasing at shorter wavelengths. For intensities of primary interest for high-gain targets, it varies approximately as

$\epsilon=0.33 \lambda^{-0.5}$.

Combining Eqs. (40) to (43) we get

$T_{\mathrm{r}}(\mathrm{heV})=0.75 E^{1 / 18} \lambda^{-0.5}$.

Equation (44) is the temperature possible at a given wavelength and energy consistent with $f_{\text {hot }}=0.01$. Because we are interested in reactor-scale targets, we pick $E=5000 \mathrm{~kJ}$ so that

$T_{\mathrm{r}}(\mathrm{heV})=1.20 \lambda^{-0.5}$.

At any given temperature, only shells that exceed a certain in-flight aspect ratio (IFAR) $r / \Delta r$ can be imploded to an ignition velocity of $3 \times 10^{7} \mathrm{~cm} / \mathrm{s}$. Equation (45) 
must be coupled to an implosion model of radiation-driven ablative implosions to calculate the required IFAR.

High-gain capsules must be imploded in such a way that the fuel remains near Fermi degenerate. This means that a cryogenic shell of fuel will be compressed from its initial density of $0.21 \mathrm{~g} / \mathrm{cm}^{3}$ to a density consistent with the applied pressure so that

$\rho \sim 0.66 P^{0.6}(\mathrm{Mbar})$

for matter at twice the Fermi adiabat near densities of $1 \mathrm{~g} / \mathrm{cm}^{3}$. Since the pulse shape for shells is done in such a way that one reaches peak pressure at very near the initial radius (to minimize the peak.drive pres- . sure), we must use this compressed derisity in calculating the IFAR. The ablator can be more or less dense than the fuel, depending on its compressibility, but in the optimum case the ablator density equals the fuel density. If the ablator is higher in density, the overall shell is thinner than necessary. If the ablator is lower in density than the fuel, there is an unstable region at the fuelablator interface in addition to the one at the ablation surface.

The motion of material driven by subsonic radiation ablation can be described by a rocket equation. We use Hatchett's theory $^{42}$ to obtain for the rocket velocity

$V(\mathrm{~cm} / \mathrm{s})=10^{7} \sqrt{T}(\mathrm{heV}) \ln \left(\frac{m_{0}}{m}\right)$

where $m_{0}$ is the initial mass and $m$ is the payload mass. Similarly; the ablation rate is given by

$\dot{m}\left(\mathrm{~g} / \mathrm{cm}^{2} \cdot \mathrm{s}\right)=\rho V_{\mathrm{a}}=4.7 \times 10^{5} \rho T^{3}$.

The ablation pressure is given by

$P(\mathrm{Mbar})=8 \mathrm{~T}^{3.5}(\mathrm{heV})$.

Using Eq. (46) to obtain the shell density we have

$V_{\mathrm{a}}(\mathrm{cm} / \mathrm{s})=2 \times 10^{5} \mathrm{~T}^{0.9}(\mathrm{heV})$.

These equations are approximately valid for beryllium or other materials, such as appropriately tailored foams, that have similar ablation characteristics. Since the re quired implosion velocity is $3 \times 10^{7} \mathrm{~cm} / \mathrm{s}$. we have

$V_{\mathrm{ign}}=3 \times 10^{7}=10^{7} \sqrt{T} \ln \left(\frac{m_{0}}{\mathrm{~m}}\right)$

or

$\ln \left(\frac{m_{0}}{m}\right)=\frac{3}{\sqrt{T}}(\mathrm{heV})$

at ignition velocity. We require that the shell reach its implosion velocity by the time it is at half its radius because most of the implosion volume is used up by that time. Therefore, we have

$0.5 r=\int_{0}^{t_{1}} V d t$,

where $t_{1}$ is the time at which the condition: of Eq. (51) apply.

If we let

$\frac{m}{m_{0}}=\left(1-\frac{V_{\mathrm{a}} t}{\Delta r}\right)$

we can use Eqs. (45) and (47) to obtain

$$
\begin{aligned}
\frac{r}{\Delta r} & =100 T^{-0.4} \\
\times & {\left[1-\left(1+\frac{3}{\sqrt{T}}\right) \exp \left(-\frac{3}{\sqrt{T}}\right)\right] . }
\end{aligned}
$$

Between 100 and $400 \mathrm{eV}$, this is approximately

$\frac{r}{\Delta r}=90 T^{-7 / 8}(\mathrm{heV})$

or, using Eq. (50),

$\frac{r}{\Delta r}=150 P^{-0.25}(\mathrm{Mbar})$.

The above estimates are obtained with the assumption that the shell mass is at constant density. In fact, there is a pressure ar density gradient across the shell for a shel moving at constant acceleration so that about $90 \%$ of the shell mass is contained 
Target Design

thickness of $\sim 1.5$ times those above. We inil use this latter estimate for the shell Whickness so that

Yxt--

tr $=60 T^{-7 / 8}(\mathrm{heV})$

ser

$$
=100 P^{-0.25} \text { (Mbar) . }
$$

Because the material we are imploding is compressible and must be kept Fermi degenerate, the IFAR depends only weakly on pressure. If the shell were at constant density, we would have $\mathrm{Pr} / \Delta r$ approximately constant instead of the above dependence. Because of the compressibility and because we cannot achieve radiation temperatures much beyond $200 \mathrm{eV}$, we must implode shells whose IFAR exceeds 25. Detailed LASNEX calculations result in the same conclusion. To successfully implode such capsules requires that the growth rate of Rayleigh-Taylor-like modes be reduced substantially below the classical value.

Rosen and Lindl have developed a similar theory for direct-drive capsules. Because of their higher exhaust velocity and lower ablation rate, direct-drive capsules have about a factor-of-2 higher IFAR at a given pressure. Also, the achievable pressure for direct-drive capsules is lower because of plasma physics effects so that the minimum possible IFAR is 3 to 4 times that of a radiation implosion at a similar wavelength.

These estimates are consistent with detailed LASNEX calculations by Pollaine and Lindl for direct drive capsules. (See the following article, "Effect of Capsule Aspect Ratio on Hydrodynamic Efficiency.")

For the classical Rayleigh-Taylor instability, we have a growth rate given by

$\gamma_{R \cdot T}=\sqrt{k a \alpha}$,

where $k$ is the wave number, $a$ the acceleration, and $\alpha=\left(\rho_{2}-\rho_{1}\right) /\left(\rho_{2}+\rho_{1}\right)$ is the Atwood number. If we take $\alpha=1$, corresponding to a large density mismatch across the ablation surface, which is typical of ICF capsules, and assume constant acceleration over a distance equal to $r / 2$, we can integrate Eq. (59) to obtain the number of -foldings, $n$, for a given wave number mode,

$n=\int \gamma d t \sim \sqrt{k r}$, where we ignore the effects of spherical convergence and compressibility. To obtain the number of e-foldings, we have to pick a wave number. Typically, the worst wavelength is chosen to be one about equal to the compressed shell thickness or $k=2 \pi / \Delta r$. Longer wavelengths grow more slowly while shorter wavelengths saturate before penetrating the shell. Using the above wavelength we have

$n_{\mathrm{cl}}=\sqrt{\frac{2 \pi r}{\Delta r}}$.

The maximum tolerable number of $e$-foldings depends on surface finish and shell thickness. A 1-M]-scale target has a compressed shell thickness of about $50 \mu \mathrm{m}$ and the best possible surace finish is probably about $100 \AA$. We penetrate the shell with a growth factor of 5000 or after 8.5 e-foldings. Using Eq. (56) implies that $r / \Delta r<12$. This is a lower IFAR than can be imploded at temperatures accessible to laser-driven ICF capsules. Ignoring the fast-growing short-wavelength modes is also not acceptable because they can modecouple to longer wavelengths to seed their growth. For the classical case with very fast growing short-wavelength modes, Youngs ${ }^{43}$ has investigated this mode coupling. His numerical work, and supporting experiments, suggest a turbulent mixing depth $h$, independent of initial surface conditions

$h=0.05 a t^{2}=0.05 r$

if the acceleration occurs for a distance equal to $r / 2$. His work suggests that $r / \Delta r<20$ to prevent penetration, also a result requiring IFARs that are too low for ICF needs.

To succeed, we must find techniques that reduce the growth rate of Rayleigh-Taylor modes. Because of possible mode coupling, we would like all modes to have a growth rate sufficiently low that none of them suffer more than 4.5 to $7 e$-foldings, depending on surface finish, during the implosion. If we write

$n_{\max }=\sqrt{2 \pi \alpha_{\text {eff }} \frac{r}{\Delta r}}$ 
Target Design

then $\alpha_{\text {eff }}$ which is a measure of the reduced growth, must satisfy

$\alpha_{\text {eff }}<\frac{n_{\text {max }}^{2}}{2 \pi r / \Delta r}$

for a successful implosion. Using Eqs. (45) and (58), we have

$$
\frac{r}{\Delta r}=51 \lambda^{0.44}
$$

Using this in Eq. (63) gives -

$\alpha_{\text {eff }}<\frac{n^{2} \lambda^{-0.44}}{100 \pi}$

The required value of $\alpha_{\mathrm{eff}}$ is plotted in Fig. 2-49 for growth factors of 100, 1000, and 5000. Any successful implosion must result in growth rates below this line. Equation (66) can be satisfied either by going to a short enough laser wavelength or by finding a mechanism that reduces the growth rate of fluid instabilities. Since one only gains as $\lambda^{-0.44}$, there is a high payoff for understanding such mechanisms. Currently, virtually no experimental evidence exists that quantitatively addresses the growth of Rayleigh-Taylor modes at a surface driven by soft $x$ rays.

We will discuss two possible mechanism that are predicted by analytical and numeri cal calculations and that can act either separately or together to produce a signifi. cant reduction in growth for RayleighTaylor modes.

One possible mechanism is density gradi ent stabilization discussed by Lelevier ${ }^{44}$ and Munro. ${ }^{45}$ Since Rayleigh-Taylor modes are) surface modes with a depth $1 / k$, if there is finite density scalelength $1 / \beta$ comparable to $1 / k$, the mode only sees a fraction of the full density difference so that the effective Atwood number is reduced. In the presenc of a finite density gradient, Eq. (59) is replaced by

$$
\gamma=\sqrt{\frac{k \beta}{k+\beta}} a
$$

and the number of $e$-foldings becomes

$$
n=\sqrt{\frac{k \beta}{k+\beta}} r<\sqrt{\beta r} \text {. }
$$

For radiation implosions, a finite density gradient is produced by long-range photon
Fig. 2-49. Finite derlsity gradients and advection can have a large impact on the wavelength or drive required for a successful ICF implosion.

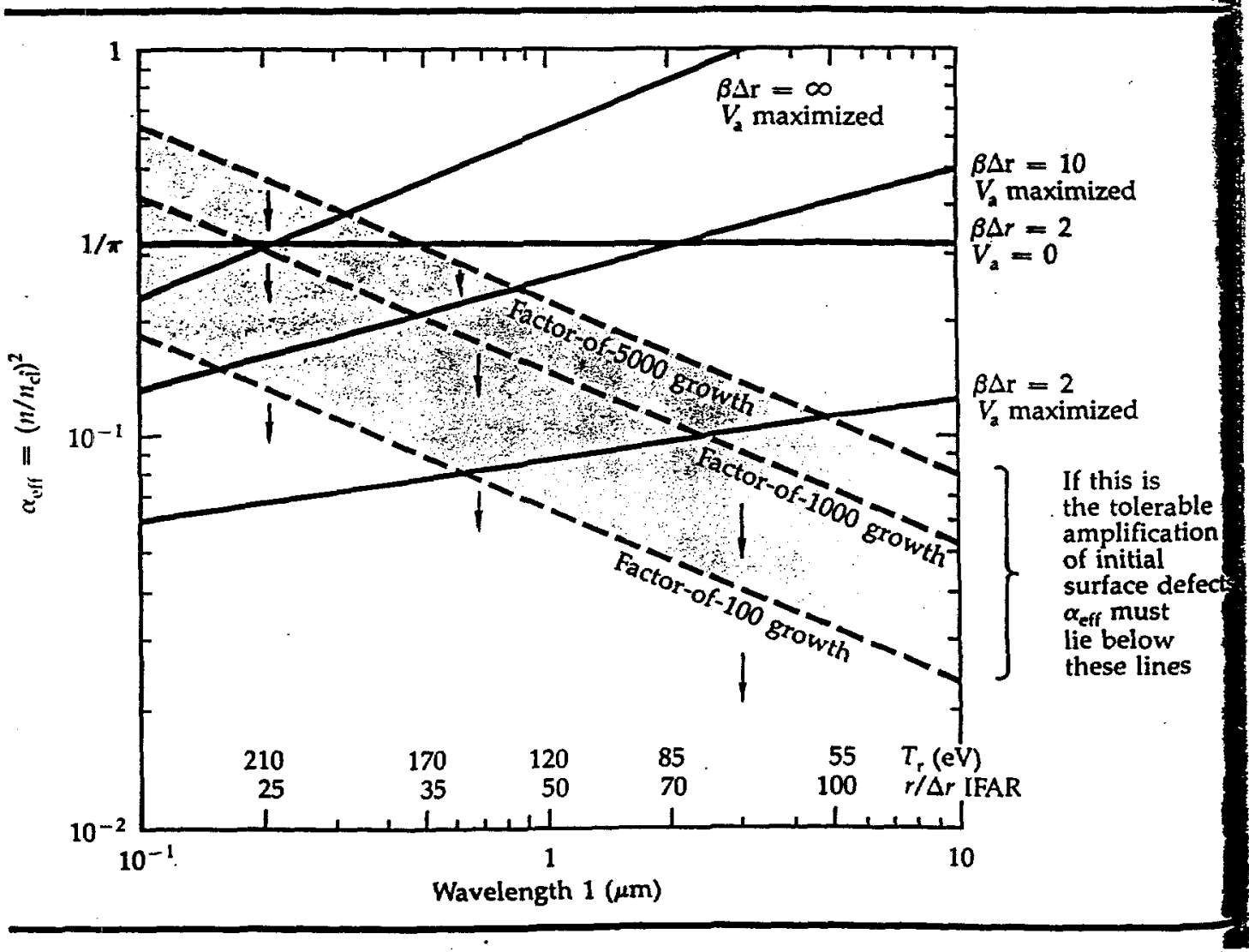


larget Design

in the tail of the distribution that are in the tall inside the ablation front. By adfusting the material composition, this denilty gradient can be adjusted over a wide range. However, a practical limit occurs for $\beta \Delta r \sim 2$. If one tries to achieve longer scalelengths, enough photons penetrate the entire shell that the fuel becomes preheated.

For $\beta \Delta r=2$, we have

$\left(\frac{n_{\max }}{n_{\mathrm{d}}}\right)^{2}=\alpha_{\mathrm{eft}}=\frac{1}{\pi}$.

This effect is plotted in Fig. 2-49 and shows that for wavelengths less than $1 / 3 \mu \mathrm{m}$ we can just reach a low enough $r / \Delta r$ for a successful implosion with the model presented here with a growth factor of 5000. There is no margin of error and the fastest growing mode does have time for 8 e-foldings so the shell will be badly perturbed. The physics of density gradient stabilization is well understood but we do not have any experimental experience with its practical limitations.

Another effect due to ablation, first suggested by Bodner, ${ }^{46}$ also arises due to the surface nature of Rayleigh-Taylor modes.

These modes have the form

$\Lambda=A_{0} e^{r t} e^{-k x}$

so that a surface of constant $A$ moves into a shell of material with the velocity $V=\gamma / k$. If the ablation velocity $V_{\mathrm{a}}$ exceeds this velocity, then the surface is ablated off faster than the mode can grow so that the mode is stabilized. Bodner obtained a growth rate

$\gamma=\sqrt{k a}-k V_{a}$.

In the presence of a density gradient, this growth rate becomes

$\gamma=\sqrt{\frac{k \beta}{k+\beta}} a-k V_{a}$

If we assume, as is true for all highperformance ICF capsules, that about $80 \%$ of the shell is ablated away in reaching the required implosion velocity and that the ablation velocity is a constant in time, the number of $e$-foldings becomes

$n=\sqrt{\frac{k \beta}{k+\beta}} r-0.8 k \Delta r$
By itself, ablation in general has less of an impact than that of optimizing the density gradient. For the case $\beta \Delta r \rightarrow \infty$, so that for all wavelengths of interest $\beta$ drops out of the above formula, the maximum number of $e$-foldings is given by

$n_{\max }=\frac{0.3125 r}{\Delta r}$

so that

$\alpha_{\text {eff }} \sim \frac{n_{\max }^{2}}{n_{\mathrm{cl}}^{2}}=0.64 \lambda^{0.44}$

For laser wavelengths longer than $\sim 0.2 \mu \mathrm{m}$, the ablation rate is not high enough for ablation, or advection, to dominate the effects of densitygradient Stabilizaxion.

However, in combination with gradient stabilization, advection reduces the maximum number of $e$-foldings substantially. The case for $\beta \Delta r=2$ and $\beta \Delta r=10$ are plotted in Fig. 2-49. For these cases, the maximum number of $e$-foldings, $n_{\text {max }}$ and the value of $(k \Delta r)_{\max }$ at which this occurs (for $\beta \Delta r=2$ ) is approximately

$n_{\max }=0.41\left(\frac{r}{\Delta r}\right)^{0.68}$ or $\alpha_{\text {eff }}=0.087 \lambda^{0.158}$,

$(k \Delta r)_{\max }=0.29\left(\frac{r}{\Delta r}\right)^{0.466}$

or, for $\beta \Delta r=10$,

$n_{\max }=0.42\left(\frac{r}{\dot{\Delta} r}\right)^{0.83}$ or $\alpha_{\text {eff }}=0.26 \lambda^{0.29}$,

$(k \Delta r)_{\max }=0.42\left(\frac{r}{\Delta r}\right)^{0.595}$

There is substantial theoretical uncertainty in the dispersion relation given by Eq. (72). For example, Bodner calculated a much different dispersion relation when electron thermal conduction was important for energy transport. However numerical calculations by Mikaelian and by Lindl achieve results that are qualitatively similar to Eq. (65).

In the work of Mikaelian, $\mathrm{CH}$ foils are accelerated by a 1-ns radiation pulse 
Fig. 2-50. The capsules In our LASNEX simulations consisted of a CF ablator and $1 \mathrm{mg}$ of solid DT fuel. Initial fuel aspect ratios were $S=r_{1} /\left(r_{2}-r_{1}\right)$ $-25,5,10$, and 20 .

Fig. 2-51. CH ablator thickness (0) and mass $(\Delta)$ vs $S$. Ablator thickness was chosen so that enough CH remined to shield the DT from radiation at the end of the laser pulse.

\section{Target Design}

characteristic of Novette $2 \omega$ hohlraums. In these calculations, wavelengths shorter than about $0.30 \mu \mathrm{m}$ are stable while longer modes grow slowly. Simple.estimates of where the transition should occur and of maximum growth rates are fairly consistent with Eq. (72). We have found that high-gain capsules composed of wetted foams with compositions optimized to maximize the density scalelength see much less -growth than that predicted by Eq. (64).

Not enough work has been done to determine if the dispersion relation is consistent with Eq. (72).

If these results can be verified experimentally, then it would be possible to either relax fabrication tolerance or implode high-gain targets with wavelengths lower than $3 \omega$ to $4 \omega . \ldots \ldots$

The so-called Richtmyer-Meshkov instability ${ }^{17}$ has been ignored in this analysis. This instability, which results in secular growth at all surfaces of density discontinuity, after the passage of a shock, is only of

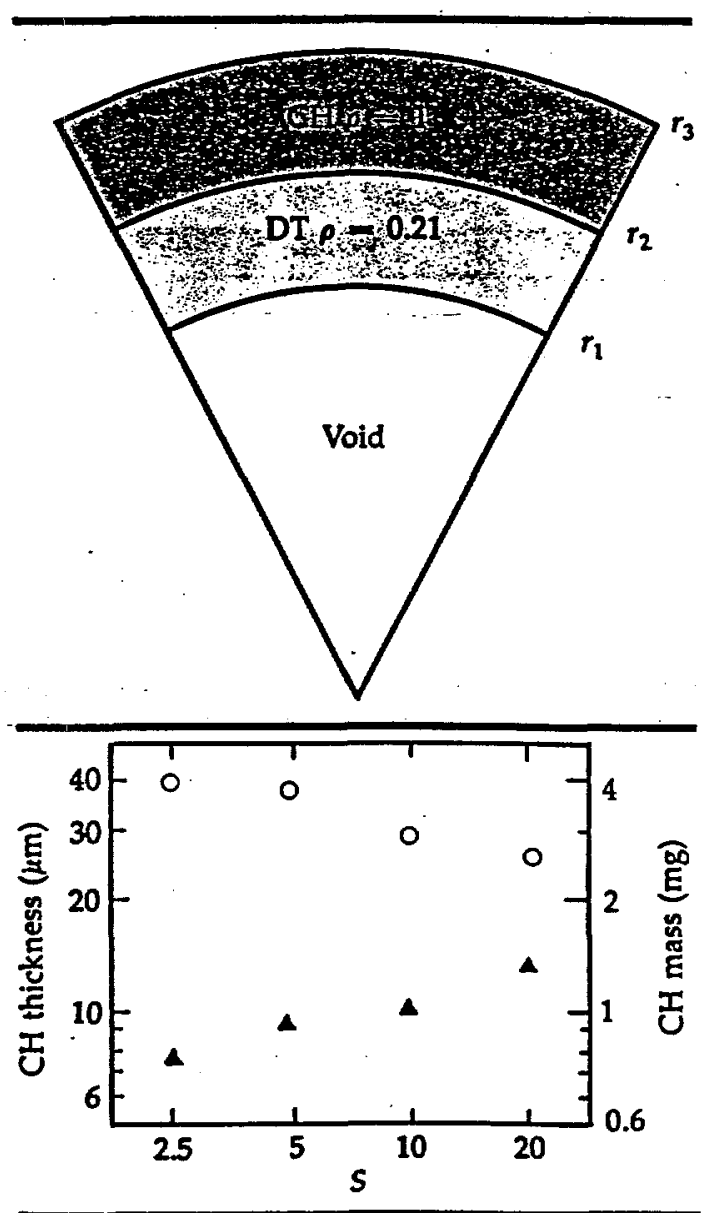

major importance for implosions that are driven by a few strong shocks. Since this growth can occur even at Rayleigh-Taylor stable surfaces, such as the pusher-fuel interface, it can dominate mix into the fuel strong-shock-implosions. However, for im plosions with nearly constant acceleration or a series of weak shocks, which is chara teristic of high-gain ICF targets, such surfaces are stable.

Author: J. D. Lindl 


\section{Plasma and LASNEX}

Laser-Plasma Coupling in LongScalelength, High- $Z$ Plasma

Introduction. Our experiments have demonstrated that short-wavelength laser light couples well with hohlraum targets. The $r$ maining task is to show that the coupling. remains excellent for the larger hohlraums' that will be used to drive high-gain targets These latter hohlraums will be irradiated i with megajoules of light in stepped pulses' having durations of tens of nanoseconds.? Under these conditions, the light will inter act with long-scalelength, underdense 
plasmas in which various instabilities could, in principle, become efficient. However, due to the strong collisionality of the high- $Z$ plasmas generated by the larger hohlraums, we expect the coupling to remain favorable, provided the laser-light intensity is limited to a modest value and filling is avoided.

We here examine some important features of the coupling in long-scalelength, high- $Z$ plasmas. Significant inverse bremsstrahlung absorption is predicted to occur at densities much less than the critical density $\left(n_{c}\right)$, a feature not commonly encountered in laser experiments to date. We consider the role of underdense-plasma instabilities, using LASNEX calculations to estimate the conditions in the wall plasma of a reactor hohlraum irradiated with $0.25-\mu \mathrm{m}$ laser light. For the nominal irrzediation and plasma conditions, we find that the various laser-driven plasma instabilities are either below or near the threshold. This result is very encouraging, especially since both experiments ${ }^{56}$ and calculations indicate that we can operate by a factor of 3 to 10 above the threshold intensity with tolerable coupling. Nova experiments will allow us to check the coupling and plasma conditions with scalelengths closer to those of reactor targets.

\section{Collisional Absorption in Very}

Underdense Plasmas. In long-scalelength, high-Z plasmas, short-wavelength laser light can be collisionally absorbed at densitics much less than the critical density. This important feature is shown by some estimates, below. The collisional damping rate, " of a light wave is

$p \approx \frac{10^{-10} \ln \Lambda n Z}{\theta_{\mathrm{keV}}^{3 / 2}}\left(\frac{n}{n_{\mathrm{cr}}}\right)$,

where $Z$ is the charge state, $\ln \Lambda$ is the Coulomb logarithm, $\theta_{\mathrm{keV}}$ is the electron temperature in $\mathrm{keV}, \boldsymbol{n}$ is the electron density, and $n_{\mathrm{cr}}$ is the critical density. Taking In $\Lambda \approx 5$, and noting that $n_{c \tau} \approx 10^{21} / \lambda_{\mu}^{2}$ (where $\lambda_{\mu}$ is the laser-light wavelength in microns), we obtain

$v \approx \frac{5 \times 10^{11} Z}{\lambda_{\mathrm{a}}^{2} \theta_{\mathrm{keV}}^{3 / 2}}\left(\frac{n}{n_{\mathrm{cr}}}\right)^{2}$

We next assume for simplicity an exponential density profile with scalelength $L$.
The requirement that the light be about half-absorbed by the time it reaches density $n$ is $\nu L / c \approx 1$, which becomes

$\frac{16.62 L\left(\frac{n}{n_{\mathrm{cr}}}\right)^{2}}{\lambda_{\mu}^{2} \theta_{\mathrm{keV}}^{3 / 2}} \approx 1$.

As an example, take $Z=50, \theta_{\mathrm{keV}}=3$, $L=0.05 \mathrm{~cm}$, and $\lambda_{\mu}=0.25$. Then, Eq. (83) gives $n / n_{\mathrm{cr}}=0.09$.(!) Thus, in longscalelength, high- $Z$ plasmas, significant collisional absorption takes place at densities much less than the critical density.

It is instructive to supplement Eq. (83) with an estimate that self-consistently relates the temperature to the incident intensity of the laser light. Using a freestreaming estimate of the heat flow, with electron flux limiter $f \approx 0.1$, we obtain

$\epsilon I \lambda_{\mu}^{2} \approx 2 \times 10^{13}\left(\frac{n}{n_{c r}}\right) \theta_{\mathrm{keV}}^{3 / 2}$,

where $I$ is the intensity and $\epsilon$ is the fractional absorption by density $n$. Substituting Eq. (84) into Eq. (83) gives

$\frac{3.3 \times 10^{14} \mathrm{ZL}\left(\frac{n}{n_{\mathrm{cr}}}\right)^{3}}{\epsilon I \lambda_{\mu}^{4}} \approx 1$.

As an example of the latter relationship, take $Z=50, \lambda_{\mu}=0.25, L=0.05 \mathrm{~cm}$, $\epsilon=0.5$, and $I \stackrel{\mu}{=} 5 \times 10^{14} \mathrm{~W} / \mathrm{cm}^{2}$. Then, $n / n_{\mathrm{cr}} \approx 0.1$. The self-consistent temperature estimated from Eq. (84) is then about $4 \mathrm{keV}$, which is rather high because sizable absorption is occurring at only a fraction of the critical density.

Instabilities in the Wall Plasma. Since inverse bremsstrahlung absorption is very efficient in long-scalelength, high- $Z$ plasmas, we expect excellent coupling if collective plasma effects are sufficiently suppressed. As discussed in previous Laser Annuals, ${ }^{57-59}$ underdense plasma instabilities can become efficient when laser light interacts with the very long-scalelength plasmas produced when a hohlraum fills with plasma, as demonstrated in many experiments with the Shiva laser. Even if a hohlraum is designed to avoid filling, long 
Table 2-6. Pulse shape for a reactor target.
Plasma and LASNEX

\begin{tabular}{ccc}
\hline Time (ns) & Power (W) \\
\hline $0-38.55$ & & $2.5 \times 10^{12}$ \\
$38.55-45.2$ & & $1.1 \times 10^{13}$ \\
$45.2-48.2$ & & $4 \times 10^{13}$ \\
$48.2-50$ & $1.5 \times 10^{14}$ \\
$50-55$ & & $2.4 \times 10^{14}$ \\
\hline & $L$ & $I$ \\
$Z$ & $(\mu \mathrm{m})$ & $\left(W / \mathrm{cm}^{2}\right)$ \\
\hline 35 & $<30$ & $\sim 0$ \\
47 & $<30$ & $\sim 1.2 \times 10^{14}$ \\
53 & $\sim 350$ & $\sim 1.5 \times 10^{14}$ \\
60 & $\sim 3400$ & $\sim-3 \times 10^{14}$ \\
\hline
\end{tabular}

Table 2-7. A summary of the LASNEXcalculated plasma conditions at the end of the pulse in a gold disk irradiated with $2 \mathrm{MJ}$ of $0.25-\mu \mathrm{m}$ light.

Fig. 2-64. A LASNEXcalculated density profile for a disk irradiated with a 2-M] shaped pulse of $0.25 \mu \mathrm{m}$ light.

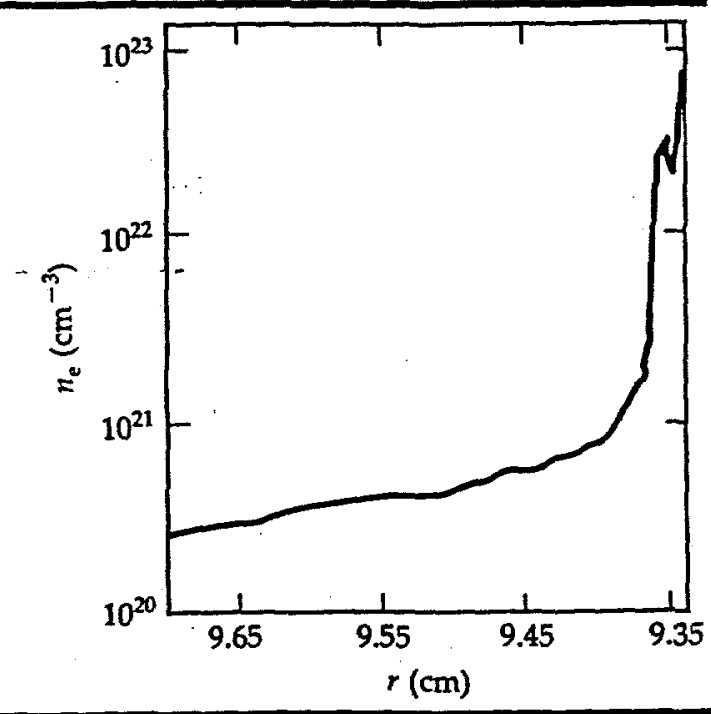

\begin{tabular}{lll}
\hline \multicolumn{1}{c}{ Instability } & $\begin{array}{c}\text { Gradient threshold } \\
\left(W / \mathrm{cm}^{2}\right)\end{array}$ & $\begin{array}{c}\text { Collisional threshold } \\
\left(\mathrm{W} / \mathrm{cm}^{2}\right)\end{array}$ \\
\hline $2 \omega_{\mathrm{pe}}$ & $8 \times 10^{14}$ & $1.3 \times 10^{16}$ \\
RS $\left(0.25 n_{\mathrm{cc}}\right)$ & $3 \times 10^{15}$ & $1.3 \times 10^{16}$ \\
RS $\left(0.1 n_{\mathrm{c}}\right)$ & $10^{14}$ & $10^{14}$ \\
RS $\left(0.025 n_{\mathrm{cr}}\right)$ & $10^{12}$ & $8 \times 10^{14}$ \\
BBS $\left(0.1 n_{\mathrm{c}}\right)$ & $2.5 \times 10^{15}$ & - \\
BBS $\left(0.025 n_{\mathrm{cd}}\right)$ & $1.8 \times 10^{15}$ & - \\
PE $\left(0.1 n_{\mathrm{cc}}\right)$ & $10^{15}$ & $6 \times 10^{14}$ \\
PF $\left(0.025 n_{\mathrm{cr}}\right)$ & $8 \times 10^{14}$ & $10^{14}$ \\
TF $\left(0.1 n_{\mathrm{cr}}\right)$ & $5 \times 10^{14}$ & $2 \times 10^{15}$ \\
TF $\left(0.025 n_{\mathrm{cr}}\right)$ & $3 \times 10^{15}$ & $2 \times 10^{15}$ \\
\hline
\end{tabular}

Table 2-8. Estimated threshold intensities of a number of instabilities for the plasma conditions shown in Table 2-7. scalelengths can still be encountered in the plasma blowing off the walls of a large hohiraum irradiated with long pulses of light.

To begin the assessment of conditions in the wall plasma of a reactor hohlraum, we have carried out some LASNEX calculations of gold disks irradiated with a 2-M] pulse of $0.25-\mu \mathrm{m}$ light. The pulse shape, shown in Table 2-6, concludes with a 5-ns step for which the nominal intensity at the wall is about $3 \times 10^{14} \mathrm{~W} / \mathrm{cm}^{2}$. Table $2-7$ shows the underdense plasma and irradiation conditions near the end of the final pulse, as calculated using an electron flux limiter of 0.1 and non-LTE $x$-ray physics in LASNEX.
Note that about half the light is absorbed by $0.1 n_{c}$ and that the plasma in the very, underdense region is rather hot $\left(\theta_{e} \sim 5 \mathrm{ke}\right.$

These results are in rough agreement with our estimates of inverse bremsstrah lung absorption given earlier in this article under "Collisional Absorption in Very Underdense Plasmas." When significant at. sorption occurs, the density scalelength be gins to be steepened by the localized deposition of energy. Figure 2-64 shows a density profile from the LASNEX calculas tion near the end of the pulse. The scalelength at $n=0.1 n_{c r}$ is $\approx 350 \mu \mathrm{m}$, which i approximately the classical absorption length at this density, rather than the muci larger distance that is a typical sound velo ity times an expansion time. Note the shâ. transition from hot, low-density plasma to dense, efficiently radiating-hence, coldplasma. In this transition layer, the electroi: pressure is approximately a constant. Sizable inverse bremsstrahlung absorption als occurs in this layer, although very little ligi reaches as high as the critical density.

For the plasma and irradiation condition discussed above, we find that the various laser-driven plasma instabilities are either below or near threshold, as shown in Tabl 2-8. For example, collisional thresholds are quite high $\left(\sim 10^{16} \mathrm{~W} / \mathrm{cm}^{2}\right)$ for instabilities $0.25 n_{\mathrm{cr}}$ Raman sidescatter near $0.1 n_{\mathrm{c}}$ is near threshold, but Raman scatter at much lower density is stabilized by strong Land: damping of the plasma waves. Brillouin sidescatter lies near threshold, but backsca ter is below threshold. Thermal filamenta tion is well below threshold, but might be more significant earlier in the pulse when the plasma is cooler. This assessment of th instabilities is encouraging, especially sinc calculations and experiments indicate that we can operate moderately above thresho without seriously degrading the coupling.

Conclusions. There are significant unce tainties in the modeling of the plasma con ditions. For example, LASNEX calculations with a lower electron flux limiter $f \approx 0.03$ yield nearly the same electron temperature in the low-density plasma $\left(n \approx 0.025 n_{c}\right)$, but the sharp transition layer extends dow; to about $0.05 n_{\mathrm{c}}$. In this case, the Raman it stability near $0.1 n_{\mathrm{cr}}$ would be stabilized by the density gradient. We have also estimated two-dimensional effects by onedimensional spherical LASNEX calculation: 
Ifrig a radius of twice the focal-spot diamand a flux limit of 0.1 . In this case, the than density jump extends down to about $005 n_{0}$ and the maximum electron temoferature drops to $3 \mathrm{keV}$. We are continuing 6 look at the complications caused by sizble intensity modulation in the laser Feams, oblique incidence of the light onto the hohlraum walls, and possible plasma incumulation due to filling. Nova experiments are being planned to check the plasma conditions and the coupling in plasmas with scalelengths closer to those in reactor targets.

Authors: W. L. Kruer, K. G. Estabrook, B. F. Lasinski, and W. C. Mead 


\section{Fusion Experiments}

\section{Contents}

Introduction

Suprathermal-Electron Scaling Experiments

Introduction

Filling Mode

Half-Caim Experiments

Conclusions

Radiation-Drive Scaling at $2 \omega$ and $4 \omega \ldots \ldots \ldots \ldots \ldots \ldots \ldots \ldots \ldots \ldots, \mathbf{4}, \mathbf{7}$

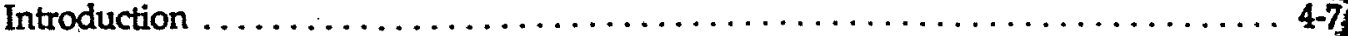

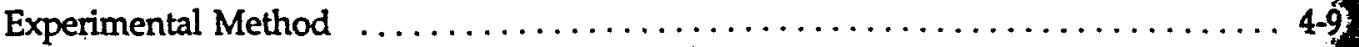

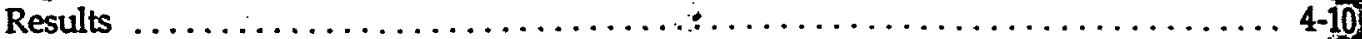

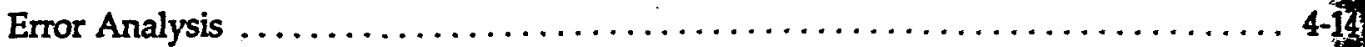

Aluminum Ablation $. \ldots \ldots \ldots \ldots \ldots \ldots \ldots \ldots \ldots \ldots \ldots \ldots \ldots \ldots \ldots, 4,15$

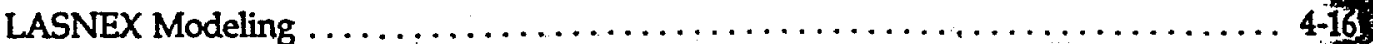

Preheat $\ldots \ldots \ldots \ldots \ldots \ldots \ldots \ldots \ldots \ldots \ldots \ldots \ldots \ldots \ldots \ldots \ldots \ldots \ldots \ldots, 4,18$

Primary/Secondary Drive Measurements on an 0.8-Scale Cairn . ............ 4-19

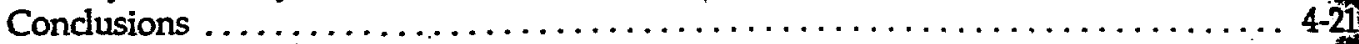

Results of the Novette Implosion Experiments $\ldots \ldots \ldots \ldots \ldots \ldots \ldots \ldots \ldots \ldots \ldots$ 4-21

Introduction $\ldots \ldots \ldots \ldots \ldots \ldots \ldots \ldots \ldots \ldots \ldots \ldots \ldots \ldots \ldots \ldots \ldots \ldots, 21$

Target Description and Irradiation Geometry $\ldots \ldots \ldots \ldots \ldots \ldots \ldots \ldots, \ldots \ldots, 4,22$

Novette Implosion Diagnostics $\ldots \ldots \ldots \ldots \ldots \ldots \ldots \ldots \ldots \ldots \ldots \ldots \ldots \ldots .4 .23$

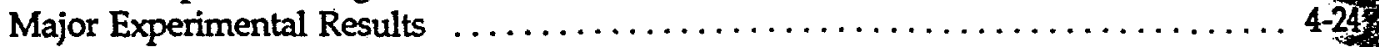

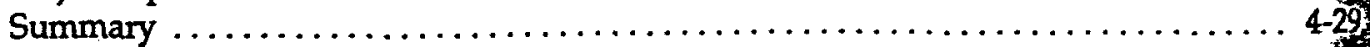

Analysis of the Novette Compression Series $\ldots \ldots \ldots \ldots \ldots \ldots \ldots \ldots \ldots \ldots \ldots$ 4-30

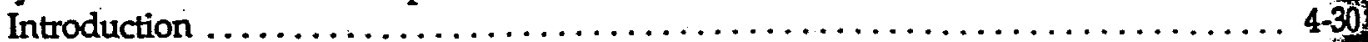

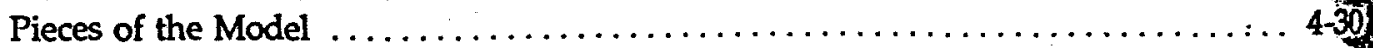

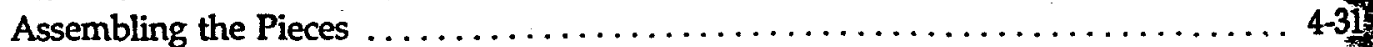

Checking our Model $\ldots \ldots \ldots \ldots \ldots \ldots \ldots \ldots \ldots \ldots \ldots \ldots \ldots \ldots \ldots \ldots \ldots \ldots \ldots, 32$

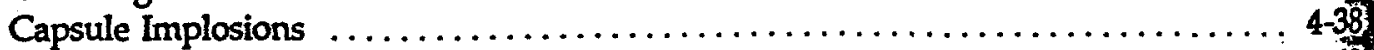

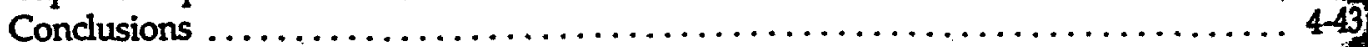

Analysis of Dante Data $\ldots \ldots \ldots \ldots \ldots \ldots \ldots \ldots \ldots \ldots \ldots \ldots \ldots \ldots \ldots \ldots, 4,43$

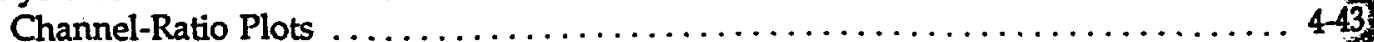

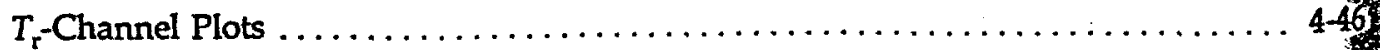

References $\ldots \ldots \ldots \ldots \ldots \ldots \ldots \ldots \ldots \ldots \ldots \ldots \ldots \ldots \ldots \ldots \ldots \ldots \ldots \ldots$ 4 4 q 


\section{Fusion Experiments}

\section{Introduction}

E. M. Campbell and J. D. Lindl

In this laser annual, we present the results of the highly successful hohlraum physics and implosion experiments conducted on the $0.53-\mu \mathrm{m}$ Novette facility. With up to $4 \mathrm{~kJ} /$ beam (1-ns FWHM) of energy available on target, we have been able to extend our hohlraum data base for both suprathermal electron production and radiation drive. In addition, the experiments were designed to provide an extensive comparison of measurements with the scaling models that have been developed for both suprathermal electrons (plasma filling) and radiation drive (Marshak wall loss) in laser-irradiated hohlraums.

In the first article in this section, "Suprathermal-Electron Scaling Experiments," we discuss suprathermal electron production in hohlraums irradiated with 1- and 3-ns pulses (FWHM) of 0.53- $\mu \mathrm{m}$ light. By changing both the laser energy and the hohlraum size, we varied the scaling parameter for the filling model $\left(A^{2} / \lambda^{2} E \tau\right.$, where $A$ is the hohlraum surface area, $\lambda$ is the laser wave length, $E$ is the laser energy, and $\tau$ is the pulse width) from 0.3 to $1.6 \times 10^{3}$. For the vast majority of the experiments, the high-energy $x$-ray bremsstrahlung yield indicated that the suprathermal electron levels were 3 to 10 less than the filling-model predictions. The experiments generally show that the interaction physics that results in suprathermal electrons in these hohlraums is dominated by the wall plasma and the first-bounce laser intensity.

The results of the radiation-drive experiments are discussed in the article, "Radiation-Drive Scaling at $2 \omega$ and $4 \omega$. " Using both absolute $x$-ray measurements and shock-wave measurements, we have examined Marshak scaling for a wide range of targets and laser conditions. This article also contains data from the $4 \omega$ series, in which we were able to generate $60-\mathrm{MBar}$ radiation-driven shocks in aluminum samples with negligible preheat $\left(f_{n}<10^{-3}\right)$. The $4 \omega$ experiments will be discussed more fully in next year's annual report.

The implosion experiments are discussed in the next three articles, "Results of the Novette Implosion Experiments," "Analysis of the Novette Compression Series," and "Analysis of Dante Data." These experiments examined the performance of radiation-driven capsules placed in primary-secondary hohlraums that were irradiated with both beams of Novette with up to $9 \mathrm{~kJ}(1 \mathrm{~ns})$ of $0.53-\mu \mathrm{m}$ light. The irradiated capsules were filled with 5 to $10 \mathrm{mg} / \mathrm{cm}^{3}$ of equimolar DT gas, and the performance of the capsules was measured using both the neutron yield and the pusher areal density integrated over the burn time. In addition to the implosion experiments, experiments were also conducted to characterize the hohlraum environment. Thin-walled hohlraums and burn-through foils were used to obtain information about the distribution of absorbed laser energy and about gradients in the $x$-ray drive, and multistepped foils (witness plates) and $x$-ray diodes were used to obtain information about the magnitude of the $x$-ray drive. These experiments determined that the effective radiation temperature in the hohlraum secondary was 130 to $170 \mathrm{eV}$, depending on the hohlraum size and laser energy, and that there were axial gradients in the radiation temperature. The observed hot electron fractions $\left(f_{\text {hot }}<10^{-2}\right)$ were so low that electron preheat was not an issue.

We observed a substantial improvement in the Novette gas-implosion results as compared with those obtained with the $1.06-\mu \mathrm{m}$ Shiva laser. While an increase in the pusher areal density of approximately $20 \times$ was seen for the 
high-density Shiva experiments conducted with single-shell targets, increases in areal density ranging from 40 to $90 \times$ have been measured at Novette. These large compressions, however, were not accompanied by an increase in the neutron yield. Neutron yields from $4 \times 10^{6}$ to $2 \times 10^{7}$, implying mass-averaged fuel temperatures of 500 to $600 \mathrm{eV}$, were measured; these yields were 10 to 100 times less than expected from one-dimensional preshot calculations. Early analyses of these experiments indicate that the low yields may be due to drive asymmetries in the hohlraum.

\section{Suprathermal-Electron-- Scaling Experiments}

Introduction

During recent years, a number of experiments on the Shiva and Argus lasers have explored suprathermal-electron scaling for enclosed (hohlraum) targets. These experiments are important because suprathermal electrons preheat the DT fuel and reduce target performance. Experimenters using $1.06-\mu \mathrm{m}$ irradiation from the Shiva laser on targets small enough to produce radiation temperatures above $150 \mathrm{eV}$ found that more than half of the incident energy was converted into hot electrons having temperatures exceeding $40 \mathrm{keV}^{1}$ These hot electrons significantly limited the extent to which Shiva could compress the fuel. The results from Shiva, including a series of long-pulse scaling experiments, ${ }^{2}$ were explained by a filling model, which is described later in this article.

The first experiments to test the benefits of short-wavelength irradiation of hohlraum targets were made with the Argus laser. ${ }^{3}$ Small targets were irradiated with up to $90 \mathrm{~J}$ of $1.06-\mu \mathrm{m}$ light, $220 \mathrm{~J}$ of $0.53-\mu \mathrm{m}$ light, and $35 \mathrm{~J}$ of $0.35-\mu \mathrm{m}$ light. The filling model correctly predicted the hot-electron fractions and temperatures observed for $1.06-\mu \mathrm{m}$ irradiation, but predicted far more hot electrons than were observed with 0.53 - and $0.35-\mu \mathrm{m}$ irradiation. Also for the shortwavelength shots, the observed $x$-ray spectra tended to show only one hot component, rather than the two that were predicted by the model. Similar results have recently been obtained by KMS Fusion using $0.53-\mu \mathrm{m}$ light at energies comparable to those used on Angus. However, KMS used very small targets in all of their experiments, and it remained uncertain whether experiments with more energy, larger entrance holes, and larger first-bounce laser spots would perform differently.

This article reports the results of suprathermal-electron scaling experiments in which hohlraum targets were irradiated with $1-$ and $3-n s$ pulses of $0.53-\mu \mathrm{m}$ (green) light from the Novette laser. We irradiated both half-Cairns and full-Cairns. The halfCairns, designed to be irradiated with only one beam of the laser, provide the most direct comparison with results from Argus and Shiva. We shot 16 half-Cairns of three different sizes at energies from less than $1 \mathrm{~kJ}$ to almost $4 \mathrm{~kJ}$. The full-Caim targets were similar to the implosion targets used on Novette, with scattering cones to absorb and scatter the incident laser light. In this article, we will discuss only the half-Caim data. Some of the data on absorption and scattering, $x$-ray production and transport, and hot-electron transport for the fullCairns are presented in other articles later in this section (see "Results of the Novette Implosion Experiments," "Analysis of the Novette Compression Series," and "Analysis of Dante Data").

\section{Filling Model}

The filling model predicts the magnitude of both a hot and a superhot component of hot electrons. The model assumes that the hot electrons are produced after the volume of the hohiraum fills to roughly $25 \%$ of the critical density of the laser light." For fixed laser energy, pulse length, and hohlraum size, the model predicts that fewer hot electrons will be produced when targets are irradiated at shorter wavelengths. This beneficial effect of shorter wavelengths 
arises because the critical density increases as the square of the laser frequency; thus, as frequency increases, the hohlraum must fill to a higher density to reach $25 \%$ of the critical density.

The model neglects two other processes that may also reduce the hot-electron production at shorter wavelengths. First, at the higher densities, the temperatures become lower and the plasma becomes more collisional, thus reducing the magnitude of, and increasing the threshold for, the plasma instabilities. Second, the threshold intensities for instability are increased because the oscillating velocity of the electrons in the plasma is reduced (at a given intensity).

Finally, two additional caveats apply to the model. First, the laser entrance hole might be what was filling, rather than the volume of the hohlraum. Experiments have not yet eliminated either possibility, and both effects would have the same scaling. Second, the plasmas near the hohlraum wall will also contribute to the production of hot electrons, even without filling. ${ }^{5}$

\section{Half-Cairn Experiments}

Targets. We chose the half-Cairn targets on the basis of the hohlraum filling model, LASNEX calculations, and other considerations. The targets are similar to the primary section of the primary-secondary hohlraums used for the implosion series. As Fig. 4-1 shows, the diameter of a Novette 1.0-scale target is $1.5 \mathrm{~mm}$; the other target dimensions (length, entrance hole, Dante hole size and location, and radius of comer) are scaled to the target diameter as shown in the figure. The laser was focused at several different locations to vary the intensity of irradiation on the first-bounce surface.

Many of the targets were made of tungsten, with the wall thickness chosen to equal the range of a $140-\mathrm{keV}$ hot electron $(1.8 \mu \mathrm{m})$. Thus, the target walls were thick enough that the Marshak wave would not break through during the laser pulse and thin enough that the target would not absorb a large fraction of the $10-\mathrm{keV} \times$ rays produced by the hot electrons. The targets were made of tungsten because Los Alamos had already developed a fluidized-bed,

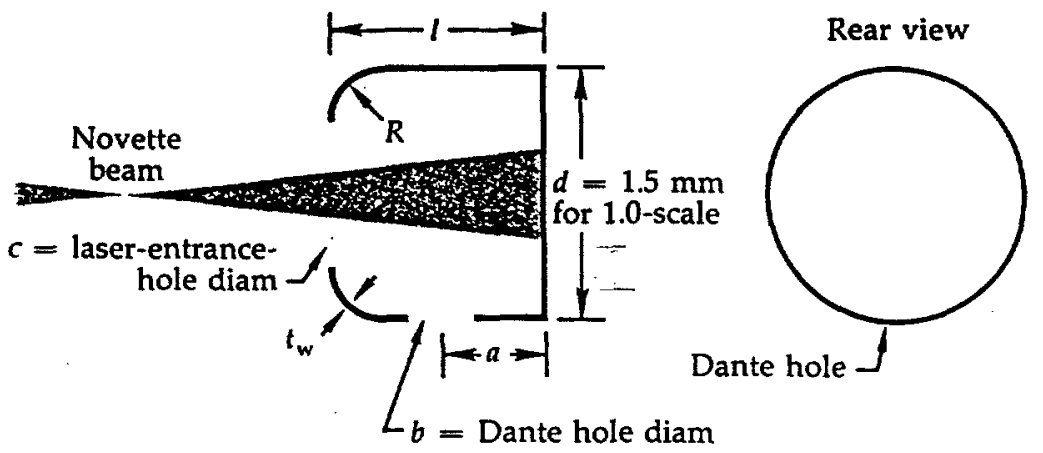

Dimensions

$$
\begin{aligned}
& d=\text { Outside diameter of cylinder }=1500 \mu \mathrm{m} \text { for } 1.0 \text {-scale } \\
& l=\text { Length of cylinder }=5 / 6 d \\
& a \quad=\text { Distance from back wall to center of Dante hole }=13 / 30 d \\
& b \quad=\text { Diameter of Dante hole }=4 / 15 d \\
& c=\text { Laser-entrance-hole diameter }=0.5 d \\
& t_{w}=\text { Wall thickness } \\
& R \quad=\text { Radius of front corner }=0.25 d \\
& g \quad=\text { Back corner angle }=90 \pm 2^{\circ}
\end{aligned}
$$

chemical-vapor-deposition process that produces high-quality, submicron-sized tungsten parts. We used some gold hohlraums with thick (roughly $15 \mu \mathrm{m}$ ) walls, either for comparison with the thin-walled tungsten hohlraums or to hold structures that the latter hohlraums could not accommodate. Flagand shield designs prevented the Dante and other diagnostics from measuring plume emissions near the laser entrance holes.

Diagnostics. We used $x$-ray images from the $8 \times$ microscope and images of the re- flected green light to verify that the laser beams successfully entered the target. In 3 of the 16 cases, the beam clipped the entrance hole; we had to estimate the fraction of the laser energy that entered the target before we could analyze these shots. The photodiode arrays, along with the incidentand reflected-energy measurements, determined the absorption, which varied from roughly $80 \%$ for 0.64 -scale targets to more than $90 \%$ for 1.0 -scale targets. We observed no indications of significant light scattering out of the hohlraum through any diagnostic holes. Table 4-1 summarizes the results of these shots. Shown in the table are the incident energy on target, average first-bounce intensity, target size, target area, and the filling parameter $A^{2} /\left(E \lambda^{2} \tau\right)$. Also shown in the table are the observed hot-electron
Fig. 4-1. Half-Cairn targets for Novette scaling experiments. 
Suprathermal-Electron Scaling Experiments

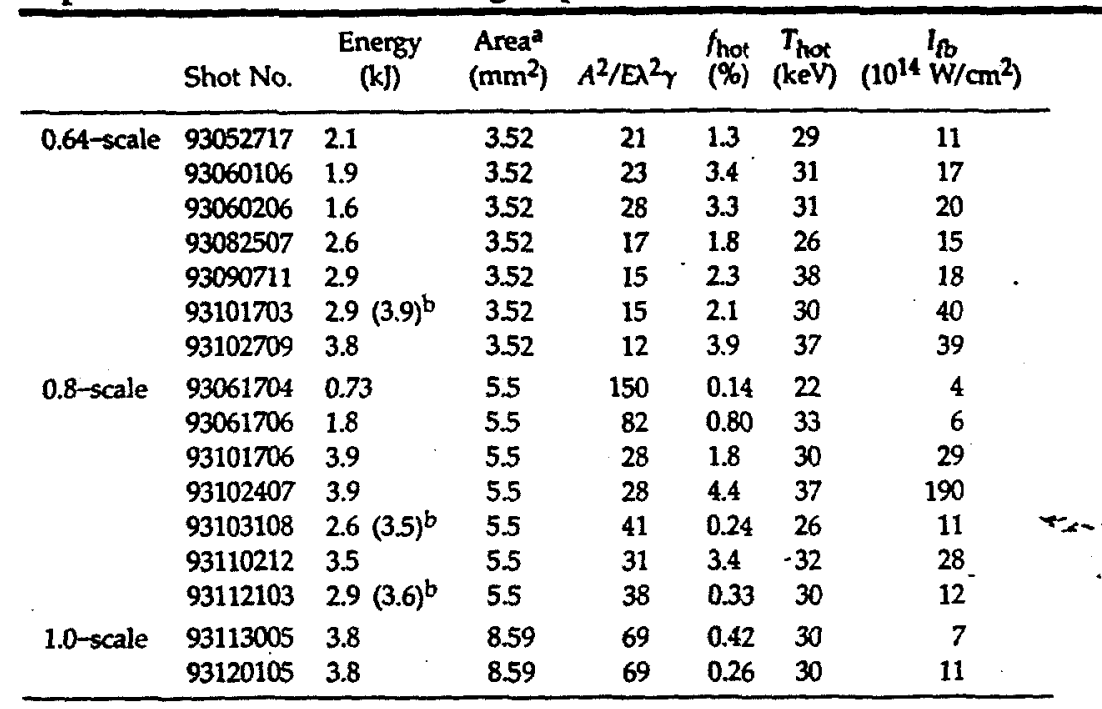

area includes rounding of comers, but does not compensate for laserentrance hole or diagnostic hole. Areas were calculated using standard dimensions; actual targets vary slightly.

b Laser beam clipped the entrance hole in three shots. Energy values shown are estimates of beam energy that entered the hohlraum; values in parentheses are nominal beam-energy levels.

Table 4-1. Half-Cairn experiments with 1-ns pulses.

Fig. 4-2. Measured hot-electron fractions shown as a function of average firstbounce intensity on the back wall of the target.

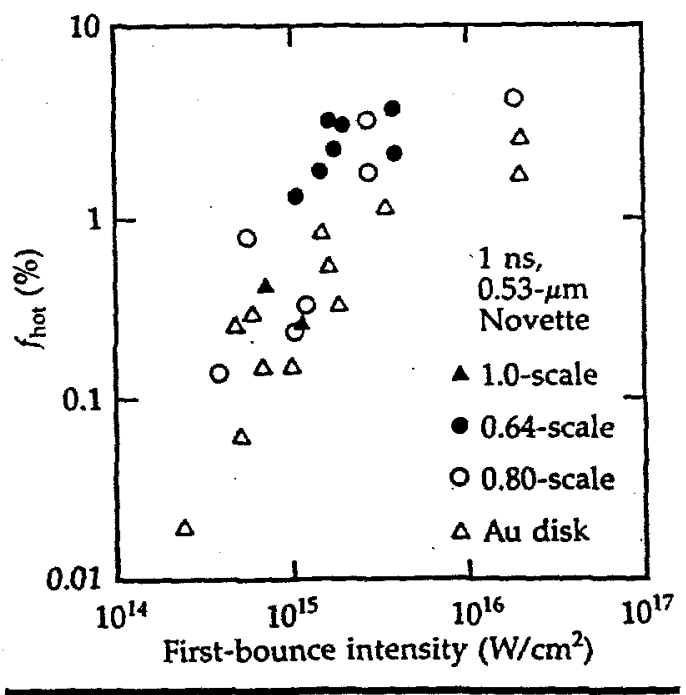

fraction and hot-electron temperature, $f_{\text {hot }}$ and $T_{\text {hot }}$ respectively.

Hot-Electron Fraction. Figure 4-2 shows the hot-electron fraction, inferred from the bremsstrahlung spectrum measured by the FFLEX diagnostic, as a function of average first-bounce intensity for 1-ns pulses. The figure shows data from 0.64-, 0.8-, and 1.0-scale half-Cairns, along with data from gold-disk targets. The hot-electron fractions from the hohlraums are at most 2 or 3 times larger than those from the disk targets. This small difference might be due to the greater electron-collection efficiency of the enclosed target, to production of hot electrons near the entrance hole of the half-Caim, or to some production of hot electrons throughout the volume of the half-Cairn. In contrast, hohlraums of similar size irradiated at similar energy with $1.06-\mu \mathrm{m}$ light on Shiva produced from 10 to 100 times more hot electrons than comparable disk targets fagain on the basis of bremsstrahlung measurements). The hotelectron yields inferred for disk targets on Shiva were comparable to those observed on Novette, but the hot-electron temperature for the Shiva disks was significantly higher.

Because of the similar hot-electron fractions observed from hohlraums and disk targets on Novette, our studies of disk targets reveal the most probable source of hot electrons in the hohlraums. Figure 4-3 shows the hot-electron fraction from gold disk targets as a function of the fraction of laserenergy observed as Raman-scattered light from densities below quarter-critical density. Based on this and other data, we concluded that Raman scattering may be a principal source of hot electrons in these disk-target experiments. This conclusion suggests that Raman scattering is a significant (and may be the dominant) source of hot electrons in the hohlraum targets irradiated on Novette as well.

Long-Pulse Experiments. Because we observed very few hot electrons when we used 1-ns pulses, we attempted some 3-ns experiments to allow more time for the hohlraum to fill. For the long-pulse experiments, we measured the yield of suprathermal electrons from 0.64 -scale halfCairns irradiated with $2 \mathrm{~kJ}$ in' 3 ns. The output pulse shape was roughly square during the 3-ns experiments, as opposed to the Gaussian pulses used for the 1-ns shots. We had hoped these 0.53- $\mu \mathrm{m}$ (green-light) measurements would provide a large $f_{\text {hot }}$ to compare with subsequent $0.27-\mu \mathrm{m}$ (ultraviolet) experiments.

The results of the 3-ns green-light shots are summarized in Table 4-2. These experiments were performed with best focus placed at the front face of the hohlraum, sc that pointing difficulties anticipated in the succeeding ultraviolet experiments would $b$ minimized. For the first two shots, the firstbounce intensity was about $2 \times 10^{15} \mathrm{~W} / \mathrm{cm}^{\text {: }}$ the intensity at the entrance hole was a fer times larger. Because the first two shots might have been just below threshold for stimulated Raman scattering, we increased the laser energy by nearly a factor of 2 on 
Suprathermal-Electron Scaling Experiments

the third shot. As shown in Table 4-2, the level of suprathermal electrons did increase, but $f_{\text {hot }}$ did not approach the value of $39 \%$ predicted by the filling model.

Comparison of Data with Predictions. An observed $x$-ray spectrum indicates the magnitude and energy of the hot electrons striking the target. Figure 4-4 shows the measured spectrum and the filling-model prediction for the highest energy 1-ns shot on a 0.64-scale half-Cairn; the observed spectrum shows a single temperature of about $37 \mathrm{keV}$. For most hohlraum targets, the temperature was near $30 \mathrm{keV}$, and the $x$-ray spectra from these targets are quite similar to the spectra from disk targets irradiated at comparable intensity. We never observed a superhot tail on the $x$-ray distributions from the Novette experiments, in agreement with Argus results at 0.53 and $0.35 \mu \mathrm{m}$, but in sharp contrast to Shiva results at $1.06 \mu \mathrm{m}$. The latter results suggest that some process was above threshold for the 1.06- $\mu \mathrm{m}$ experiments and below threshold (or produced a much cooler hot-electron spectrum) for the shortwavelength experiments.

As mentioned, the filling-model prediction differs from the observed data. The predicted hot component, corresponding to an $f_{\text {hot }}$ of $23 \%$ at a temperature of $27 \mathrm{keV}$, is several times larger than the observed spectrum, which implies an $f_{\text {hot }}$ of $4 \%$. In addition, the predicted superhot component is not present in the data.

The data described above indicate that we have not observed hot-electron production as a result of hohlraum filling in these Novette green-light experiments. Definitive evidence to support this indication is provided by the optical/x-ray (OX) streak camera. Figure 4-5 shows OX data obtained from an 0.8-scale half-Cairn that was predicted by the filling model to produce an $f_{\text {hot }}$ of $8 \%$ with a $T_{\text {hot }}$ of $23 \mathrm{keV}$. The observed $f_{\text {hot }}$ and $T_{\text {hot }}$ are $2 \%$ and $30 \mathrm{keV}$, respectively. The lowest trace in Fig. 4-5 shows the $1 \omega$ laser pulse, which was used as a fiducial for the $O X$ diagnostic. The $2 \omega$ pulse incident on the target had a similar pulse shape and duration.

The second trace from the bottom in Fig. 4-5 is the hot $x$-ray pulse; most of the signal in this channel is produced by $x$ rays near $30 \mathrm{keV}$ in energy. The $x$ rays are produced throughout the laser pulse and are most intense at the peak of the pulse. In

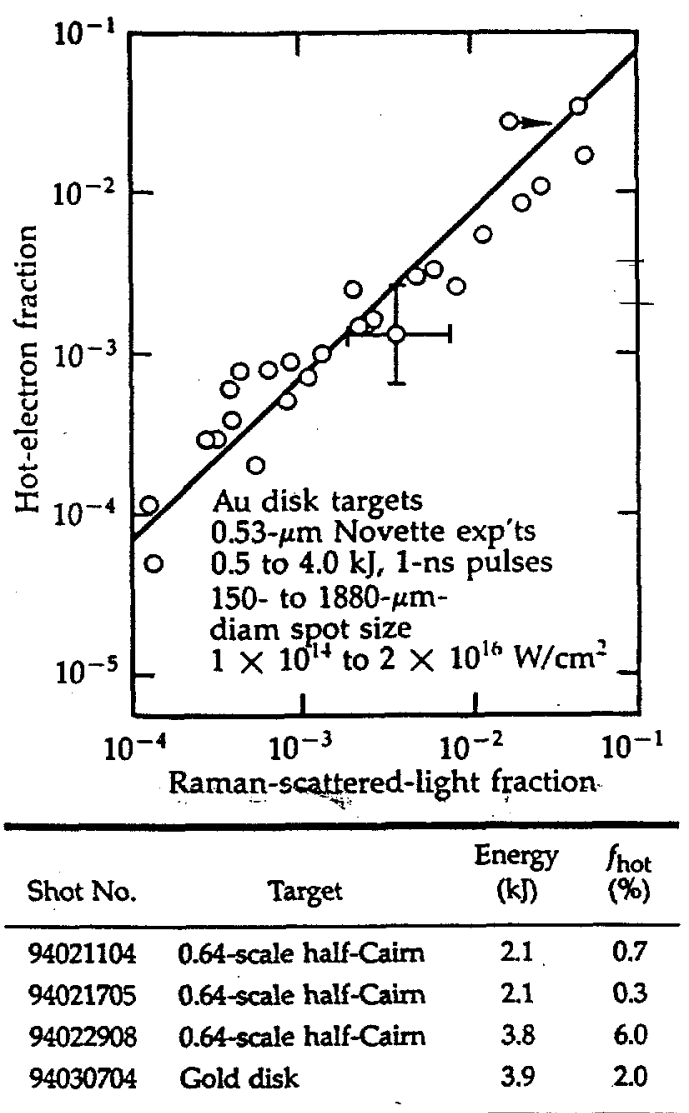

Fig. 4-3. Correlation ' of hot-electron fraction with Raman scattering.

Table 4-2. Half-Cairn and disk experiments with 3-ns pulses.

Fig. 4-4. Observed and predicted $x$-ray spectra from the FFLEX diagnostic. $\checkmark$

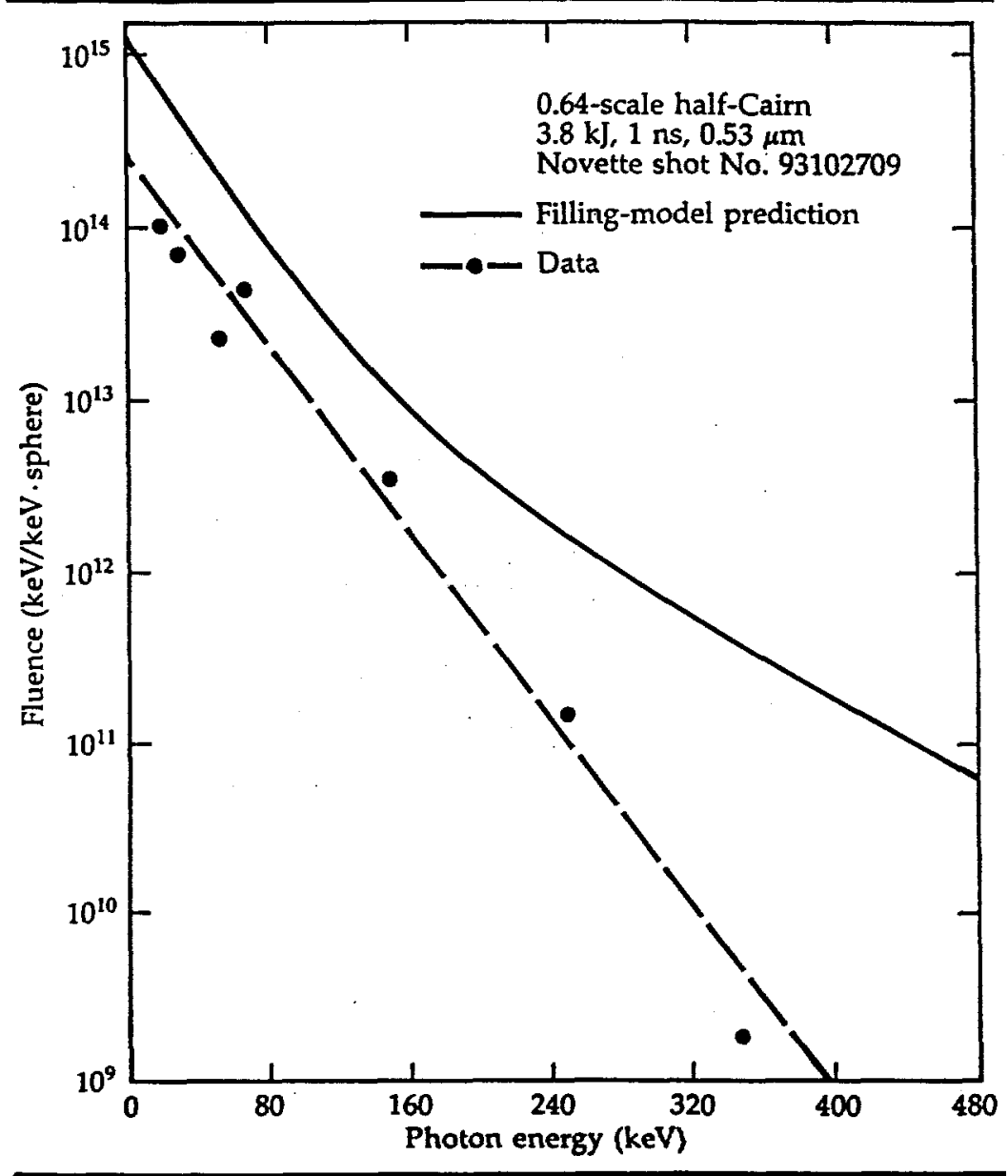


Fig. 4-5. Time history of $x$ rays and scattered light.
Suprathermal-Electron Scaling Experiments

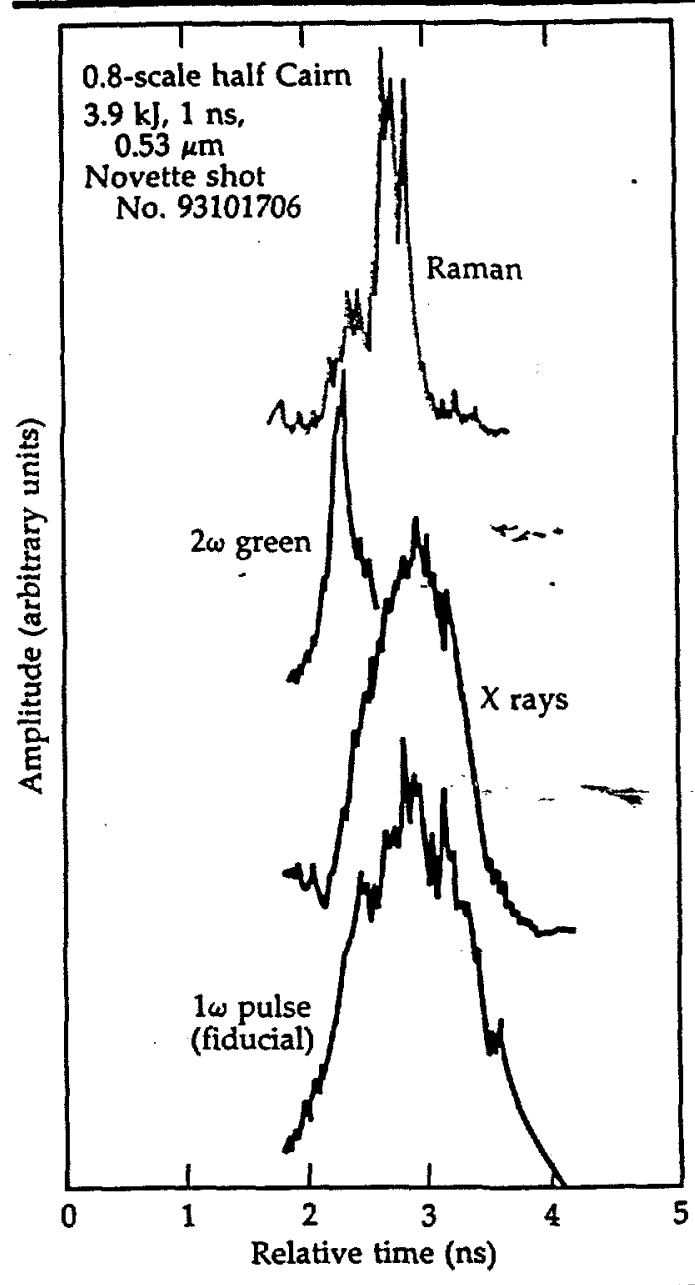

contrast, if hohlraum filling led to production of these $x$ rays, they would all be produced near the end of the pulse as predicted by the model. This latter behavior$x$-ray production near the end of the pulse-was observed during the long-pulse scaling experiments on Shiva. ${ }^{2}$ In agreement with the Novette half-Cairn data, $x$ rays are also produced throughout the pulse for gold-disk and exploding-gold-foil targets, further supporting the argument that the hot electrons producing these $x$ rays are produced near the hohlraum wall.

Figure 4-5 also shows the green light and Raman light scattered at $135^{\circ}$ to the incident laser direction. The scattering in this $135^{\circ}$ direction cannot come directly from the laser spot at the back of the hohlraum, so it may result from instabilities near the entrance hole or from reflections in the hohlraum. The green-light scattering decreases before the peak of the pulse, possibly due to inverse-bremsstrahlung absorption in the plasma. The Raman light, not very intense in this direction, is harder to interpret. Intense Raman scattering was detected in the backscatter direction, and the Raman scattering was imaged in the near-backscatter direction.

Figure 4-6 shows the filling-model prediction along with data from Shiva, Argus, and Novette. The Novette half-Cairns produced several times fewer hot electrons than predicted. In particular, the largest hot-electron fraction predicted for the 1-ns experiments was $23 \%$, and the observed hot-electron fractions were less than $5 \%$. Because of pointing, focusing, and pulse-length limitations in the Novette system, we were unable to perform experiments that were predicted to produce even higher hotelectron yields. The Novette hohlraums produced very few hot electrons, even in the 3-ns experiments where the hohlraums almost certainly filled with plasma. Experiments with exploding-foil targets should be : compared with these Novette results because the former experiments also produced $z$. very large plasmas and Raman yields on the order of $10 \%$, which should correspond : to hot-electron yields on the order of $5 \%$.

We conclude that the filling model does not accurately predict the scaling of hotelectron production with wavelength. There are several possible explanations for this behavior of the model, even without considering the role of effects at the laser entrance hole. First, Shiva directly irradiated a larger fraction of the hohlraum wall than did Novette because Shiva used 10 beams, while Novette used just 1 beam; this larger irradiation area might have caused the hohlraums to fill faster on Shiva, independently of the difference in wavelength. Second, the shorter wavelength of Novette caused higher absorption on the first bounce in the Novette targets, reducing the amount of light available to drive rapid plasma expansion from the sides of the target. Third, collisions and reduced electronoscillating velocities may reduce the hot-electron yields, either by saturating the instabilities or by increasing the instability thresholds. Recent simulations indicate that collisions can significantly reduce the hotelectron production caused by Raman backscatter. ${ }^{6}$ Conditions within the Novette hohlraum targets probably encourage collisional damping; e.g., for an electron temperature of $2 \mathrm{keV}$ and an intensity of $2 \times 10^{15} \mathrm{~W} / \mathrm{cm}^{2}$, the electron-ion collision 
Radiation-Drive Scaling at $2 \omega$ and $4 \omega$

frequency is comparable to the maximum growth rate of the Raman and twoplasmon-decay instabilities.

\section{Conclusions}

Both 1- and 3-ns experiments on Novette produced fewer hot electrons than were predicted by the filling model. In addition, the hot-electron fraction produced by hohlraum targets on Novette was shown not only to be quite sensitive to the laser intensity at the first-bounce surface but also to be comparable to the hot-electron fraction produced by disk targets irradiated at the same intensity. This latter finding - the comparability of hot-electron fractions for both hohlraum and disk targets-had not been observed previously and may be $o c-$ curring because we have so dramatically reduced the total hot-electron production in hohlraum targets. For comparison, Shiva hohlraum targets generally produced up to 100 times the hot-electron fraction produced by comparable Shiva disk targets.

Targets irradiated with $0.53-\mu \mathrm{m}$ light on Novette produced more drive and less preheat than comparable targets irradiated with 1.06- $\mu \mathrm{m}$ light on Shiva. The Novette data are similar to the Argus data in that only one hot-electron temperature was detected. Also, hot electrons were produced throughout the laser pulse, rather than at the end of the pulse as predicted by the filling model. This continuous production of hot electrons is definitive evidence that hohlraum filling is not the source of hot electrons observed on Novette.

The results of these Novette greenlight experiments suggest that hot-electron preheat may not be a major difficulty in conducting experiments on Nova. We are now investigating, through more detailed model calculations, the favorable suprathermal scaling found with the green-light experiments. There are grounds for caution when we project these results to Nova, however. We have not yet done experiments with picket-fence pulses, which allow time for plasma to develop between relatively high-intensity spikes. In addition, the Novette experiments did not always produce such small hot-electron yields. One full-Cairn produced a large hot-electron fraction in one primary, and one half-Cairn

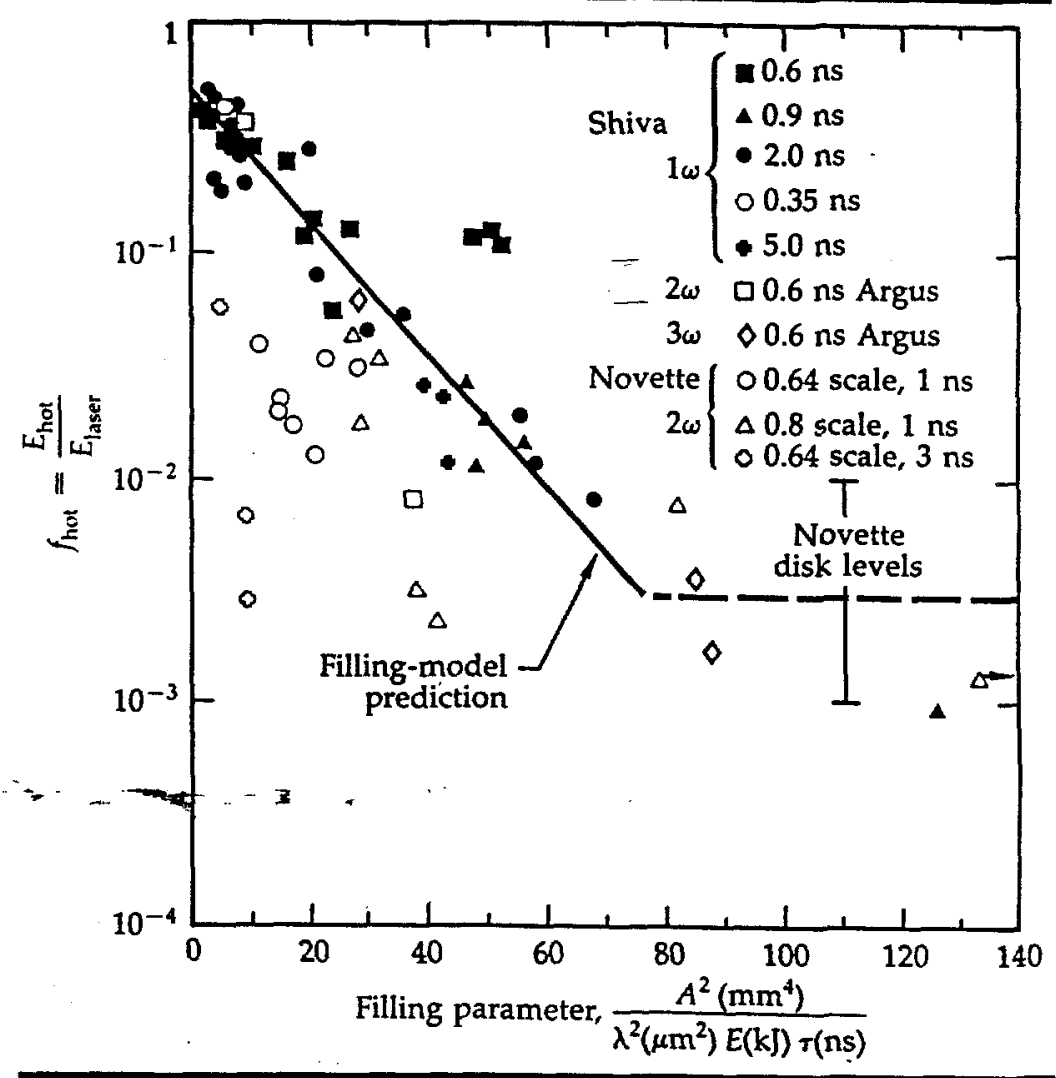

produced a hot-electron fraction of about $20 \%$. In addition, during preparations for the 3-ns experiments, a gold-disk target irFig. 4-6. Observed and predicted hotelectron fractions. radiated with an unknown pulse produced a hot-electron fraction of about $20 \%$. These exceptional cases show that, under conditions we have not yet fully identified, large hot-electron yields can be produced during $0.53-\mu \mathrm{m}$ experiments.

Authors: R. P. Drake and R. E. Turner

Major Contributors: B. F. Lasinski, D. W. Phillion, E. M. Campbell, W. C. Mead, and W. L. Kruer

\section{Radiation-Drive Scaling at $2 \omega$ and $4 \omega$}

\section{Introduction}

High-performance capsules designed for Nova require radiation drives in excess of $200 \mathrm{eV}$, with less than $1 \%$ of the laser energy in suprathermal electrons. These designs, with this low preheat, require $0.35-\mu \mathrm{m}$ light to obtain the required drive. Capsule design parameters are based on 
semi-empirical scaling laws derived from hohlraum data from Shiva using 1.06- $\mu \mathrm{m}$ light and from Argus using shorter wavelengths. (The Argus data are limited to low-drive conditions because of the limited energy available.) We have done a number of experiments on Novette to test these scaling laws at short wavelengths and high energy.

From our drive-scaling experiments, we have found that short wavelengths couple more efficiently to the hohlraums than 1.06- $\mu \mathrm{m}$ light. With a simple hohlraum at Novette, we have produced a drivè equiva: lent to $210 \pm 10 \mathrm{eV}$, with only $0.08 \%$ preheat using a $1.63-\mathrm{kJ}, 1-\mathrm{ns}$ pulse of $2630-\AA$ light. This drive was measured by the velocity of an ablatively driven shock in an aluminum target called a wimess plate. Although this target does not have sufficient symmetry to produce high-quality implosions, it does indicate that adequate drive conditions likely can be obtained on Nova with 10 beams of $0.35-\mu \mathrm{m}$ light.

Radiation-drive scaling is determined by partitioning the laser energy between the walls of the radiation case, the radiation field, and the low-density coronal plasma. The radiation drive is expected to be greater at short wavelengths, since less energy is scattered out of the hohlraum by the Brillouin and Raman instabilities and less energy goes into the hot corona.

For ICF hohlraums, most of the energy resides in the walls, allowing the radiation drive to be described by a Marshak wavescaling model. ${ }^{7}$ For supersonic Marshak waves, we can assume that the density is constant and write down the well-known radiation transport equation:

$\rho \frac{\partial \epsilon}{\partial t}=\frac{\partial}{\partial x} \frac{c}{3 \rho \kappa_{\mathrm{R}}} \frac{\partial}{\partial x} a T^{4}$

where $\rho$ is the density, $\epsilon$ is the specific internal energy density, $c$ is light speed, $\kappa_{R}$ is the specific Rosseland mean opacity, $T$ is the temperature, and $a T^{4}$ is the radiation energy density. This equation can be transformed into an ordinary differential equation by defining the scaling parameter $\xi=x / \sqrt{t}$. If both $\epsilon$ and $\kappa_{R}$ have power-law dependencies on the density and temperature, then scaling relations can be found for the energy in the wall and the penetration depth of the Marshak wave.
For instance, assume that the specific heat capacity, $c_{w}$ is constant and that the Rosseland mean free path, $l_{R}=1 /\left(\rho k_{R}\right)$, has - the dependence $l_{R}=l_{0 R} T^{\prime \prime} / \rho^{m+1}$. Then,

$E_{\mathrm{w}} \propto \sqrt{c_{\mathrm{v}} l_{\mathrm{OR}}} \rho^{-n+2} T^{\frac{\lambda+5}{2}} A_{\mathrm{w}} \sqrt{\tau}$

where $E_{\mathrm{w}}$ is the wall energy, $A_{\mathrm{w}}$ is the wall area, and $\tau$ is the time allowed for radiation diffusion.

The Marshak wave is subsonic in our experiments. Agreement with the fixeddensity idealization would be expected if neither the specific heat nor the specific opacity depended upon the density and if the mechanical energy of the hydrodynamic expansion were small. Rosen ${ }^{8}$ and Munro 9 have studied the subsonic Marshak wave and derived more complicated scaling relations. Rosen ${ }^{10}$ has obtained for gold

$\eta E_{L}=0.44 T_{\mathrm{R}}^{3.05} A_{\mathrm{w}} \tau^{0.57}+1.03 A_{\mathrm{h}} T_{\mathrm{R}}^{4} \tau$.

Areas are in $\mathrm{mm}^{2}, \tau$ is in $\mathrm{ns}, T_{R}$ is in heV $(100 \mathrm{eV})$, and $E_{\mathrm{L}}$ is in $\mathrm{hJ}(100 \mathrm{~J})$. The last term represents losses through holes of total area $A_{\mathrm{h}}$. In these units, $\sigma \equiv a c / 4=1.03$. The constant $\eta$ is the $x$-ray conversion efficiency for the laser light multiplied by the fraction of the light absorbed by the hohlraum. The EOS22 tables have been used for the gold opacity and equation of state.

We have determined the radiation drive both by using Dante to measure the absolute spectrum of the soft $x$ rays escaping through a diagnostic hole and by using an aluminum witness plate to measure the velocity of the shock driven by ablation pressure. The witness plate is a new drive diagnostic that we have developed at Novette. By using two separate techniques for measuring drive, we independently corroborated the applicability of the Marshak scaling model.

Our shock-velocity measurements indicate substantially higher radiation drives than are measured by Dante. If the radiation field were actually isotropic and uniform, the two measurements should have agreed. Dante measures the $x$-ray flux in one particular direction from one particular area, whereas the witness plate sees radiation from all parts of the hohlraum.

Three effects can contribute to the difference in the drive measurements. The first 
Radiation-Drive Scaling at $2 \omega$ and $4 \omega$

effect is the albedo. The Dante views a gold wall that is not directly irradiated by the laser. LASNEX computations for these experiments show that, at the peak of the laser pulse, the wall will reradiate at a temperature 5 to $10 \mathrm{eV}$ lower than the temperature based upon the incident radiation flux. This difference becomes quite small by the end of the laser pulse and is not nearly large enough to explain the discrepancy between the Dante and the shock-velocity measurements. The second effect is the angular distribution of the $x$ rays. Dante assumes a Lambertian $\cos \theta$ distribution, but, if the distribution were more nearly isotropic, the temperature inferred from the Dante measurement would be low. Finally, the third effect is hole closure, which. will also cause the Dante measurement to be low.

The shock velocity can be accurately measured only if the preheat is small. Excessive preheat $(>1 \mathrm{eV})$ of the back surface of the witness plate causes plasma blowoff, which changes the effective step height. In all of the $4 \omega(0.27 \mu \mathrm{m})$ experiments, and in most of the $2 \omega(0.53 \mu \mathrm{m})$ experiments, the preheat was small. If the back surface is preheated to temperatures greater than 1 to $1.5 \mathrm{eV}$, the thermal emission is observable by our instrument.

This article presents the radiation-drive measurements made at Novette for 1 ns and for both 0.53- and 0.27- $\mu \mathrm{m}$ laser wavelengths ( $2 \omega$ and $4 \omega$, respectively). At $0.53 \mu \mathrm{m}$, high electron preheat was sometimes observed for 0.64 -scale half-Caims irradiated with $3.5 \mathrm{~kJ}$. The FFLEX and witness-plate measurements of the preheat have been compared, and they agree, if it is assumed both that the electron preheat was uniform over the entire surface area and that electrons diffusing through the aluminum witness plate returned because of a self-consistent electric field.

\section{Experimental Method}

The shock velocity was measured by using the instrumentation shown in Fig. 4-7 to image and time-resolve the thermal ultraviolet light that was emitted after the shock breakouts. The aluminum witness plate had two steps differing in thickness by between 18 and $36 \mu \mathrm{m}$; the thinner step was between 30 and $43 \mu \mathrm{m}$ thick. The witness plate was bent around the outside of the hohlraum and patched over a hole that was $250 \mu \mathrm{m}$ in diameter for the smallest 0.33 -scale $4 \omega$ halfCairn targets and $300 \mu \mathrm{m}$ in diameter for all but one of the 0.50 - and 0.64 -scale $4 \omega$ half-Cairn targets. For all the $2 \omega$ Cairn targets and the 0.8 -scale $4 \omega$ half-Caim target, the hole diameter was $400 \mu \mathrm{m}$. The Dante hole was always the same size as, and was diagonally opposite to, the hole for the witness plate The holes were offset $30^{\circ}$, so

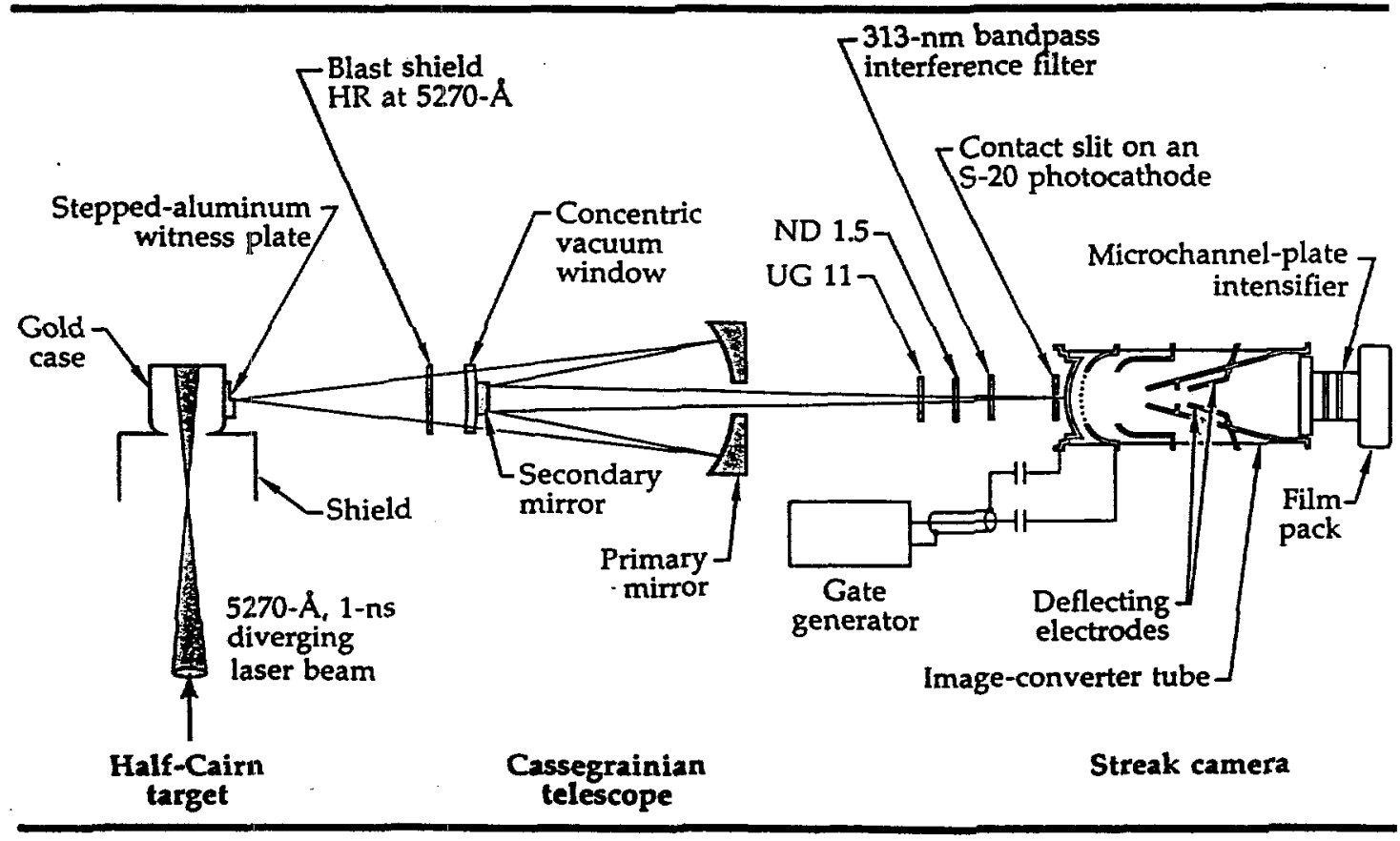

Fig. 4-7. Cassegrainian telescope and gated S-20 streak camera used to make the shock-velocity measurements. 
Fig. 4-8. The 200-eV hohlraum experiment. (a) Contour plot of the streak record. (b) Lineouts showing the shock breakouts.

\section{Radiation-Drive Scaling at $2 \omega$ and $4 \omega$}

the Dante saw only the gold wall and no aluminum. The witness-plate step was on the outside and there was a dividing barrier several hundred microns high between the steps.

Alignment was done just before each shot by putting a 200 - to $400-\mu \mathrm{m}$-diam microballoon in the witness-plate position. The microballoon was then backilluminated with white light from a largediameter fiber-optic bundle placed $1 \mathrm{~cm}$ back. Magnifications were 9.6 for the telescope and 1.09 for the streak camera. The streak camera had a 500- $\mu \mathrm{m}$-wide contact slit, an S-20 streak tube with a sapphire window, and an external krytron gate generator to gate off the streak tube about 20 ns after the end of the sweep. The streak cantera had been absolutely calibrated using the Monojoule laser, and the transmission or reflection curves for all the mirrors, windows, and filters had been measured. Thus, we were able to measure the absolute brightness of the shock, which is directly related to the shock temperature, although the observed temperature may be different than the temperature behind the shock in the dense material.

\section{Results}

The highest shock velocity of $5.75 \times 10^{6}$ $\mathrm{cm} / \mathrm{s}$ was measured for shot No. 94042604, which was a 0.50 -scale half-Caim irradiated with $1.63 \mathrm{~kJ}$ of $2630 \AA$ light in $1 \mathrm{~ns}$. The aluminum steps were nominally 46 and $70 \mu \mathrm{m}$ thick, and the step height was measured to be $24.1 \pm 1 \mu \mathrm{m}$. A contour plot of the streak record is shown in Fig. 4-8(a). The shocks break out at 370 ps for the $46-\mu \mathrm{m}$ step [(Fig. 4-8(b)] and at 790 ps for the 70- $\mu \mathrm{m}$ step [Fig. 4-8(c)]. The breakout times are measured from the peak of the laser pulse to the $50 \%$ points. Rise times for both shocks are about 70 ps. (Rise time is defined by first drawing the tangent to the shock rise at the $50 \%$ point, then obtaining the

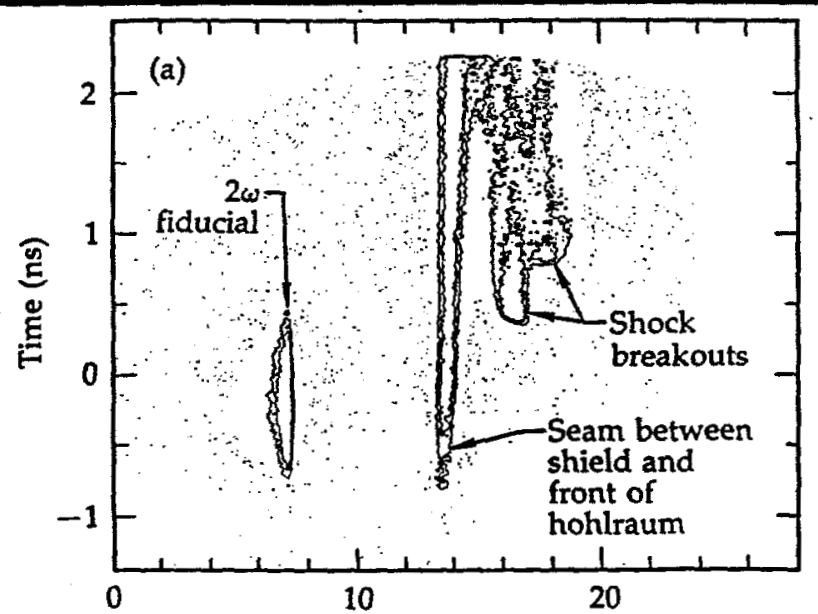

Position along width of the microchannel plate (mm)
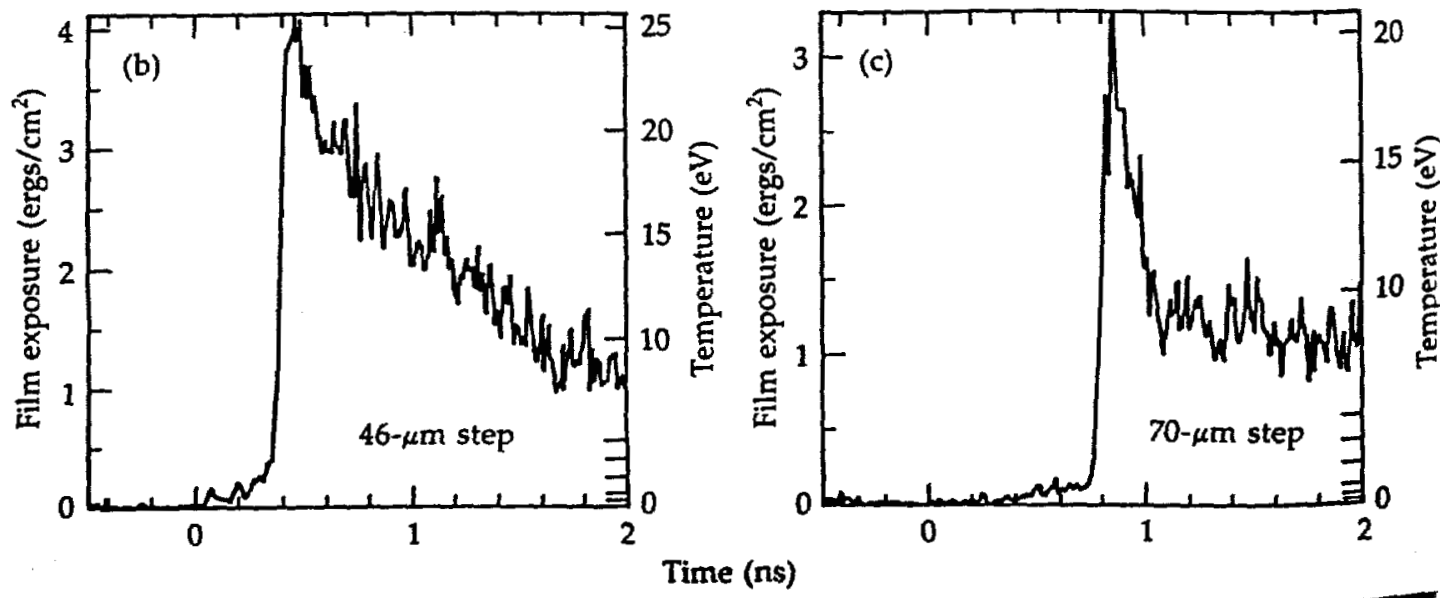
times corresponding to the 0 and $100 \%$ crossings for this tangent. The difference is the rise time.)

Shock brightness was fairly uniform over the steps, except near the edges. The magnification to the film (or the microchannel plate) was $10.4 \pm 0.5$, so that the image of each $150-\mu \mathrm{m}$-wide step should be $1.56-\mathrm{mm}$ wide. The flash has a temperature of $23 \mathrm{eV}$ for the first shock and $17 \mathrm{eV}$ for the second shock. A shock velocity of $5.75 \times 10^{6} \mathrm{~cm} / \mathrm{s}$ corresponds to an ablation pressure of $66 \mathrm{Mbar}$ and a radiation temperature of $210 \mathrm{eV}$. The theoretical temperature behind the shock in the dense material is $27 \mathrm{eV}$. The bright vertical line to the left of the shock signals in the streak record comes from the seam between a shield and the front of the Cairn. The shield prevented the Cassegrainian telescope from seeing the plume from the laser entrance hole and also much reduced the heating of the back of the witness plate by suprathermal electrons going around the outside of the Cairn.

Dante measured a radiation temperature of $175 \mathrm{eV}$ through a $400-\mu \mathrm{m}$-diam diagnostic hole with a line of sight at $30^{\circ}$ to the normal. For this experiment, then, the shock-velocity measurement gave a radiation temperature $35 \mathrm{eV}$ higher than Dante. For most of the $4 \omega$ experiments, Dante was about $30 \mathrm{eV}$ lower than the shock-velocity measurements; for the $2 \omega$ experiments; Dante was usually about $17 \mathrm{eV}$ lower.-

The $2 \omega$ and $4 \omega$ experiments are summarized in Tables 4-3 and 4-4, respectively. In deriving a radiation temperature, $T_{R}$, and an ablation pressure, $P_{a b l}$, from the measured shock velocity $u_{\mathrm{s}}$, we have used the strongshock scaling relations $P_{\mathrm{abl}} \propto u_{\mathrm{s}}^{2}$ and $T_{\mathrm{R}} \propto u_{\mathrm{s}}^{4 / 7}$. The point at $u_{\mathrm{s}}=5 \times 10^{6} \mathrm{~cm} / \mathrm{s}$, $P_{\mathrm{abl}}=50 \mathrm{Mbar}$, and $T_{\mathrm{R}}=195 \mathrm{eV}$ is used to determine the constants of proportionality. The aluminum Hugoniot curves were generated using the $\mathrm{H}$-Division code HYBRID and the equation-of-state table EOS811. For the radiative ablation of aluminum, LASNEX was used to connect the radiation temperature to shock velocity and shock temperature [Fig. 4-9(a)] and to shock pressure [Fig. 4-9(b)]. Standard x-ray drives from gold hohlraums irradiated by 1-ns laser pulses were used by LASNEX to calculate the aluminum ablation. The radiation

\begin{tabular}{|c|c|c|c|c|c|c|c|c|c|c|}
\hline Shot No. & Scale & $\begin{array}{c}E_{\mathrm{L}} \\
(\mathrm{kJ})\end{array}$ & $\underset{\left(\mathrm{mm}^{2}\right)}{A_{\mathrm{h}_{\mathrm{h}}}}$ & $\underset{\left(\mathrm{mm}^{2}\right)}{\left.A_{w}\right)}$ & $\begin{array}{l}T_{\text {hot }} \\
\text { (keV) }\end{array}$ & $\begin{array}{l}f_{\text {hot }} \\
\text { (\%) }\end{array}$ & $\begin{array}{c}\text { Shock velocity } \\
\begin{array}{c}u_{\mathrm{s}} \\
(\mathrm{cm} / \mathrm{s})\end{array}\end{array}$ & $\begin{array}{c}p_{\mathrm{abl}} \\
\text { (Mbar) }\end{array}$ & $\begin{array}{c}T_{\mathrm{R}} \\
(\mathrm{eV})\end{array}$ & $\begin{array}{c}\text { Dante } \\
T_{\mathrm{R}} \\
(\mathrm{eV})\end{array}$ \\
\hline 93120105 & 1.0 & 3.81 & 0.724 & 8.138 & 30 & 0.3 & - & - & - & 150 \\
\hline $94010905^{a}$ & 0.64 & 1.64 & 0.490 & 3.276 & 30 & 3.1 & $4.56 \times 10^{6}$ & 42 & 185 & 168 \\
\hline $94011009^{a}$ & 1.0 & 3.74 & 0.730 & 8.692 & 31 & 1.0 & $3.63 \times 10^{6}$ & 26 & 162 & 156 \\
\hline $94020211^{a, b}$ & 0.64 & 4.22 & 0.600 & 3.450 & 33 & 5.4 & - & - & - & 189 \\
\hline $94020309^{a}$ & 0.64 & 1.53 & 0.440 & 3.399 & - & - & $4.88 \times 10^{6}$ & 48 & 193 & - \\
\hline 94020808 & 1.0 & 1.95 & 0.692 & 8.895 & 22 & $<0.6$ & $3.33 \times 10^{6}$ & 21 & 152 & 135 \\
\hline 94020905 & 0.64 & 1.14 & 0.525 & 3.302 & 29 & 2.6 & $3.75 \times 10^{6}$ & 28 & 165 & 149 \\
\hline $94020909^{b}$ & 0.64 & 3.41 & 0.513 & 3.184 & 31 & 18 & - & - & - & 173 \\
\hline
\end{tabular}

2Streak camera was out of focus for the shock-velocity measurement.

bHigh preheat made the shock-velocity measurement wrong.

\begin{tabular}{|c|c|c|c|c|c|c|c|c|c|c|}
\hline Shot No. & Scale & $\begin{array}{c}E_{\mathrm{L}} \\
(\mathrm{kJ})\end{array}$ & $\underset{\left(\mathrm{mm}^{2}\right)}{A_{\mathrm{h}}}$ & $\underset{\left(\mathrm{mm}^{2}\right)}{A_{\mathrm{w}^{2}}}$ & $\begin{array}{c}T_{\text {hot }} \\
(\mathrm{keV})\end{array}$ & $\begin{array}{l}f_{\text {hot }} \\
(\%)\end{array}$ & $\begin{array}{c}\text { Shock velocity } \\
u_{\mathrm{s}} \\
(\mathrm{cm} / \mathrm{s})\end{array}$ & $\begin{array}{c}p_{\text {abl }} \\
\text { (Mbar) }\end{array}$ & $\begin{array}{c}T_{\mathrm{R}} \\
(\mathrm{eV})\end{array}$ & $\begin{array}{c}\text { Dante } \\
T_{\mathrm{R}} \\
(\mathrm{eV})\end{array}$ \\
\hline 94032704 & 0.64 & 1.52 & 0.295 & 3.517 & 30 & 0.2 & $4.16 \times 10^{6}$ & 36 & 176 & 146 \\
\hline $94032707^{a}$ & 0.50 & 1.62 & 0.344 & 2.048 & - & 0.06 & $5.15 \times 10^{6}$ & 53 & 198 & 160 \\
\hline $94032807^{a}$ & 0.64 & 1.52 & 0.314 & 3.460 & 31 & 0.2 & $4.38 \times 10^{6}$ & 38 & 181 & 149 \\
\hline 94032903 & 0.50 & .1 .38 & 0.348 & 2.016 & 30 & 0.2 & $5.15 \times 10^{6}$ & 53 & 198 & 169 \\
\hline $94032907^{b}$ & 0.33 & 1.51 & 0.168 & 1.030 & 27 & 0.15 & $5.07 \times 10^{6}$ & 52 & 197 & 156 \\
\hline $94033011^{c}$ & 0.50 & 1.50 & 0.334 & 1.946 & 26 & 0.31 & $3.69 \times 10^{6}$ & 27 & 164 & 155 \\
\hline 94041304 & 0.80 & 1.1 & 0.548 & 5.257 & 27 & 0.03 & $2.99 \times 10^{6}$ & 18 & 145 & 121 \\
\hline $94041307^{a}$ & 0.33 & -1.35 & 0.178 & 0.992 & 45 & 0.044 & - & - & - & 178 \\
\hline 94042604 & 0.50 & 1.63 & 0.395 & 1.905 & 29 & 0.08 & $5.75 \times 10^{6}$ & 66 & 210 & 175 \\
\hline
\end{tabular}

a Partial miss, but most of the energy went inside.

bBad miss.

Maybe a bad miss; low drive, but no $8 \times$ microscope picture. 
Radiation-Drive Scaling at $2 \omega$ and $4 \omega$

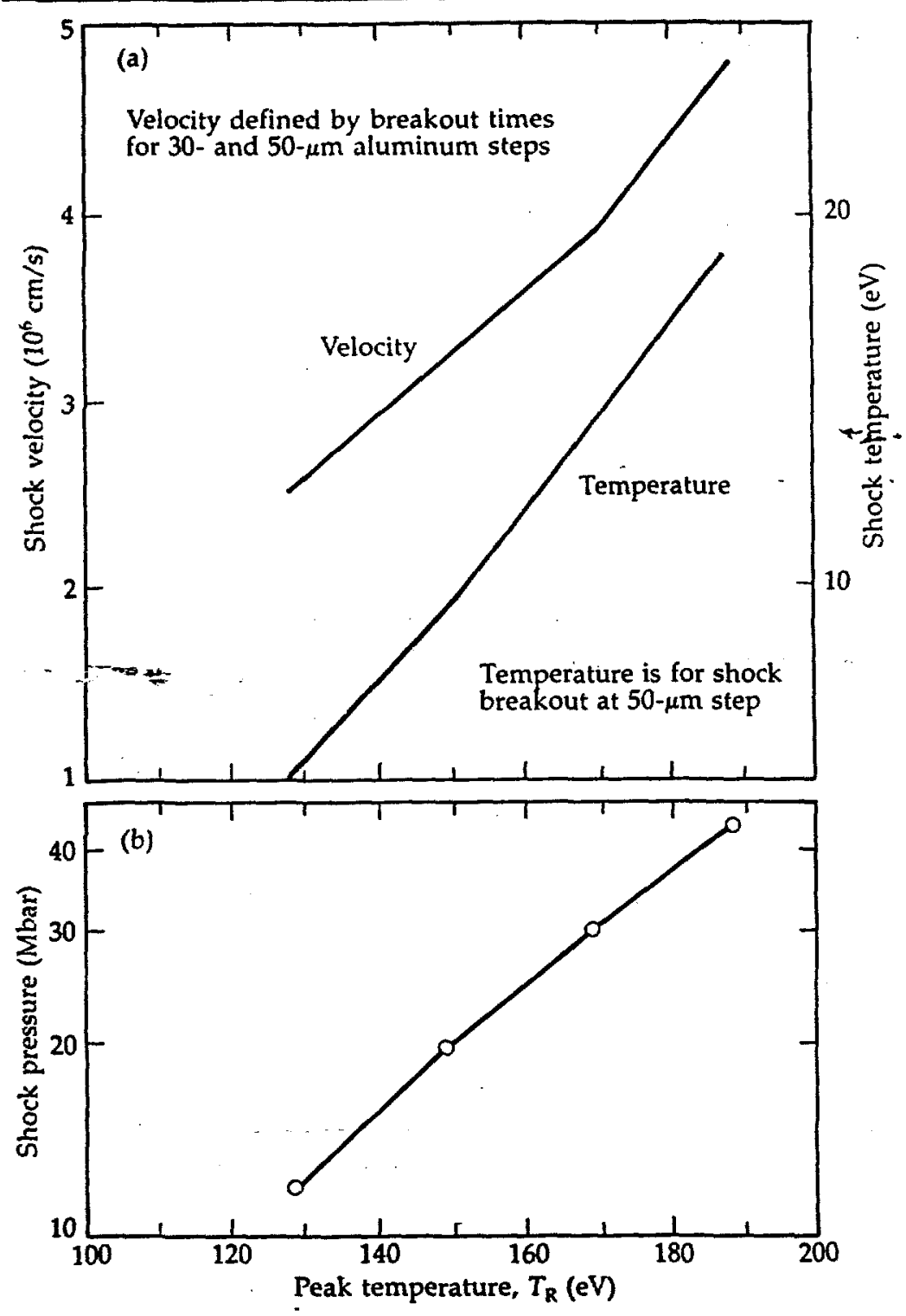

LASNEX includes some time-averaging of the radiation drive.

The witness-plate measurements for both 5270 - and $2630-\AA$ irradiation agree quite well with the corresponding radiation temperatures calculated from the Marshak scaling law given in Eq. (3) with $\eta=0.8$

(Fig. 4-10). Not plotted are those measurements for which the streak camera was badly out of focus (200-ps and 200- $\mu \mathrm{m}$ resolutions) or for which the preheat was high. Also not plotted are the measurements from shot Nos. 94032907 and 94033011 because of probable severe clipping of the laser beam at the laser entrance hole.

According to the shock-velocity measurements, the radiation drive appears to be insensitive to whether the irradiation wavelength is 5270 or $2630 \AA$, whereas the Dante measurements (Fig. 4-11) show that $5270-\AA$ irradiation gives a slightly higher drive; however, the scatter in these data makes this conclusion highly uncertain. Note that the calculated Marshak temperature in Fig. 4-11 uses $\eta=0.5$, rather than $\eta=0.8$ that was used in Fig. 4-10, since the value of 0.5 is more consistent with the Dante measurements. The value of 0.5 is also more consistent with the values for $E_{\mathrm{rad}} / E_{\mathrm{abs}}$ that were measured for gold-disk targets irradiated with 5320 - and $3547-\AA$ light at Argus. (Although those Argus experiments were at $600 \mathrm{ps}$, the pulse-length dependence of $E_{\mathrm{rad}} / E_{\mathrm{abs}}$ is believed to be weak, e.g., $\approx \tau^{0.07}$.)

The shock breakout usually, though not always, showed a bright initial flash that quickly dimmed. Figure 4-12(a) summarizes an experiment in which a 1.0 -scale halfCairn was irradiated with $5270-\AA$ light. Figure 4-12(b) shows a color-enhanced photo of a streak record for a witness plate in this experiment, and Fig. 4-12(c) contains lineouts showing the shock breakouts. The flash was most pronounced for this experiment and for a 0.8-scale half-Cairn irradiated with $2630-\AA$ light. These were also the two lowest drive experiments, which generated the weakest shocks. The shock rise times were also fairly fast, but the fastest shock breakout, 23 ps, was observed for a 0.50 -scale half-Cairn irradiated with $2630-\AA$ light.

If there is no preheat, the blackbody temperature at the peak of the flash is interpreted as being the temperature behind the shock front in the dense material. The 
flash duration is determined both by the Riemann invariant $u+\int\left(d p / \rho c_{s}\right)$ of the heated aluminum and by its opacity at the wavelength of the observed light. The distance we can see into the blowoff plasma is determined by the equation

$$
\int_{x}^{\infty} k d x=1 .
$$

If the opacity is high, then, after only a short period of unloading, we can see only into regions of very low density. These regions have been adiabatically cooled, explaining the flash.

If there is preheat, the situation is more complicated. A low-density blowoff plasma forms and may prevent us from seeing the dense inaterial. The shock breakout time is modified not only because its Hugoniot is

(a) Experiment summary

Target:

1.0-scale half-Cairn No. 177

$1314 \mu \mathrm{m} \mathrm{L}, 1552 \mu \mathrm{m}$ D, $755 \mu \mathrm{m}$ LEH

Laser beam:

$1.95 \mathrm{~kJ}, 5270 \AA$, $1 \mathrm{~ns}, \mathrm{f} / 4.3$ diverging beam

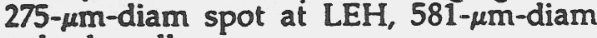
at back wall

Aluminum witness plate:

30- $\mu \mathrm{m}$ step, 590 ps; $48-\mu \mathrm{m}$ step, 1120 ps

$u_{\mathrm{s}}=3.33 \times 10^{6} \mathrm{~cm} / \mathrm{s}$

$\Rightarrow p=21 \mathrm{Mbar}, T_{R}=152 \mathrm{eV}$

FFLEX: $E_{\text {hot }}=12.5 \mathrm{~J}, T_{\text {hot }}=22.4 \mathrm{keV}$

Dante: $T_{R}=135 \mathrm{eV}$ changed but also, and more importantly, because the shock may have to travel far down the density gradient before it is observable. The optical emission from the shock breakout is not just delayed,

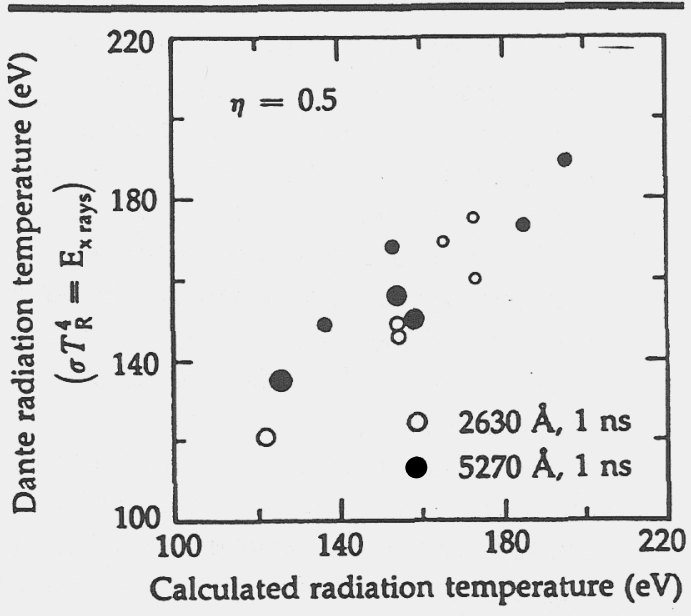

(b) Streak record

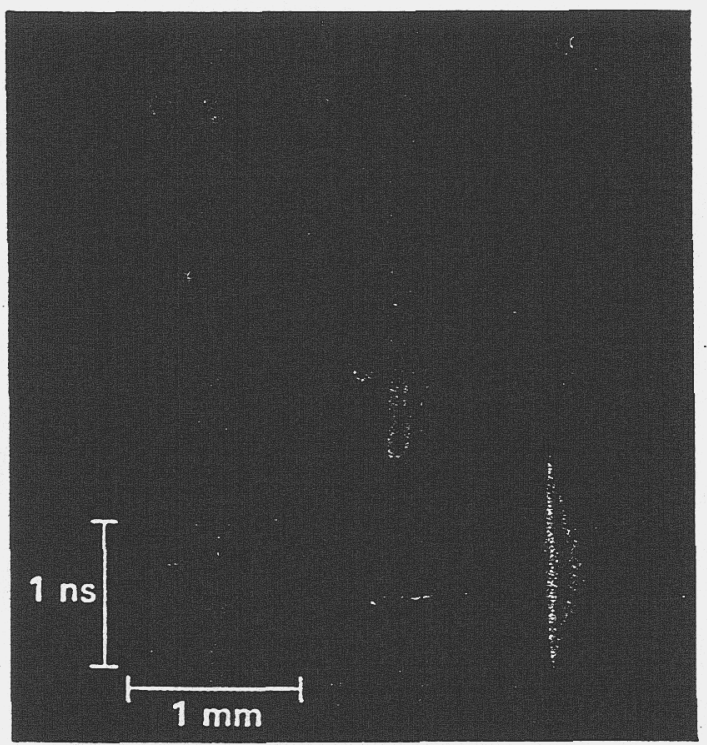

Fig. 4-11. Dante measurements of the radiation temperature for empty gold halfCairns. Circle sizes are proportional to hohlraum sizes, which were 0.50 -, 0.64-, 0.80-, and 1.0-scale.
Fig. 4-12. Shockvelocity measurement of the radiation drive. (a) Experiment summary. (b) Colorenhanced streak record. (c) Lineouts showing the shock breakouts. (c) Lineouts

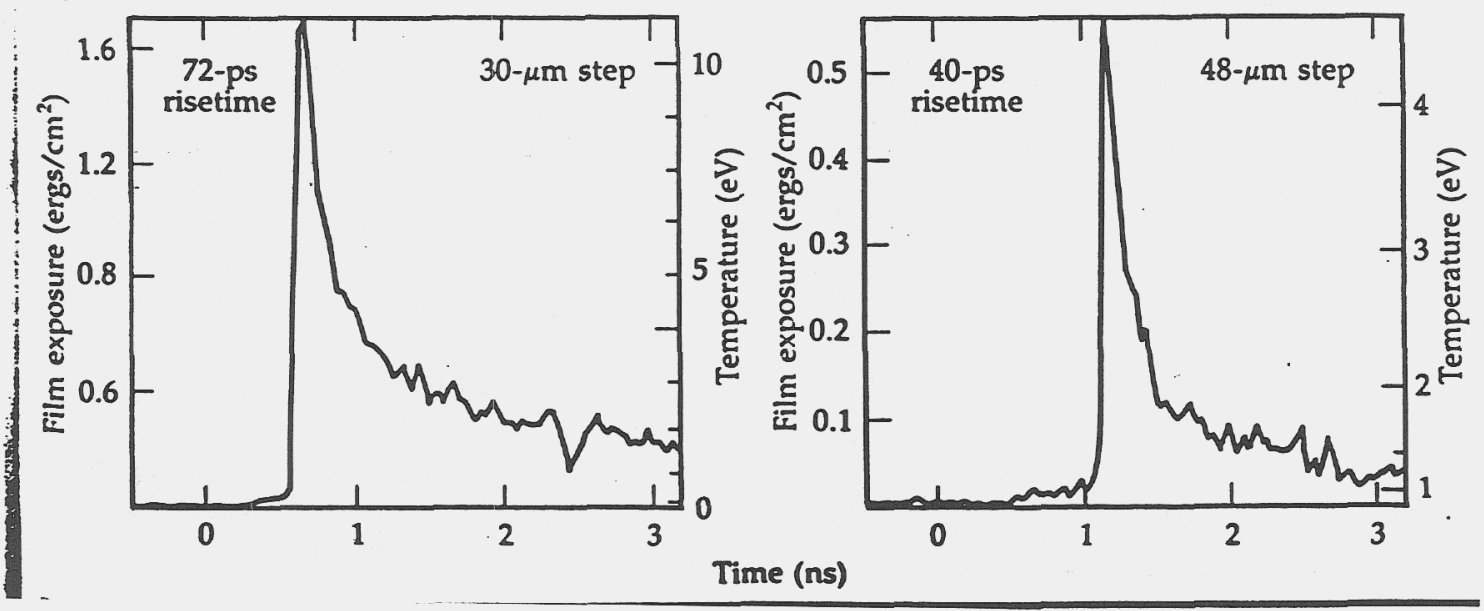


Radiation-Drive Scaling at $2 \omega$ and $4 \omega$

however. The temperature behind the shock is increased because it travels down a density gradient, and both the rise and fall times of the flash are also increased.

Some shocks did not show a flash, contrary to our expectations. Although our understanding is evidently incomplete, the point remains that the observed temperature is not necessarily the temperature behind the shock in the dense material.

One point that has been glossed over so far is that the shock wave will decay as the radiation drive diminishes. For instance, at the time of the second shock breakout for shot No. 94042604, it is estimated that the $\sigma T_{\mathrm{R}}^{4}$ drive is only $70 \%$ of its maximum value, which occurs 400 ps after the peak of the laser pulse (see Table 4-5, which is discussed later in this article under the heading "LASNEX Modeling"). However, the head of the rarefaction wave requires time to reach the back surface, and we will show this time to be substantial.

The head of the rarefaction wave travels at sound speed in the compressed and heated aluminum behind the shock. Along the principal Hugoniot, the sound speed, $C_{s}$, is given by the fit

$C_{s}=0.74 \times 10^{6}+0.325 \times 10^{6} \sqrt{P(M b)} \mathrm{cm} / \mathrm{s}$

which is $1 \%$ accurate for $1 \mathrm{Mbar}<P<100$ Mbar. The density, $\rho$, is given by the fit

$\rho=\frac{\rho_{0}+14 X}{1+X}, X=A \cdot P+\frac{1}{B}-\frac{1}{B+P}$

where $P$ is in Mbar and the constants of the fit are $\rho_{0}=2.786 \mathrm{~g} / \mathrm{cm}^{3}, A=0.036$, and $B=2.5$. This latter equation is $2 \%$ accurate for $0.1 \mathrm{Mbar}<P<500 \mathrm{Mbar}$. For $P=66 \mathrm{Mbar}, C_{\mathrm{s}}=3.38 \times 10^{6} \mathrm{~cm} / \mathrm{s}$, and the

Table 4-5. LASNEX calculations for radiation temperature near the witness plate for a 0.50 -scale gold halfCairn irradiated with $1.5 \mathrm{~kJ}$ of $2630 \mathrm{~A}$ light in 1 ns.

\begin{tabular}{cc}
\hline $\begin{array}{c}\text { Time relative to } \\
\text { peak of laser pulse } \\
\text { (ns) }\end{array}$ & $\begin{array}{c}\text { Peak radiation temperature } \\
\text { along } K=10 \text { mesh line } \\
(\mathrm{eV})\end{array}$ \\
\hline 0 & 204 \\
0.1 & 208 \\
0.2 & 214 \\
0.3 & 217 \\
0.4 & 220 \\
0.5 & 219 \\
0.6 & 215 \\
0.7 & 211 \\
0.8 & 202 \\
\hline
\end{tabular}

$68.8-\mu \mathrm{m}$ step is compressed to $17.5 \mu \mathrm{m}$, giving a transit time of 520 ps. Even though the shock breakouts occur long after the peak of the laser pulse, the shocks are at their strongest at the time of breakout.

\section{Error Analysis}

The shock-velocity measurements are almost certainly accurate to $\pm 15 \%$. The sources of error under our control include the measurements of the thickness and density of the aluminum steps and the calibration of the streak-camera sweep speed. Other error sources include the aluminum equation of state, the modeling of the aluminum ablation, and the preheat. Only those shock-velocity-medsurements with little observable preheat have been used to infer the drive; the limit of preheat observability is in the range of 1 to $1.5 \mathrm{eV}$. Our FFLEX measurements indicate that, for all the $4 \omega$ experiments and for most of the $2 \omega$ experiments, the preheat due to suprathermal electrons diffusing through the aluminum should have been much less than $1 \mathrm{eV}$.

The first source of error under our control is measurement of the thickness and density of the steps on the aluminum witness plates. A scanning-electron-microscope picture of a bent witness plate is shown in Figure 4-13. The witness plate was made by masking and etching evaporated aluminum, and the step heights were measured to an accuracy of $\pm 1 \mu \mathrm{m}$ using filtered white light with a short coherence length (10 to 15 fringes). During measurement, light reflected from the witness plate interfered with a reference; then, when the witness plate was moved up and down, fringes could be seen at one position for one step and at another position for the other step. The step height was then determined by a vernier on the microscope, which had been accurately calibrated by fringe counting. For most of the $2 \omega$ experiments; the step height was $24 \mu \mathrm{m} \pm 4 \%$; for the $4 \omega$ experiments, step heights ranged from $20 \pm 5 \%$ to $36 \pm 3 \% \mu \mathrm{m}$.

Hsieh measured a density of $2.72 \pm 0.11$ $\mathrm{g} / \mathrm{cm}^{3}(1 \sigma)$ for the evaporated aluminum he used to make the witness plates. This standard deviation is the result of measurements on a number of samples. Most likely. 
Fig. 4-19. "Megaphone" 0.8-scale Cairn used to make the shock-velocity measurement of the drive at the center of the secondary.
Fig. 4-20. Shockvelocity measurement of the radiation drive in the secondary. (a) Experiment summary. (b) Color-enhanced streak record. (c) Lineouts showing the shock breakouts.

Radiation-Drive Scaling at $2 \omega$ and $4 \omega$

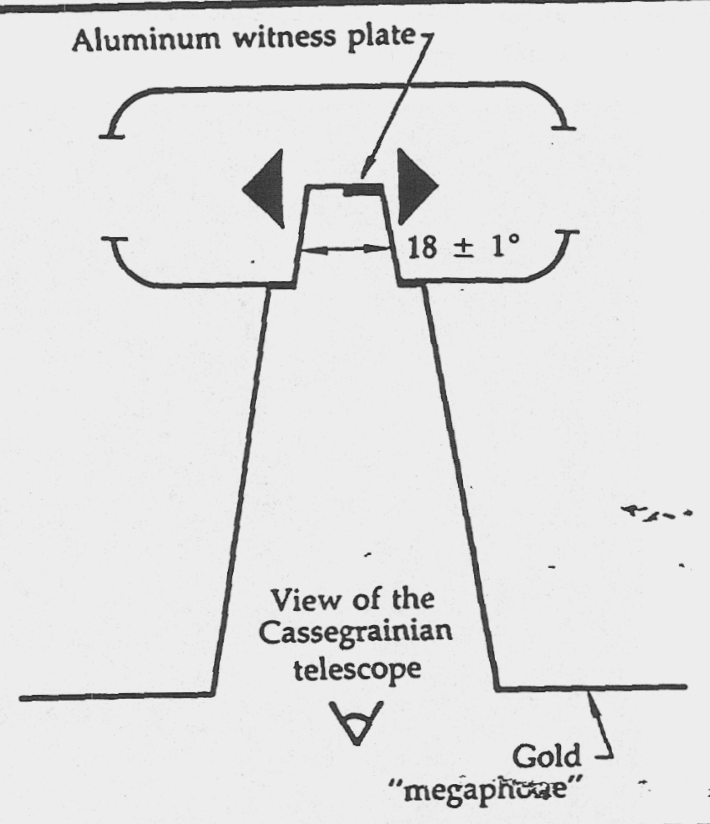

(a) Experiment summary

Target:

0.8-scale "megaphone" Cairn No. 402

$2487 \mu \mathrm{m}$ L, $1198 \mu \mathrm{m} \mathrm{D}, 696 \mu \mathrm{m}$ LEH

Laser beam:

$7.2 \mathrm{~kJ}, 5270 \AA \AA, 1 \mathrm{~ns}, f / 4.3$ diverging beam $278-\mu \mathrm{m}$-diam spot at LEH, 600- $\mu \mathrm{m}$-diam at cone

Aluminum witness plate:

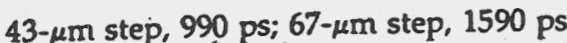

$u_{\mathrm{s}}=4.0 \times 10^{6} \mathrm{~cm} / \mathrm{s}$

${ }_{s} p=32 \mathrm{Mbar}, T_{\mathrm{R}}=172 \mathrm{eV}$

FFLEX: $200 \mathrm{~J}$ of $34-\mathrm{keV}$ electrons $\Rightarrow$

$T_{\text {preheat }}=1.3 \mathrm{eV}$ for $43-\mu \mathrm{m}$-step,

$T_{\text {preheat }}=0.3 \mathrm{eV}$ for $67-\mu \mathrm{m}$ step

Dante: $T_{R}=155 \mathrm{eV}$

(c) Lineouts

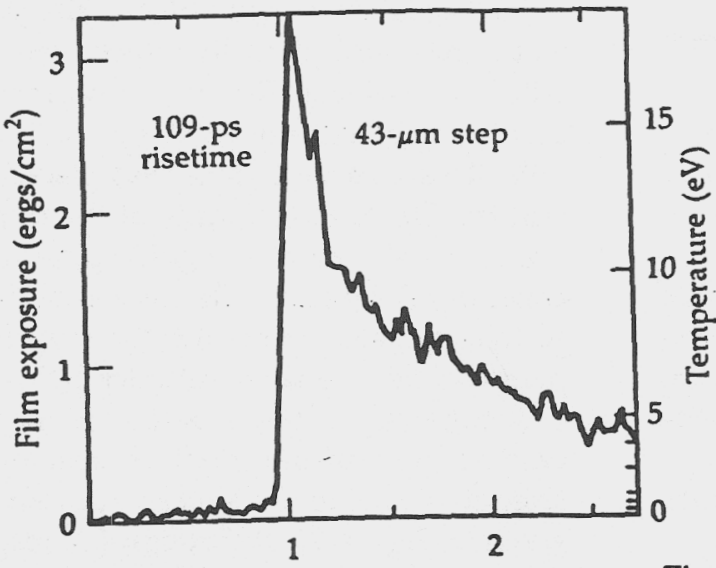

gave a radiation temperature of $172 \mathrm{eV}$ for the secondary, whereas the Dante measured a temperature of $155 \mathrm{eV}$ for the primary. As shown in Table 4-3, the radiation temperatures inferred from the Dante measurements are typically about $17 \mathrm{eV}$ lower than those inferred from the shock-velocity measurements (ignoring the high-preheat shots). Thus, the primary and secondary in this experiment were probably about the same temperature. This conclusion is in agreement with the WALLE calculations of Suter, ${ }^{15}$ which indicate that the radiation temperature near the witness-plate position at the center of the secondary should be 1 to $3 \mathrm{eV}$ hotter than that area of the primary wall seen by Dante $H$.

Comparison of these results with other experimental data is difficult, however.

(b) Streak record
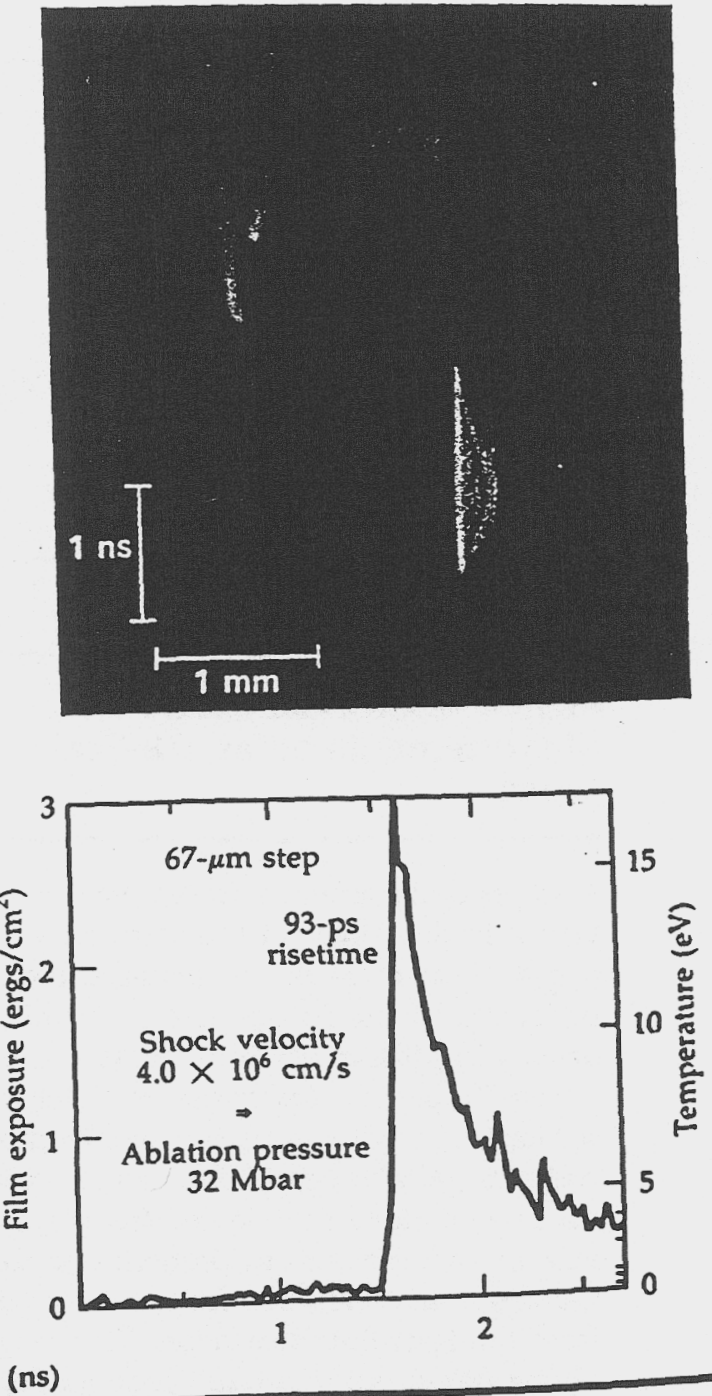
Radiation-Drive Scaling at $2 \omega$ and $4 \omega$

(a) Experiment summary

Target:

0.64 -scale half-Cairn No. 180

$838 \mu \mathrm{m}$ L, $996 \mu \mathrm{m} \mathrm{D}, 548 \mu \mathrm{m}$ LEH

Laser beam:

$3.4 \mathrm{~kJ}, 5270 \AA, 1 \mathrm{~ns}, \mathrm{f} / 4.3$ diverging beam

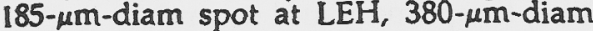
at back wall

Aluminum witness plate:

43- $\mu \mathrm{m}$ step, 570 ps; $67-\mu \mathrm{m}$ step, 1155 ps Shock-velocity measurement invalid because of high preheat: $10.2 \mathrm{eV}$ for 43- $\mu \mathrm{m}$ step, $3.8 \mathrm{eV}$ for $67-\mu \mathrm{m}$ step

FFLEX: $625 \mathrm{~J}$ in $31 \mathrm{keV}$ suprathermal electrons $\approx$ preheat temperatures close to those measured optically

Dante: $T_{R}=173 \mathrm{eV}$

(c) Lineouts

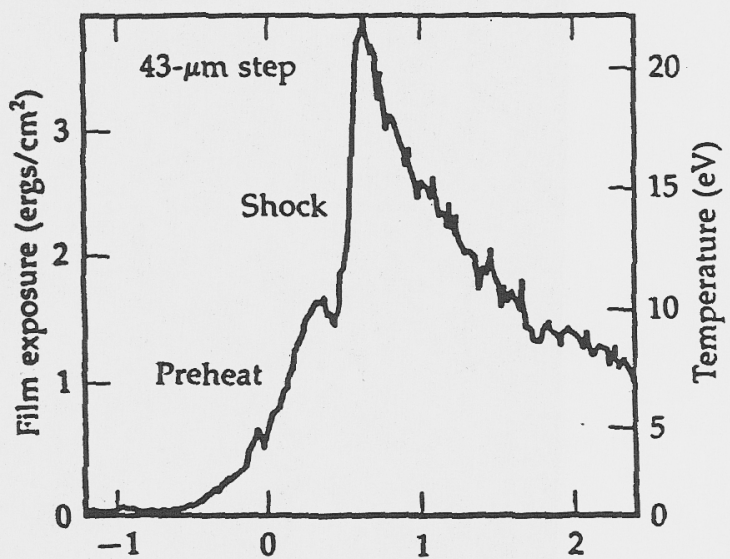

Time (ns)

(b) Streak record

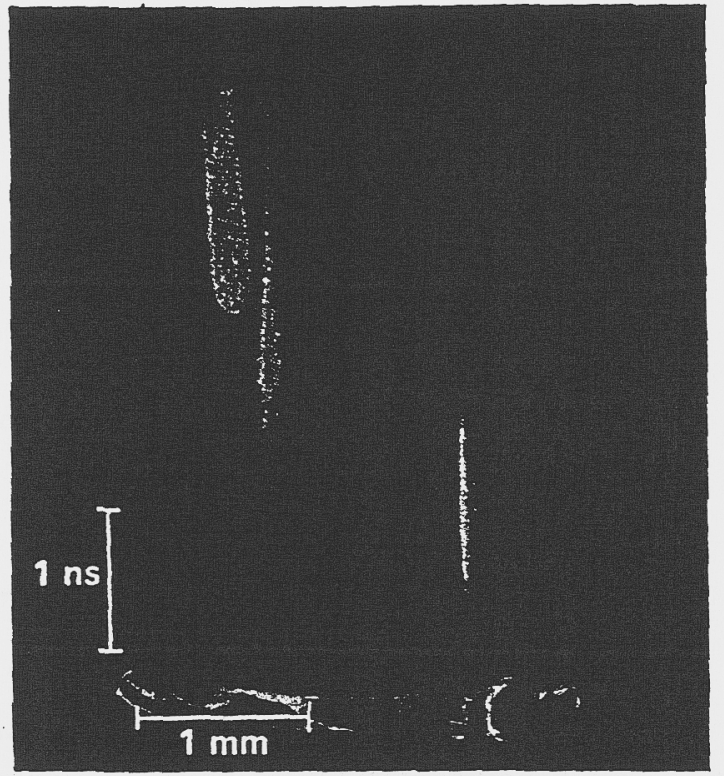

Fig. 4-18. Witnessplate experiment with high preheat. (a) Experiment summary. (b) Color-enhanced streak record. (c) Lineouts showing the shock breakouts.

\section{Primary/Secondary Drive Measurements on an 0.8-Scale Cairn}

A stepped aluminum witness plate was placed in the center of the secondary (Fig. 4-19) to measure the actual drive experienced by the fuel pellets in the highdensity implosion experiments (see the article "Implosion Experiments" later in this section). The witness plate was glued to the end of a gold "megaphone," which simultaneously shielded the viewed (rear) steppedaluminum surface from preheat and the Cassegrainian telescope from seeing plumes from the laser entrance holes. The megaphone flared out in an $18^{\circ}$ cone angle, since

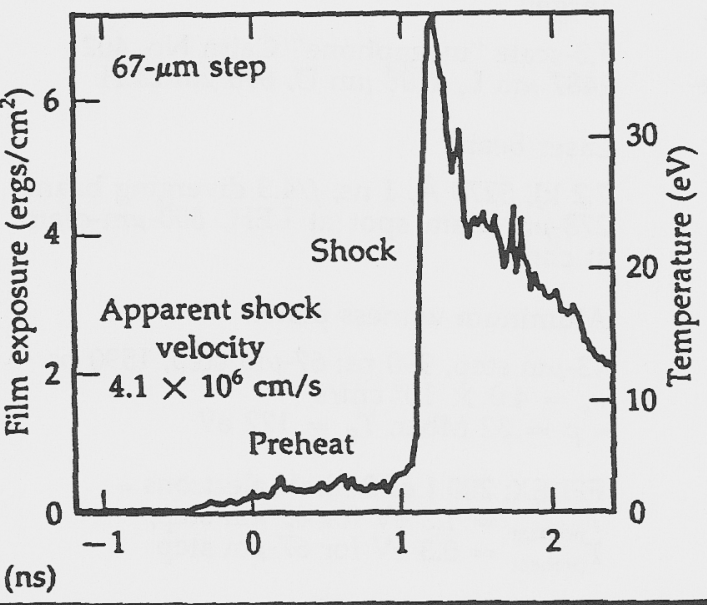

the $f / 4.7$ Cassegrainian telescope collected light over a $12^{\circ}$-diam cone. There was allowance for a $3^{\circ}$ misalignment.

The radiation temperature in the primary was measured by Dante, which was placed with a line of sight $20^{\circ}$ to the normal so that it could see only its $400-\mu \mathrm{m}$ diagnostic hole. This diagnostic hole was placed so that the Dante looked only at the opposite primary wall, not at the gold scattering cone. In addition, the Dante was shielded so that it could see neither the strip nor the plumes from the laser entrance holes.

A summary of the experiment, a color enhancement of the streak record, and lineouts showing the shock breakouts are given in Figs. 4-20(a) through (c), respectively. The shock-velocity measurement 
Fig. 4-17. TDG picture looking across the laser entrance hole. A $200-\mathrm{V}$ backlighter replaced the back of the hohlraum at $z=300 \mu \mathrm{m}$
Radiation-Drive Scaling at $2 \omega$ and $4 \omega$

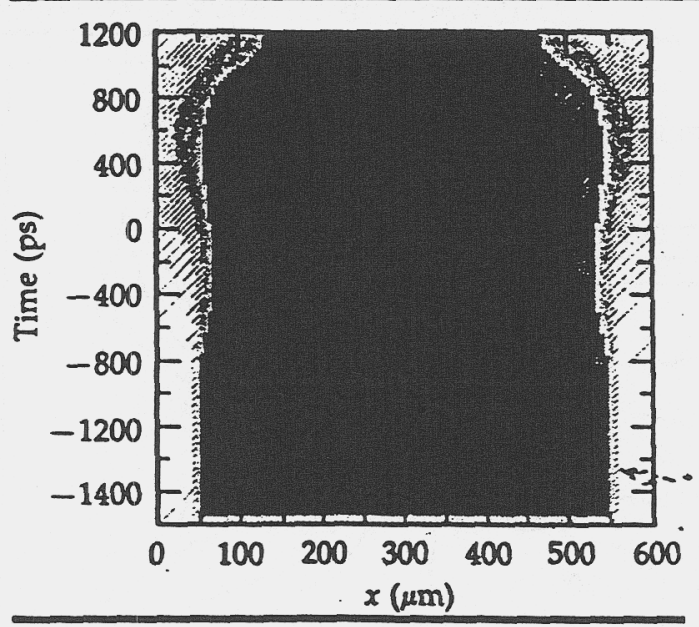

LASNEX TDG postprocessor was run with a line of sight along the $z$ axis looking at the laser entrance hole. A 200-eV blackbody backlighter was placed at $z=300 \mu \mathrm{m}$, replacing the rear of the Caim. The TDG picture (Fig. 4-17) has one edge of the laser entrance hole at $x=0.005 \mathrm{~cm}$, has the other edge at $x=0.055 \mathrm{~cm}$, and has the peak of the laser pulse at $t=0$. The edges of the four shaded regions are at $72,36,18$, and $9 \%$, going from darkest to lightest. The effective hole closure is about $25 \mu \mathrm{m}$ in the radius. Weber stresses that what we have is a pressure balance between the relatively dense cold material moving inward from the edge of the laser entrance hole and the hot rarefied plasma that flows out of the laser entrance hole at high velocity $\left(10^{8} \mathrm{~cm} / \mathrm{s}\right)$. Effective closure of the Dante hole by $50 \mu \mathrm{m}$ in the diameter would cause Dante to underestimate the temperature of a $200-\mathrm{eV}$ hohlraum by $13 \mathrm{eV}$ for a $400-\mu \mathrm{m}$ diam diagnostic hole and by $17 \mathrm{eV}$ for a 300- $\mu$ m-diam diagnostic hole.

\section{Preheat}

Optical emission before the shock breakout is a signature of preheat. For one experiment (shot No. 94020909), such emission was clearly visible for both steps on the witness plate. The suprathermal electron temperature can be obtained from the ratio of the preheat in the two steps. Obtaining the total $f_{\text {hot }}$ requires that some assumptions be made. We assume that the suprathermal electron flux on the hohlraum wall is both isotropic and Maxwellian in energy. We also assume that the suprathermal electron energy deposition is uniform over the entire inside surface of the Caim and that any electrons that diffuse through to the outside boundary must return due to the target potential. This last assumption means that the preheat at the back of the aluminum witness plate is double what it would be if the target potential were zero.

We use the approximate analytical formulas given by Harrach and Kidder ${ }^{13}$ for electron-energy deposition. These formulas are based on Monte Carlo calculations and use the formalism developed by Spencer.14 The experimental observable is the timeresolved optical brightness of the back surfaces of the two aluminum steps. Since the brightness is measured absolutely, we can calculate the blackbody temperatures. Using the computer code XES and the equationof-state tables EOP811, we first calculate the specific energy deposition and then obtain $W_{\text {abs }}$ by using the formula

$E_{\text {dep }}(l)=\frac{W_{\text {abs }}}{r_{0}\left(k T_{\mathrm{h}}\right)} \frac{\beta^{2}}{2} \exp \left(-\beta \sqrt{\frac{\rho_{0} l}{r_{0}\left(k T_{h}\right)}}\right)$.

The electron range is

$r_{0}(E)=b E^{1+\mu}$.

The parameter values for aluminum are $\beta=2.17, \mu=0.72$, and $b=6.77 \times 10^{-6}$ $\left(\mathrm{g} / \mathrm{cm}^{2}\right) /(\mathrm{keV})^{1+\mu}$. Here, $W_{\text {abs }}$ is the suprathermal electron energy deposition per unit area and $l$ is the depth in the material. We will halve the value for $W_{\text {abs }}$ that we get from these formulas to account for electron trapping by the target potential.

For shot No. 94020909, we measured a temperature of $10.2 \mathrm{eV}$ for the $43-\mu \mathrm{m}$ step and $3.8 \mathrm{eV}$ for the $67-\mu \mathrm{m}$ step. These results imply specific energy depositions of $2.7 \times 10^{12}$ and $0.84 \times 10^{12} \mathrm{ergs} / \mathrm{g}$, respectively. A density of $\rho=0.1$ was used. From the foregoing information, we calculate $T_{\text {hot }}=31 \mathrm{keV}$ and $0.5 W_{\text {abs }}=165 \mathrm{~J} / \mathrm{mm}^{2}$. Since $A=3.8 \mathrm{~mm}^{2}$, the total energy in suprathermal electrons should be about 610 J. Our FFLEX measurements imply 625] in a $31-\mathrm{keV}$ electron distribution. The shot description is given in Fig. 4-18(a), the streak record is shown in Fig. 4-18(b), and the lineouts showing shock breakouts are given in Fig. 4-18(c). 
Radiation-Drive Scaling at $2 \omega$ and $4 \omega$

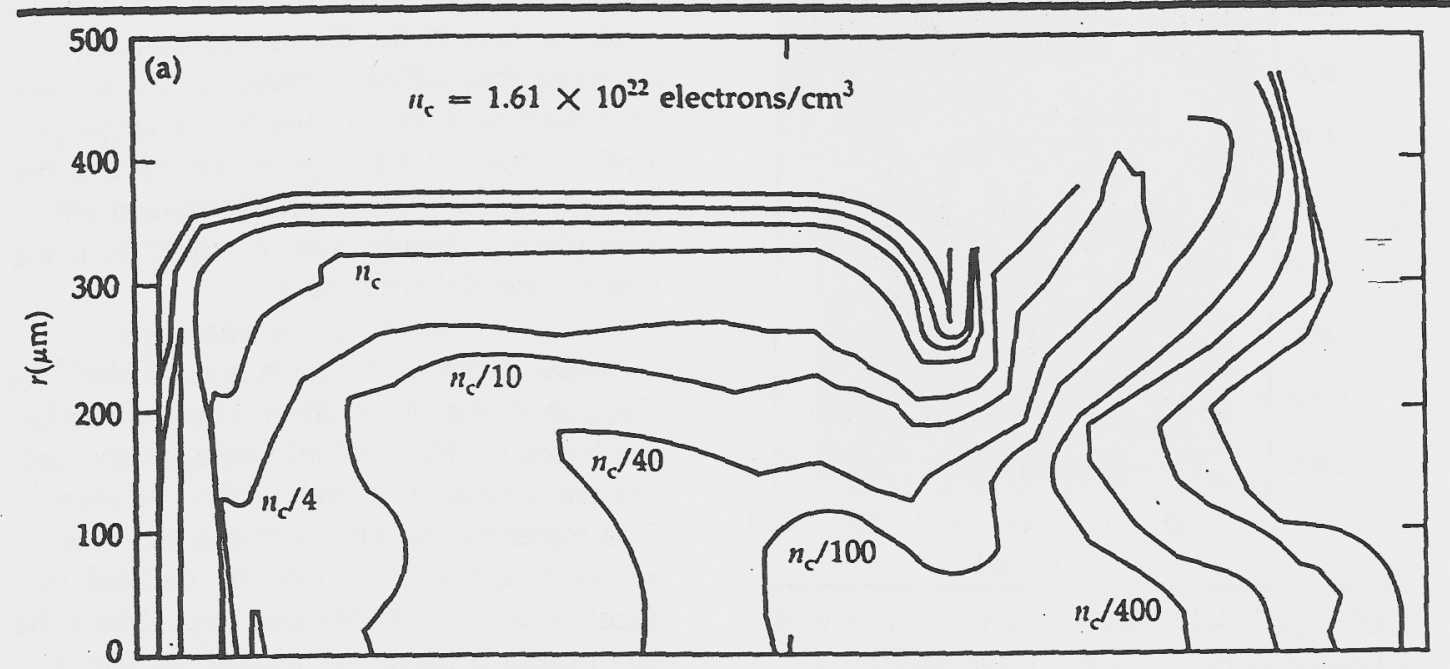

Fig. 4-15. (a) Density and (b) radiation-

temperature contours at the peak of the laser pulse for a

LASNEX simulation of a 0.5 -scale half-Cairn irradiated with $1.5 \mathrm{~kJ}$ of 2630-A light in 1 ns.

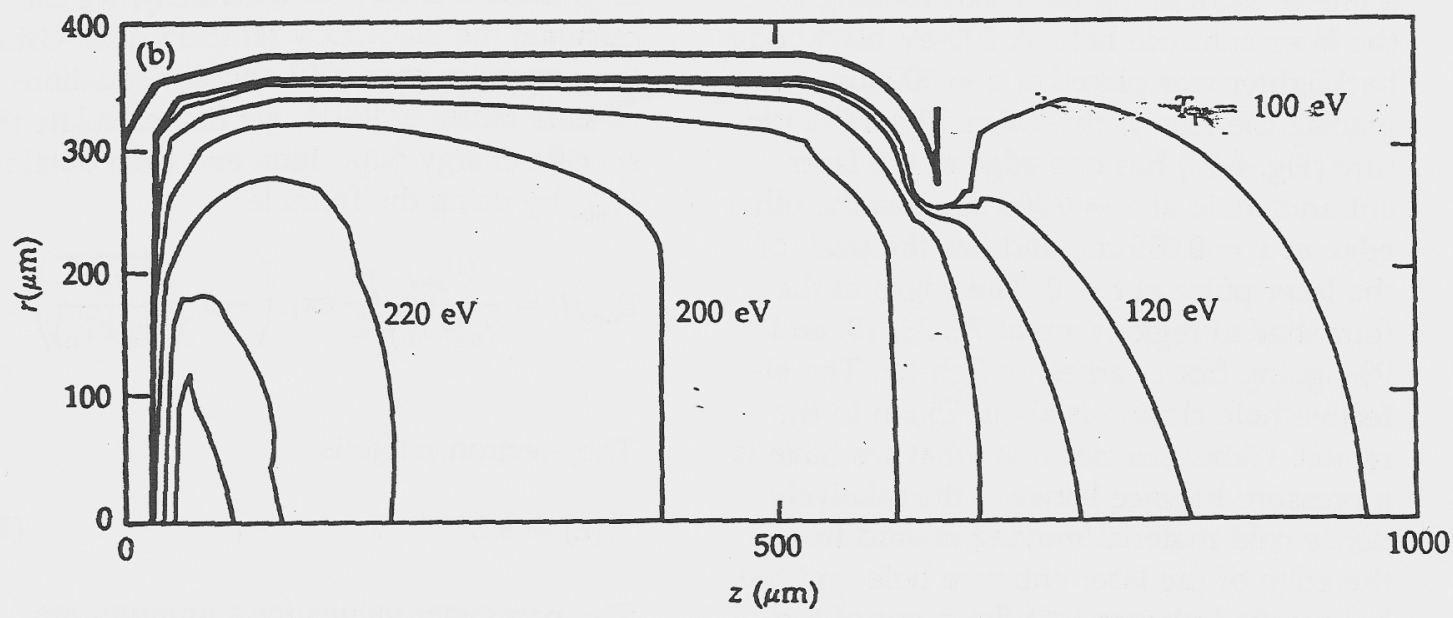

Fig. 4-16. Conditions along the $K=10$ mesh line at 400 ps after the peak of the laser pulse for a LASNEX simulation of a 0.5 -scale half-Cairn irradiated with $1.5 \mathrm{~kJ}$ of 2630-A light in $1 \mathrm{~ns}$.

near the wall at $z=300 \mu \mathrm{m}$. These values are averages of the incident and reradiated $x$-ray flux. If we define $T_{R}$ on the basis of the $\sigma T_{R}^{4}$ flux passing through an area parallel to the wall, then, at this time, the $T_{R}$ using only the reradiated flux is 5 to $10 \mathrm{eV}$ lower than the $T_{R}$ based on the incident flux. At times much after the peak of the laser pulse, this difference goes to zero.

The drive peaks about 400 ps after the peak of the laser pulse (when it peaks depends upon where it is measured). Figure 416 shows how $T_{R}, p$, and $T_{c}$ vary along the $K=10$ mesh line at this time. The Dante diagnostic hole and witness plate were at about $z=300 \mu \mathrm{m}$ for the experiments. The $K=10$ mesh line starts at $z=300 \mu \mathrm{m}$ on the side wall and intercepts the $z$ axis at $z=446 \mu \mathrm{m}$ (see Fig. 4-14). The peak $T_{R}$ of $220 \mathrm{eV}$ can be compared to Rosen's scaling i relation, Eq. (3), which predicts $T_{\mathrm{R}}=182 \mathrm{eV}$ for $\eta=0.5$ and $T_{R}=210 \mathrm{eV}$ for $\eta=0.8$.

To get a crude idea of the effect of hole closure on the Dante measurement, the

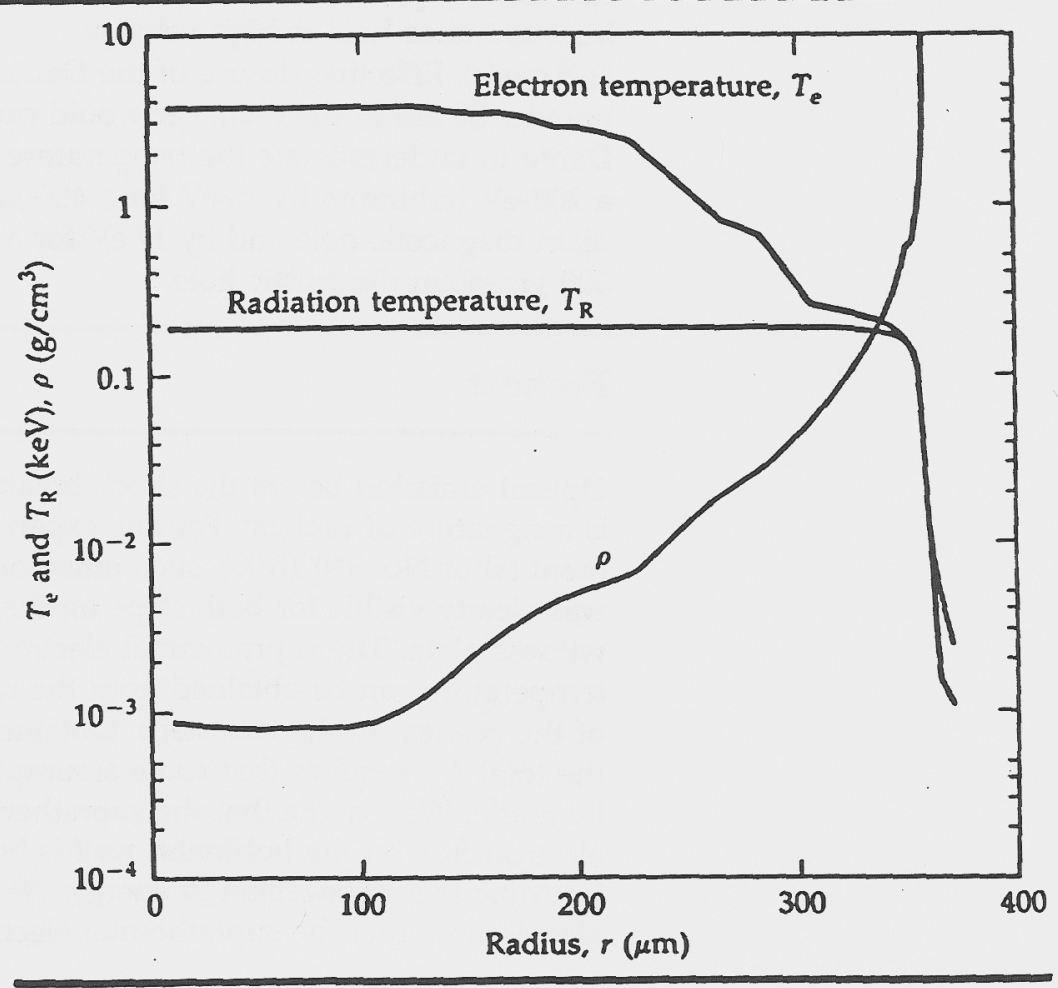


Radiation-Drive Scaling at $2 \omega$ and $4 \omega$

Fig. 4-14. Eulerian mesh for the LASNEX simulation of a 0.5 scale half-Cairn irradiated with $1.5 \mathrm{~kJ}$ of 2630-Â light in $1 \mathrm{~ns}$.

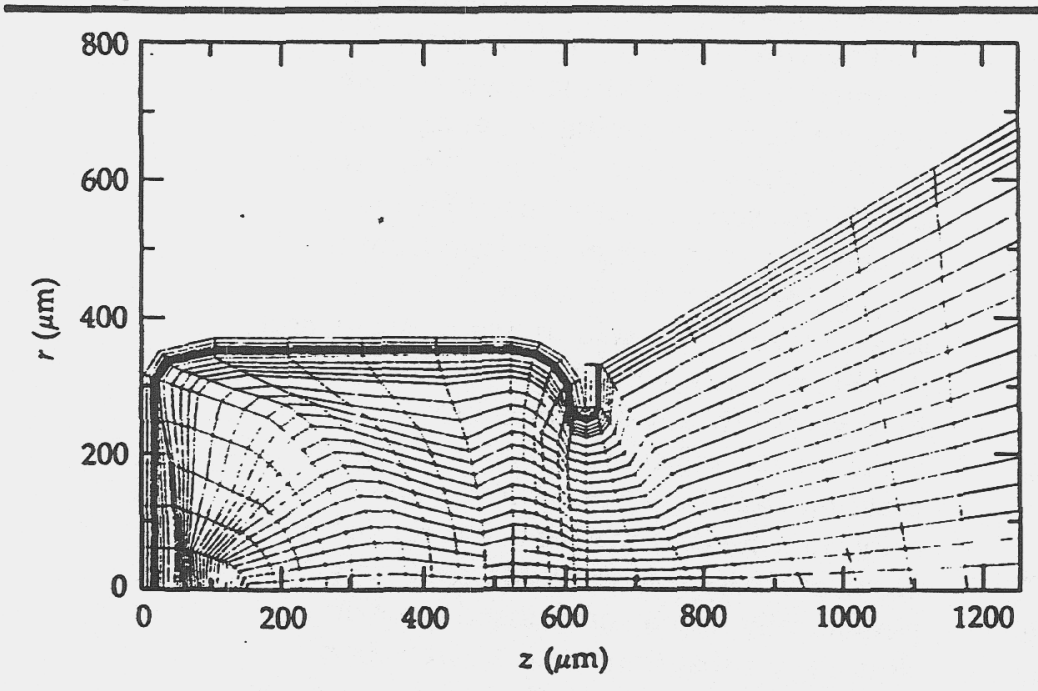

For all our experiments, the radiation temperature was much below the critical temperature for aluminum $(425 \mathrm{eV})$.

Hatchett states that his analysis is valid only for materials with atomic number $Z<7$. However, for $T_{R}<200 \mathrm{eV}$, aluminum should be much like a hydrocarbon material $(\mathrm{CH})$. The $1 \mathrm{~s}$ electrons in aluminum will not be stripped, whereas the $n=2$ electrons have comparable binding energies to the 1s electrons in carbon. Most of the energy in the radiation field is at energies below the aluminum $\mathrm{K}$-edger-so that the bound-electron contribution to. the opacity of helium-like aluminum will be small.

Hatchett's analysis does not account for the ionization energy, which is large for aluminum. However, it is simple to generalize his analysis for the expansion heat front. When the radiation temperature is much less than the critical temperature, we find

$$
P_{\mathrm{abl}} \approx \frac{\sigma T_{\mathrm{R}}^{4}}{\sqrt[2]{\frac{N_{\mathrm{A}} k T_{\mathrm{R}}}{\mu}}} \cdot \frac{1}{1+\frac{\sum_{\alpha} f_{\alpha} E_{\alpha}}{2 k T_{\mathrm{R}}}}
$$

the summed binding energy of all the removed electrons.

For helium-like aluminum, $\mu=27 / 12$ and $f\left(\mathrm{Al}^{+11}\right)=1 / 12$. We find $E\left(\mathrm{Al}^{+11}\right)=2214 \mathrm{eV}$ from the table of ionization energies. ${ }^{12}$ For $T_{R}=200 \mathrm{eV}$, we find $P_{\text {abl }} \approx 61 \mathrm{Mbar}$, which is about $11 \%$ higher than the LASNEX result of $55 \mathrm{Mbar}$.

\section{LASNEX Modeling}

Design calculations for the witness plates are discussed earlier in this report (see the article "Burn-Through Foil and WitnessPlate Experiments for Novette" in Sec. 2). Modeling of the hohlraum environment is in progress and will be reported in more detail in the future. One preliminary result obtained Weber is a two-dimensional model of a 0.5-scale half-Cairn irradiated with $1.5 \mathrm{~kJ}$ of $2630-\AA$ light in $1 \mathrm{~ns}$. Inside, the Caim is $590 \mu \mathrm{m}$ long and $710 \mu \mathrm{m}$ in diameter, and the laser entrance hole is $500 \mu \mathrm{m}$ in diameter. The laser beam is $f / 4$ and has its focus $1600 \mu \mathrm{m}$ away from the back wall. A 400 - $\mu \mathrm{m}$-diam spot on the back wall is irradiated at a peak intensity of $1.3 \times 10^{15} \mathrm{~W} / \mathrm{cm}^{2}$.

The Eulerian mesh of the hohlraum where $N_{\mathrm{A}}$ is Avogadro's number, $k$ is Boltzmann's constant, $\mu$ is the mean molecular weight per free particle, $f_{\alpha}$ is the number fraction for species $\alpha$, and $E_{\alpha}$ is the internal energy per particle for species $\alpha$. The term $E_{\alpha}$ is zero for the electrons and is the total ionization energy for the ions. By total ionization energy, we mean (Fig. 4-14) has the $L$ lines running more or less parallel to the $z$ axis, except near the back wall where they bend and then run more or less vertically. The lowest $L=1$ mesh line is the one nearest the $z$ axis, whereas the highest $K=26$ mesh line is the approximately vertical line furthest to the right (it intercepts the z-axis at $z=1172 \mu \mathrm{m})$. Radiation transport was by diffusion with an opacity multiplier of 1 . The diffusion length was limited by a computed geometric mean free path, and this correction was applied to diffusion across both the $K$ and $L$ mesh lines. Gold opacities were obtained from the Cray version of NLTE XSN.

Figure 4-15(a) shows the density contours at the peak of the laser pulse, while Fig. 4-15(b) shows the radiation-temperature contours. The latter were calculated by setting the radiation energy density equal to $a T_{R^{\prime}}^{4}$ where $a=7.565 \times 10^{15} \mathrm{ergs} /\left(\mathrm{cm}^{3} \cdot \mathrm{K}^{4}\right)$ is the Stefan-Boltzmann constant. The radiation temperature reaches $290 \mathrm{eV}$ at the center of the back wall and about $200 \mathrm{eV}$ 


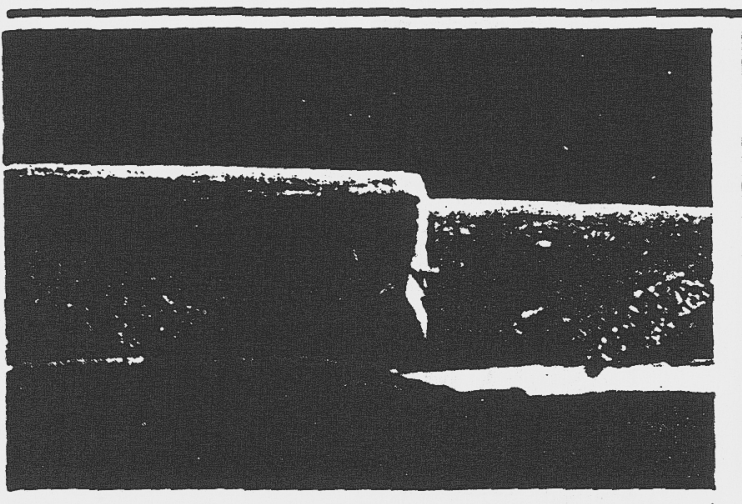

the variations in density were not real, but were only measurement errors. The published density for evaporated aluminum films is $2.70 \mathrm{~g} / \mathrm{cm}^{3}$, which is what has been used in all of our analyses. The shock velocity is given by

$U_{s}^{2}=V_{1}^{2} \frac{P_{2}-P_{1}}{V_{1}-V_{2}}$

where $V_{1}$ and $P_{1}$ are the specific volume and pressure, respectively, in the uncompressed region. For a strong shock, $V_{2} / V_{1}$ will approach some limiting value, which we assume to be independent of $V_{1}$. The velocity of a strong shock is then inversely proportional to the square root of the density. The $1 \sigma$ standard deviation in the density then corresponds to a $4 \% 1 \sigma$ uncertainty in the drive and a $1 \% 1 \sigma$ uncertainty in the radiation temperature.

The other source of error under our control is the calibration of the streak-camera sweep speed. Griffith obtained this sweep speed just after completion of the $2 \omega$ experiments, but before the start of the $4 \omega$ experiments. Changes in the sweep speed during the series have been established as being less than $10 \%$ by comparing the streakcamera fiducial with the incident laser pulse. As additional confirmation, on one shot using etalon structure on the incident laser pulse, the sweep speed was derived to be $112 \mathrm{ps} / \mathrm{mm}$, which compares with the calibration value of $118 \mathrm{ps} / \mathrm{mm}$.

\section{Aluminum Ablation}

Aluminum was chosen as the witness-plate material because it has a well-known equation of state and has a relatively low atomic number, so that its opacity to the ablation front is reasonably low. This low opacity is

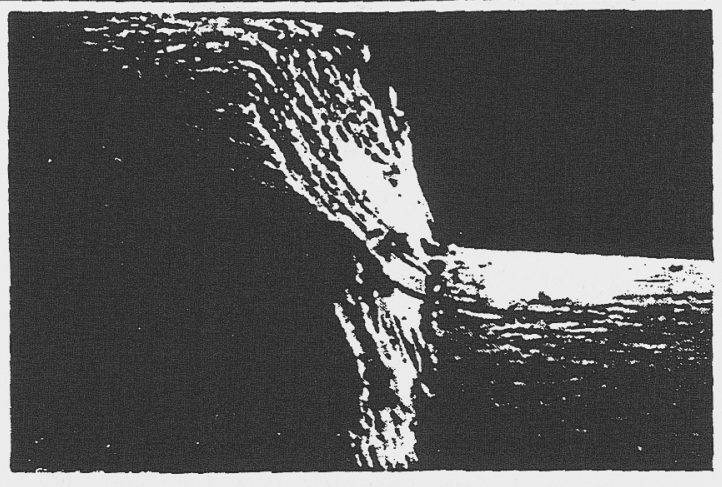

an advantage because, when the opacity is high, the radiation transport is diffusive, and we really have a Marshak wave. The pressure then is no longer simply related to the radiation drive and decreases with time roughly as $1 / \sqrt{t}$. Other low-opacity materials, e.g., beryllium or plastic, were not cho sen because the back surfaces of these materials would be preheated excessively.

Hatchett $^{11}$ has studied the ablation of low $-Z$ materials by soft $x$ rays. Opacity typically is very high in these materials when they are cold, but is low when the materials are ionized. He makes the approximation that the radiation is absorbed at a sharp heat front with a discontinuity in the density, pressure, temperature, and velocity. Two regimes are found, one below a critical temperature (typically a few hundred eV), and one above this critical temperature.

Below the critical temperature, an "expansion heat front" penetrates subsonically into the cold material. Material exits the heat front at exactly the isothermal sonic velocity and goes into an isothermal rarefaction wave. This rarefaction wave is assumed to be kept at the radiation temperature $T_{R}$ by absorbing the energy for its $p d V$ work from the radiation field. To satisfy the conservation conditions for mass, momentum, and energy, the heat front must be preceded by a shock wave. It is this shock wave whose velocity we measure, and it is the pressure behind this shock wave that we call the ablation pressure.

Above the critical temperature, a "compression heat front" penetrates supersonically into the cold material, and no shock wave precedes this front. Material exits supersonically into a compressed uniformflow region that joins with a rarefaction wave. Both the uniform-flow region and the rarefaction wave are at the radiation temperature $T_{R}$.
Fig. 4-13. Scanningelectron-microscope pictures of a bent $43 \mu \mathrm{m} / 67 \mu \mathrm{m}$ aluminum witress plate for a half-Caim target. 
Results of the Novette Implosion Experiments

Only for two 0.8-scale Cairns did Dante A and Dante $H$ simultaneously measure the radiation temperature in both the primary and secondary. One measurement was with a $400-\mu$ m-diam spot at the base of the $480-\mu \mathrm{m}$-diam scattering cone, and the other measurement was with a $680-\mu \mathrm{m}$-diam spot; the latter is closest to the $600-\mu \mathrm{m}$ spot that we used. Dante A looked into the primary and measured 161 to $170 \mathrm{eV}$, while Dante $\mathrm{H}$ looked into the secondary and measured 146 to $149 \mathrm{eV}$. However, Dante A looked obliquely and saw a part of the end cap that was directly irradiated by the laser; this area is expected to have been hotter than the area seen by Dante $\mathrm{H}$ in our experiment on an 0.8-scale Cairn with a witness plate. The tighter focusing in our experiment is expected to increase the secondary temperature, but only by 1 to $2 \mathrm{eV}$.

As far as absolute agreement with Suter's WALLE calculations, the $155-\mathrm{eV}$ radiation temperature inferred from the Dante $\mathrm{H}$ measurement for our experiment comes very close to the WALLE prediction of $151 \mathrm{eV}$. Suter set up WALLE to use Rosen's Marshak scaling, 8 and Suter used the experimental results ${ }^{16}$ for the $x$-ray conversion efficiency of the laser light.

\section{Conclusions}

Radiation drive has been determined indirectly by measuring the velocity of the : shock produced by ablation pressure in aluminum. The accuracy of this technique depends upon the accuracy with which we know the aluminum equation of state, the step height and density of the aluminum witness plate, and the streak-camera sweep speed. The major limitation of the technique is that the preheat must be quite low; otherwise, plasma blowoff from the back surfaces of the steps makes the step height uncertain.

The radiation drive deduced from the shock-velocity technique has been consistently higher than that inferred from Dante measurements, although the latter do not include corrections for hole closure, line of sight, or albedo. Since the Dante measurements were made so recently, it is too early to say how much of this difference can be explained by the corrections. We believe that all the basic measurements are correct.
Both the witness-plate and Dante measurement techniques confirm the Marshak scaling with a higher coupling efficiency [ $\eta$ in Eq. (3)] obtained at 0.53 and $0.27 \mu \mathrm{m}$ than at $1.06 \mu \mathrm{m}$. Both measurement techniques also indicate that the drive is the same for 2630 - and $5270-\AA$ irradiation. According to the witness-plate measurements, a radiation temperature of $210 \mathrm{eV}$ was achieved at $2630 \AA$ with only $0.08 \%$ of the $1.63-\mathrm{kJ}$ laser energy in a $29-\mathrm{keV}$ suprathermal electron distribution, indicating that high-drive, low-preheat hohlraums are attainable.

Author: D. W. Phillion and S. V. Weber

\section{Results of the Novette Implosion Experiments}

\section{Introduction}

The Novette implosion experiments were our first attempt to investigate whether the improvements in hohlraum radiation conditions observed during the Argus wavelength-scaling experiments ${ }^{17}$ at submicron laser wavelengths can significantly enhance the compression of DT fuel capsules. The Argus experiments showed a drastic decrease in electron preheat and a significant increase in thermal $x$-ray conversion efficiency as the laser wavelength . was decreased.

Based on these observations, and on the results of hohlraum and implosion experiments at Shiva, ${ }^{18,19}$ LASNEX preshot calculations ${ }^{20}$ showed that, with $\sim 10 \mathrm{~kJ}$ of $0.53-\mu \mathrm{m}$ laser light in a 1-ns FWHM pulse, compression experiments with current gasfilled DT capsules might achieve fuel densities of $\sim 20 \mathrm{~g} / \mathrm{cm}^{3}$ and temperatures of $\sim 1 \mathrm{keV}$, resulting in fuel pressure of $\sim 1$ to $2 \times 10^{10} \mathrm{~atm}$. Corresponding thermonuclear yields were projected to be $\sim 3 \times 10^{8}$ neutrons. These predictions were conditional on the following assumptions:

- Low electron preheat (less than $10 \%$ of laser energy converted into hot electrons).

- Adequate drive (a radiation temperature of $\sim 160 \mathrm{eV}$ ).

- Adequate drive symmetry. 
Results of the Novette Implosion Experiments

In the Novette implosion experiments described in this article, we used nominal $1-n s$ (FWHM) pulses of $0.53-\mu \mathrm{m}$ light at a nominal total beam energy of $8 \mathrm{~kJ}$ to investigate capsule compression and to obtain hohlraum physics data. Our primary goals were to implode gas-filled capsules in cylindrical hohlraums, to measure capsule performance (compression and neutron yield), and to compare the results with earlier, similar measurements at Shiva with $1.06-\mu \mathrm{m}$ laser light. To achieve these goals, we fired a total of 11 full-energy system shots at Novette.

Our secondary goal was to collect sufficient hohlraum physics data so that we could perform post-series analyses of compression results and could improve our LASNEX semiempirical hohlraum model for both drive and prefreat. We firroc three dedicated shots at Novette (using empty hohlraums) to meet this experimental objective. Additional hohlraum physics data were collected concurrently with compression data during the implosion phase of the series.

\section{Target Description and Irradiation Geometry}

The hohlraums for the Novette implosions were designed in a primary-secondary configuration to provide axisymmetric capsule drive with the two $f / 4$ beams illustrated in Fig. 4-21. In this figure, we show a sketch of a standard 1.0-scale hohlraum used in most of the compression and hohlraum-physics experiments. These targets were $1500-\mu \mathrm{m}$ diam gold or tungsten cylinders, nominally $3440 \mu \mathrm{m}$ long, with rounded ends. Laser entrance holes measured $\sim 725 \mu \mathrm{m}$, approximately one-half the inner diameter of the hohlraum.

The two $45^{\circ}$ half-angle cones shown in Fig. 4-21 divided the hohlraum into primary (in front of the cones) and secondary (between the cones) regions. The cones were supposed to perform three functions: to shield the fuel capsule from direct laser illumination; to scatter unabsorbed laser light into the primary, the secondary, or both; and to emit $x$ radiation that principally would heat the primary. Preshot calculations using the LASNEX and WALLE codes determined that a cone-to-cone separation of $900 \mu \mathrm{m}$ would result in optimal drive symmetry, ${ }^{20}$ assuming geometric optics transport of the laser light.

We intentionally overfilled the cones with light by $\sim 200 \mu \mathrm{m}$ to compensate for beampointing uncertainties. Thus, assuming geometrical optics, approximately $44 \%$ of first-bounce incident laser light missed the cones and ended up directly heating the far ends of the primary regions. The resulting peak irradiance on the cone lateral surface was estimated to be on the order of $5 \times 10^{14} \mathrm{~W} / \mathrm{cm}^{2}$ for the nominal conditions of $4 \mathrm{~kJ}$ of incident $0.53-\mu \mathrm{m}$ laser light.

Most of the capsules used in the compression experiments were nominally 200 $\mu \mathrm{m}$-diam $\times 5$ - $\mu \mathrm{m}$-thick glass shells, each
Fig. 4-21. Laser-target configuration during the Novette implosion campaign.

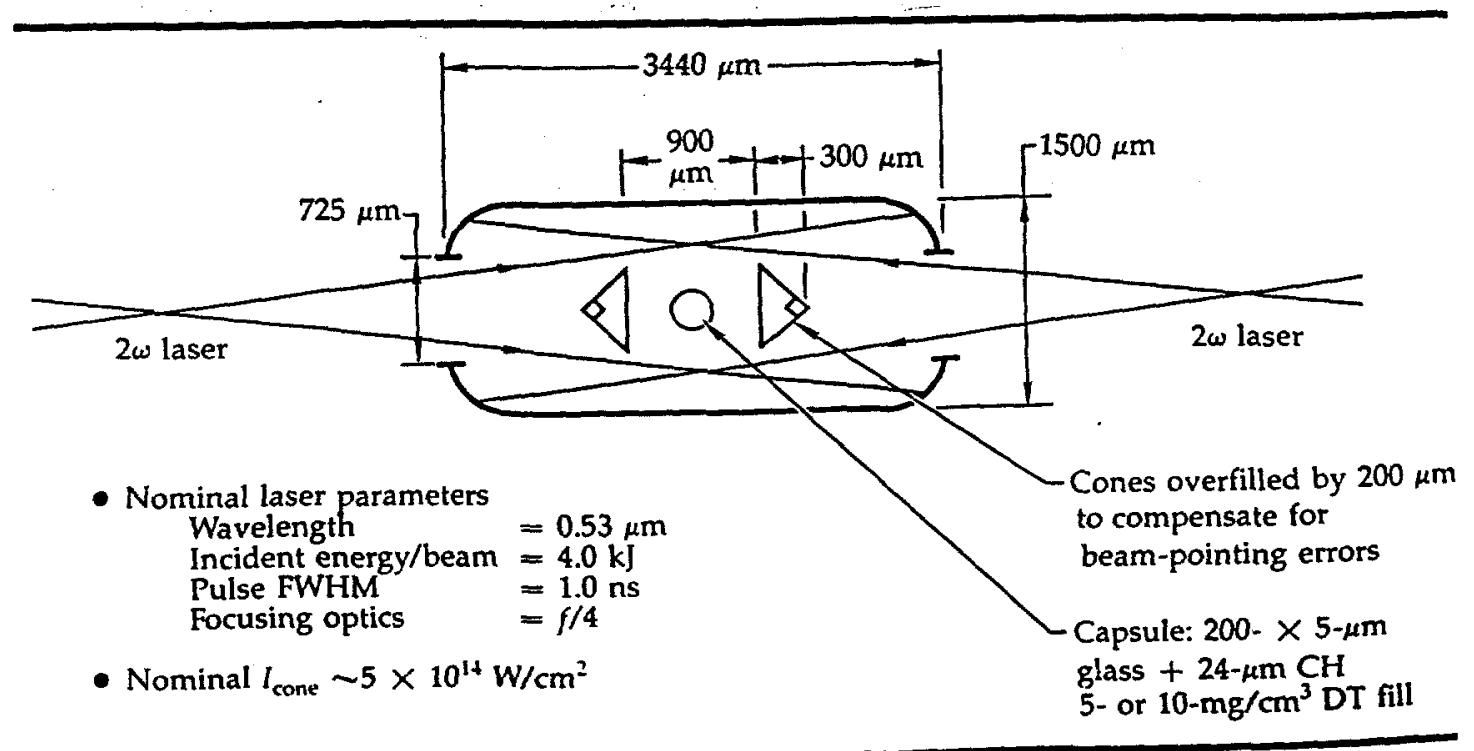


Results of the Novette Implosion Experiments

coated with $24-\mu \mathrm{m}$ of $\mathrm{CH}$ ablator and filled with equimolar gaseous DT at a density of $10 \mathrm{mg} / \mathrm{cm}^{3}$. We also tried to scale neutron yield with the fuel mass by imploding capsules filled with gaseous DT at a density of only $5 \mathrm{mg} / \mathrm{cm}^{3}$. Relative to the capsules containing $10 \mathrm{mg} / \mathrm{cm}^{3}$ of DT, one shot worked well, achieving the highest yield in 1.0-scale hohlraums ( $2 \times 10^{7}$ neutrons). The other shot was a dud, for reasons that we have not yet been able to determine.

Depending on the experiment goals, two general hohlraum types were used to meet our hohlraum-physics diagnostics requirements. The first type, thick-walled gold hohlraums, with $\Delta r=40 \mu \mathrm{m}$, were used for capsule-implosion experiments. We monitored energy deposition along the walls of the hohlraum with a spatially discriminating $x$-ray streak camera (SDSS) viewing a $1-\mu \mathrm{m}$ thick gold patch over a $400-\times 1000-\mu \mathrm{m}$ rectangular hole (see the article, "BurnThrough Foil and Witness Plate Experiments for Novette" in Section 2). For certain shots, we used a different thin foil over a second $400-\mu \mathrm{m}$ hole, through which we tried to image self-emission from the compressed fuel at stagnation with a highresolution $(\Delta x \sim 5 \mu \mathrm{m}) x$-ray pinhole camera. Since the fuel was relatively cold $(<1.0 \mathrm{keV})$, we did not observe any image. During the neutron-activation shots, we used another patch ( $\sim 1000-\mu \mathrm{m}$ diam) mounted on the thick-walled hohlraums and facing the target-debris collector of the neutron-activation diagnostics. The patches over the diagnostics holes minimized perturbation of the radiation symmetry by these holes.

In addition, we experimented with an 0.8 -scale version of the thick-walled gold hohlraums. In the 0.8-scale hohlraum, all linear dimensions were those of the 1.0scale target multiplied by 0.8 . Our primary objective in using the smaller-scale hohlraum was to increase the radiation drive for imploding the capsule.

The second general hohlraum type consisted of thin-walled tungsten targets, with $\Delta r=2 \mu \mathrm{m}$. These thin-walled targets, used to study laser transport and hotelectron energy deposition by means of two-dimensional $x$-ray imaging techniques, also had Dante holes in the primary and secondary for gradient measurements.

\section{Novette Implosion Diagnostics}

A synopsis of the Novette implosion diagnostics is given in Table 4-6. These diagnostics are divided into two generalgroups: hohlraum-physics diagnostics, and compression diagnostics.

Hohlraum Diagnostics. Our hohlraum diagnostics sought to measure radiation drive, temperature distribution in hohlraums, laser-energy transport, and hotelectron preheat. Radiation-drive diagnostics used principally the two Dante 10-channel $X$-ray diode (XRD) systems, Dante $A$ and Dante H (see the article, "Analysis of Dante Data," later in this section) and, to a lesser extent, the witness-plates for radątion-driven shock-velocity measurements (see the preceding article, "RadiationDrive Scaling at $2 \omega$ and $\left.4 \omega^{\prime \prime}\right)$. Data from these techniques were used to infer the radiation temperature.

Energy deposition along the hohlraum wall was measured by the SDSS in a single-channel mode $(\sim 200 \mathrm{eV})$ to obtain the timing of Marshak-wave burn-through by means of the 1- $\mu \mathrm{m}$ foil. The SDSS used a $75-\mu \mathrm{m}$ pinhole in front of a $125-\mu \mathrm{m}$-wide

Table 4-6. Synopsis of Novette implosion diagnostics.

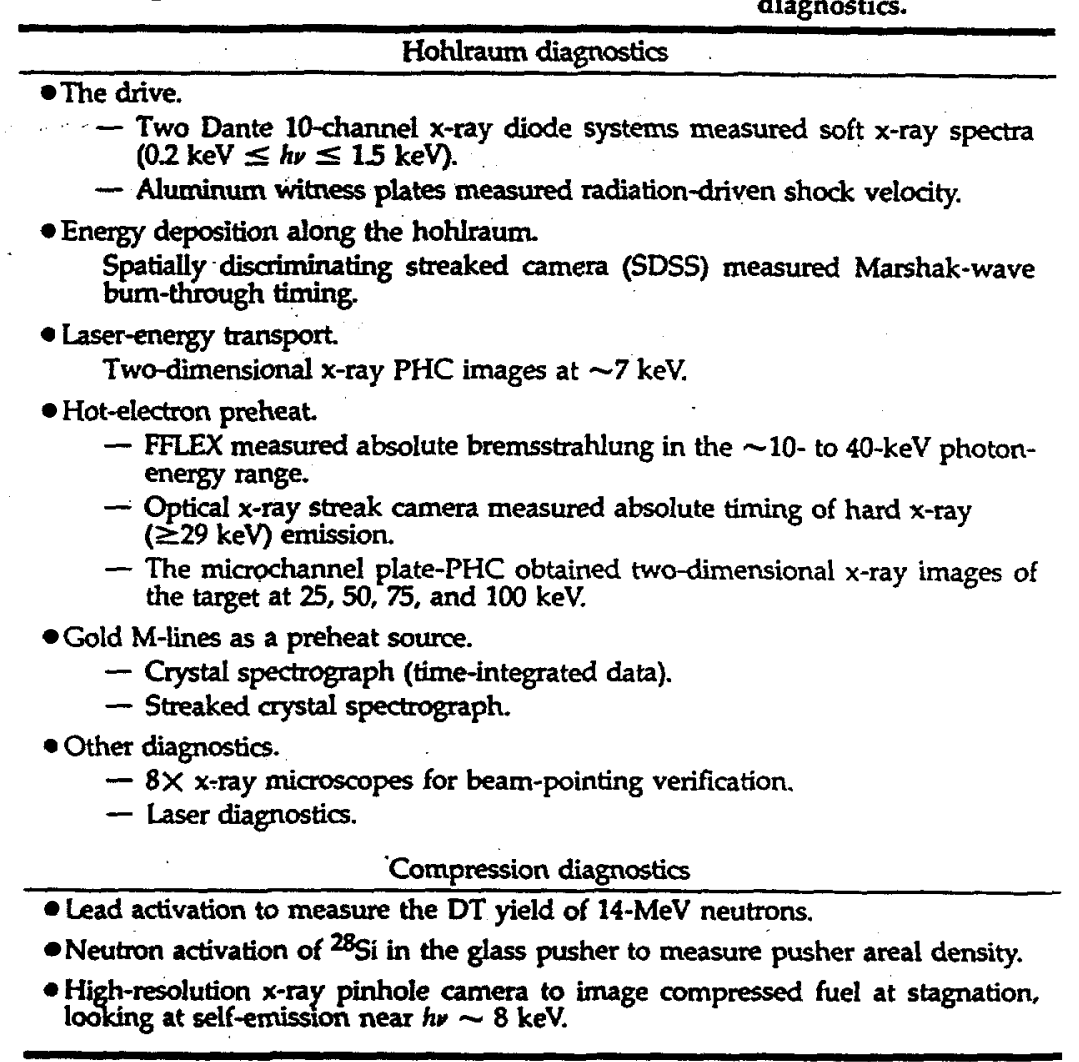


slit to produce a data sampling area of $\sim 1300 \times 125 \mu \mathrm{m}$ at the film plane. The camera had nominal spatial and temporal resolutions of $\sim 80 \mu \mathrm{m}$ and $18 \mathrm{ps}$, respectively. Simultaneous use of the two Dante systems also gave us some crude information about temperature gradients.

Laser-energy transport was measured by taking a time-integrated, two-dimensional $x$-ray image of the thin-walled target with a pinhole camera $(\mathrm{PHC})$ at a photon energy of $\sim 7 \mathrm{keV}$. The camera had four different sizes of pinholes, which provided relative film-exposure information as well as a variable spatial resolution (from $\sim 50$ to $300 \mu \mathrm{m}$ ). These camera characteristics helped us to estimate relative laserenergy deposition at various regions in the troklraum.

Hot-electron preheat levels, timing, and energy deposition were measured by FFLEX, by an optical $x$-ray time-interval streak camera (OX-2), and by a microchannel plate coupled to a PHC.1. The FFLEX gave us absolute bremsstrahlung for the photon energy range of $\sim 10$ to $400 \mathrm{keV}$. From this spectrum, we derived the fraction of the laser energy going into suprathermal electrons $\left(f_{\text {hot }}\right)$ and the temperature of these electrons ( $T_{\text {hot }}$ ).

An OX-2 measured the absolute timing of hard ( $h \nu \geq 29 \mathrm{keV}) x$-ray emission from fast electrons. Finally, a microchannel plate coupled to a PHC obtained twodimensional $x$-ray images of thin-walled targets at photon energies of $25,50,75$, and $100 \mathrm{keV}$. The latter diagnostics provided data on hot-electron energy deposition. These data can help us to estimate the strength of electron preheat in the vicinity of the fuel capsule during compression.

Compression Diagnostics. Compression diagnostics were used to monitor fuelcapsule performance. In particular, we measured DT neutron yield by lead activation ${ }^{22}$ and glass-pusher areal density by neutron activation of the ${ }^{28} \mathrm{Si}$ in the glass. ${ }^{23}$ The latter technique, used during the Shiva implosion experiments, is a very sensitive technique that is well-suited for highcompression, low-fuel-temperature $(Z 500 \mathrm{eV})$ measurements. The fuel density at burn time can be inferred from the pusher areal density using both LASNEX ${ }^{17}$ and a simple analytical model. ${ }^{24}$ The detector efficiency was measured by the $\beta-\gamma$ coincidence counting of the ${ }^{28} \mathrm{Al}$, and the debris collection efficiency was determined using the ${ }^{2+} \mathrm{Na}$ tracer technique. ${ }^{25}$

Major Experimental-Results

Highlights of the experimental results are presented below. We describe measurement results for the radiation drive, hohlraumtemperature distribution, laser-energy transport, electron preheat, and compression. A more detailed account of the Novette implosion experiments can be found in Ref. 26.

Radiation Drive. The Dante XRD systems were our basic drive-monitoring devices. We used them to observe thermal $x$-ray brightness (spectra between $\sim 0.20$ and $1.5 \mathrm{keV}$ ) of both the primary and the secondary, as illustrated in Fig. 4-22. Representative equivalent blackbody temperatures (or radiation temperatures, $T_{\mathrm{R}}$ ) inferred from the data are summarized in the table of Fig.4-22. This table shows that, for 1.0-scale hohlraums, and for laser energies in the range of 8 to $9 \mathrm{~kJ}$, average radiation temperatures in the primary and secondary were $\sim 144$ and $143 \mathrm{eV}$, respectively, making the two regions nearly identical.

Closer examination of the table in Fig. 4-22 shows both some fluctuations and a trend. The fluctuations are evident in both hohlraum scales. Temperatures in the primaries of 1.0-scale targets range from 140 to $151 \mathrm{eV}$, while temperatures in the secondaries of 0.8 -scale targets range from 146 to $160 \mathrm{eV}$. In the next article, "Analysis of the Novette Compression Series," we argue that such fluctuations can be attributed to variations of the experimental details.

The trend is that the 0.8 -scale hohlraums are, not too surprisingly, hotter than the 1.0scale hohiraums. The averaged data show that the 0.8-scale hohlraums appear $\sim 20 \mathrm{eV}$ hotter than the 1.0-scale hohlraums.

We note, however, that the $x$-ray fluxes we measure from a given experiment are not global values that immediately quantify the capsule radiation drive. Rather, these values are local quantities that depend on the details of each particular experiment, e.g., uncertainties in beam pointing, irradiation spot-size setting, absorption of the cones, transport of the unabsorbed light, and differences between the two Dante 
Results of the Novette Implosion Experiments

\begin{tabular}{|c|c|c|c|c|c|c|}
\hline \multirow[t]{3}{*}{ - } & \multirow[t]{3}{*}{$\begin{array}{l}\text { Drive monitoring } \\
\text { in primary }\end{array}$} & \multirow[b]{2}{*}{ Shot No. } & \multirow{2}{*}{$\begin{array}{c}\text { Hohlraum } \\
\text { size } \\
\text { (scale) }\end{array}$} & \multirow{2}{*}{$\begin{array}{l}\text { Laser } \\
\text { energy } \\
\text { (kJ) }\end{array}$} & \multicolumn{2}{|c|}{$\begin{array}{c}T_{\mathrm{r}} \text { from } \\
\text { Dante XRDs }\end{array}$} \\
\hline & & & & & $\begin{array}{c}\text { Primary } \\
(\mathrm{eV})\end{array}$ & $\begin{array}{c}\text { Secondary } \\
\text { (eV) }\end{array}$ \\
\hline & & 93100303 & 1.0 & 8.53 & - & $140(A)$ \\
\hline & & 93101105 & 1.0 & 9.00 & $140(A)$ & $1+8(\mathrm{H})$ \\
\hline $2 \omega$ laser & & 93101313 & 1.0 & 9.00 & 151 (A) & - \\
\hline & & 93102615 & 1.0 & 8.15 & $140(\mathrm{H})$ & $140(A)$ \\
\hline & & 93120807 & 1.0 & 7.79 & $133(\mathrm{H})$ & - \\
\hline & $\nabla$ & 93091503 & 0.8 & 8.40 & $161(A)$ & $146(\mathrm{H})$ \\
\hline - & $\begin{array}{l}\text { Drive monitoring } \\
\text { in secondary }\end{array}$ & 93110109 & 0.8 & 6.71 & $163(\mathrm{~A})$ & $160(H)$ \\
\hline
\end{tabular}

systems. Such details can be used to explain the $\sim 13 \%$ difference between the measured secondary radiation temperatures $(140 \mathrm{eV})$ and our preshot capsule drive estimate $(160 \mathrm{eV})$, although other effects such as hole closure 27,28 and plasma density and temperature profiles ${ }^{29}$ sould also have affected the Dante results.

The point here is that, although our projection of a $160 \mathrm{eV}$ capsule drive temperature in the 1.0-scale targets assumed a laser energy $9 \leqslant E \leqslant 11 \mathrm{~kJ}$, the actual energy on target was in the range of 7 to $9 \mathrm{~kJ}$. On the basis of Marshak scaling, this lower energy range would decrease the temperature by only $7 \%$, since the radiation temperature is related to the laser energy and to other laser-target coupling parameters ${ }^{30}$ by

$T_{\mathrm{r}}=\left[\frac{\left\{(\mathrm{CE})\left(\text { total ABS) } E_{\mathrm{L}}\right.\right.}{0.44 \tau_{\mathrm{L}}^{0.57} A_{\mathrm{w}}}\right]^{1 / 3.05}$

where $C E=x$-ray conversion efficiency, total $A B S=$ total fraction of laser energy absorbed by the target, $E_{\mathrm{L}}=$ laser energy (in hJ), $\tau_{\mathrm{L}}=$ laser pulse width (in ns), and $A_{\mathrm{w}}$ = surface area of hohlraum inner wall (in $\mathrm{mm})$. Assuming a total absorption of $\sim 80 \%$ (as suggested by our energy-balance - diagnostics), and an x-ray conversion efficiency of $50 \%$ at the $0.53-\mu \mathrm{m}$ laser wavelength, we believe it is energetically possible to have a 1.0 -scale hohlraum at $\sim 155 \mathrm{eV}$ with only $8 \mathrm{~kJ}$ of laser energy on target. It is possible that the capsule drive temperatures in our hohlraums were higher than the Dante measurements indicate.

Motivated in part by these considerations, we used a witness-plate (a stepped aluminum slab covering a radiation hole in the hohlraum wall) to infer the radiation temperature in the secondary of an 0.8-scale target (shot No. 93121905). The witnessplate was used to measure the radiationdriven shock velocity, from which we inferrred the radiation temperature (see the preceding article, "Radiation-Drive Scaling at $2 \omega$ and $\left.4 \omega^{\prime \prime}\right)$. In that experiment, we also used Dante $\mathrm{H}$ to monitor the temperature in the primary region of the same target. The results were that, at an incident laser energy of $7.2 \mathrm{~kJ}$, the secondary radiation temperature inferred from the shockvelocity measurements was $172 \pm 5 \mathrm{eV}$, which was $17 \pm 5 \mathrm{eV}$ higher than the primary temperature inferred from the Dante $(155 \mathrm{eV})$.

We can.explain the 17-eV discrepancy with two considerations. First are the details of the experiment, as discussed above. We should not expect the capsule drive temperature to be the same as a local primary temperature. When we try to estimate the effects of the experimental details with our semiempirical hohlraum model (see the following article, "Analysis of the Novette Compression Series"), we find that we might expect about a 5-eV difference between the primary Dante temperature and the flux onto the witness-plate. Second, we can add another $10 \mathrm{eV}$ to the Dante $\mathrm{H}$ temperature to acount for our systematic neglect of hole closure. ${ }^{27.28}$ With these corrections, we have good agreement between the Dante and the witness-plate values.

In summary, we believe that the temperatures we infer from Dante are local values that depend both on the details of the experiment, such as beam pointing and spot size, and on the gross features of the experiment, such as laser energy and scale size. The scaling trend shown by these local values is that 0.8-scale hohlraums are about $20 \mathrm{eV}$ hotter than 1.0-scale hohlraums. In the following article, "Analysis of the Novette Compression Series," we use the
Fig. 4-22. Dante drive measurements and representative results for 0.8 - and 1.0 -scale hohlraums. 
Dante data to check our semiempirical hohlraum model, which we developed from other data.

Hohlraum-Temperature Distribution. We used the SDSS to monitor energydeposition gradients along the hohlraums, and the results of these measurements are summarized in Fig. 4-23. In this figure, we plot relative delays of the radiation burnthrough signal, as seen by the SDSS looking at the $1-\mu \mathrm{m}$ foil, vs axial positions in the hohlraum. Earliest burn-through signals t... originate from the target region over the cone. Away from the cone region, the signal delays increase to $\sim 400 \mathrm{ps}$ at the primary and to $\sim 250 \mathrm{ps}$ at the secondary. The data shown in Fig. 4-23 infer that the energy flux ( $x$ ray and laser light) on the wall was most intense near the cones, resulting in faster bum-through times, and was reduced in both the primary and secondary regions. The flux intensity was somewhat less in the primary region than in the secondary region.
Fig. 4-23. Plot of relative delays of radiation burn-through signals vs axial positions in the hohlraum
These temperature-distribution data show the nonuniformity of the hohlraum environment. Inference of quantitative energyflux gradients from this type of data is dependent not only on the relative mix of laser intensity and $x$-ray radiation intensity but also on the opäcity model that we use.

Laser-Energy Transport. We obtained our information on laser-energy transport from an x-ray pinhole camera. This camera was configured to record a two-dimensional $x$-ray image of a thin-walled target at a photon-energy level of $\sim 7 \mathrm{keV}$. Representative results from this diagnostics system are shown in Fig. 4-24 for a 1.0-scale target at a total laser energy of $9.0 \mathrm{~kJ}$. Two film images of equidensity contours that were obtained at different radial positions during the same shot are shown on the left side-of the figure.

We studied laser-energy deposition by examining contrasts in film density along the total image length of the different radial positions. Two lineouts taken along the image length are shown on the right side of Fig. 4-24.

From Fig. 4-24, we see that cone emission was much higher than wall emission, implying that more laser energy per unit area was deposited on the cones than on the hohlraum wall (the right-hand cone emission is saturated). Although the camera was not absolutely calibrated, we can get some idea of the relative exposure between the cones and the rest of the target by comparing images from the different sizes of pinholes (film exposure is proportional to the square of the pinhole diameter). Our estimate, based on all the data taken, is that the exposure at the cones was on the order of 35 times the exposure from the rest of the target. In the next article, "Analysis of the Novette Compression Series," we use this estimate to set an upper bound on the laser intensity striking various parts of the hohlraums.

From the bottom lineout in Fig. 4-24, we see that some laser-beam energy was deposited on the hohlraum exterior as a consequence of beam-pointing errors. Such incidents probably would create problems of drive asymmetry.

Electron Preheat. Our hot-electron diagnostics consisted of the FFLEX for absolute hard $x$-ray levels, the OX-2 for absolute hard $x$-ray emission timing, and the 
Results of the Novette Implosion Experiments
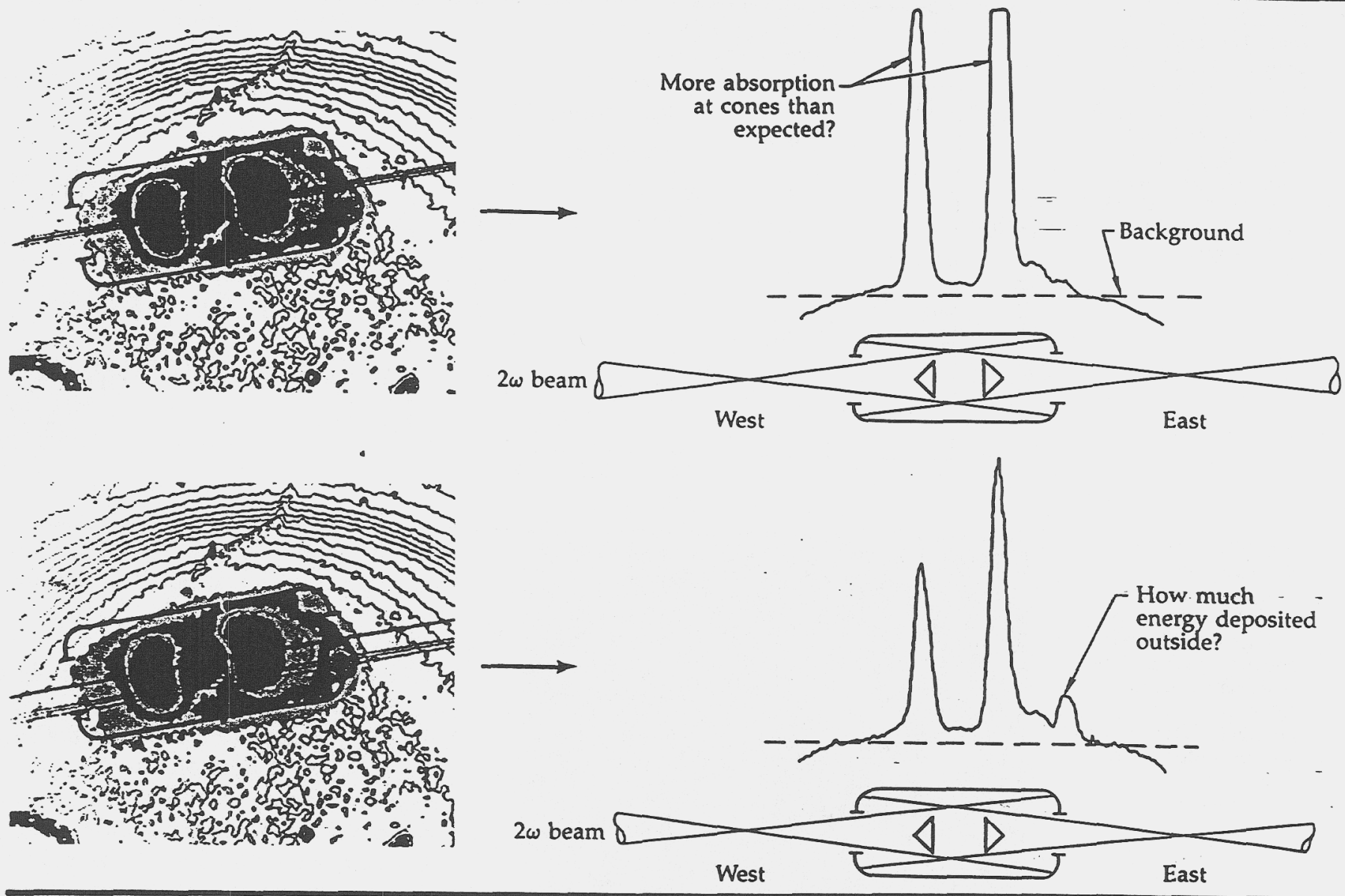

microchannel plate-PHC combination for time-integrated hot-electron energy deposition. We first summarize the results for absolute preheat levels and timing and then describe the results for hot-electron energy deposition.

Representative results from the FFLEX and the OX-2 are shown in Fig. 4-25 for an 0.8-scale hohlraum. In Fig. 4-25(a), we plot hard $x$-ray fluences vs the photon energy from absolute bremsstrahlung measurements with the FFLEX. The fluences are relatively low for a multikilojoule hohlraum experiment. ${ }^{17}$ We inferred $f_{\text {hot }}$ and found it to be much less than $1 \%$ for a 1.0 -scale hohlraum and $\sim 2 \%$ for an 0.8-scale hohlraum. Both values are much lower than the upper limit we used in our preshot calculations $(\sim 10 \%)$. The relatively higher electron preheat at 0.8 scale is evidence for plasmafilling phenomena in relatively smaller hohlraums. ${ }^{1,10}$ However, temperatures of fast electrons were typically on the order of $30 \mathrm{keV}$ for both target sizes. We note that attenuation in the $\sim 40-\mu \mathrm{m}$ wall thickness may have affected data corresponding to the $\leq 50-\mathrm{keV}$ photons, introducing significant uncertainties in that part of the measured spectrum.

In Fig. 4-25(b), we show data for timeresolved hard $x$-rays ( $h \nu \geq 29 \mathrm{keV}$ ) from the $O X-2$. The figure shows the timing of the $x$-ray emission relative to the laser pulse, and we see that the hard $x$-ray signal trails the laser pulse by $\sim 400 \mathrm{ps}$ for the 0.8 -scale hohlraum. Similar $x$-ray signals for a 1.0-scale target show the $x$-ray signal trailing the laser pulse by $\sim 600 \mathrm{ps}$. In either case, our hohlraum physics data show low levels of electron preheat that were delayed relative to the laser pulse and, by inference, to the drive (no such delays were observed in planar target experiments). ${ }^{26}$ We conclude that electron preheat did not play a deleterious role during the Novette compression experiments.

We studied time-integrated hard $x$-ray emissions with the microchannel plate-PHC combination to collect information on electron preheat distribution in a hohlraum. ${ }^{21}$ The results of these studies are represented in Fig. 4-26 for an 0.8-scale thin-wall hohlraum. Photon energies are also given for the four images shown. The data suggest
Fig. 4-24. Representative results of energy-transport diagnostics from the $7-\mathrm{keV} x$-ray pinhole camera. 
Fig. 4-25. (a) Representative results from absolute bremsstrahlung measurements with FFLEX for an 0.8scale target. (b) Absolutely time-resolved hard $x$-ray emission from the OX-2 streak camera for an 0.8 scale target.

Fig. 4-26. Two dimensional $x$-ray images for suprathermal-electron energy-deposition information for an 0.8scale target. Data taken by a microchannel plate coupled to a pinhole camera.
Results of the Novette Implosion Experiments
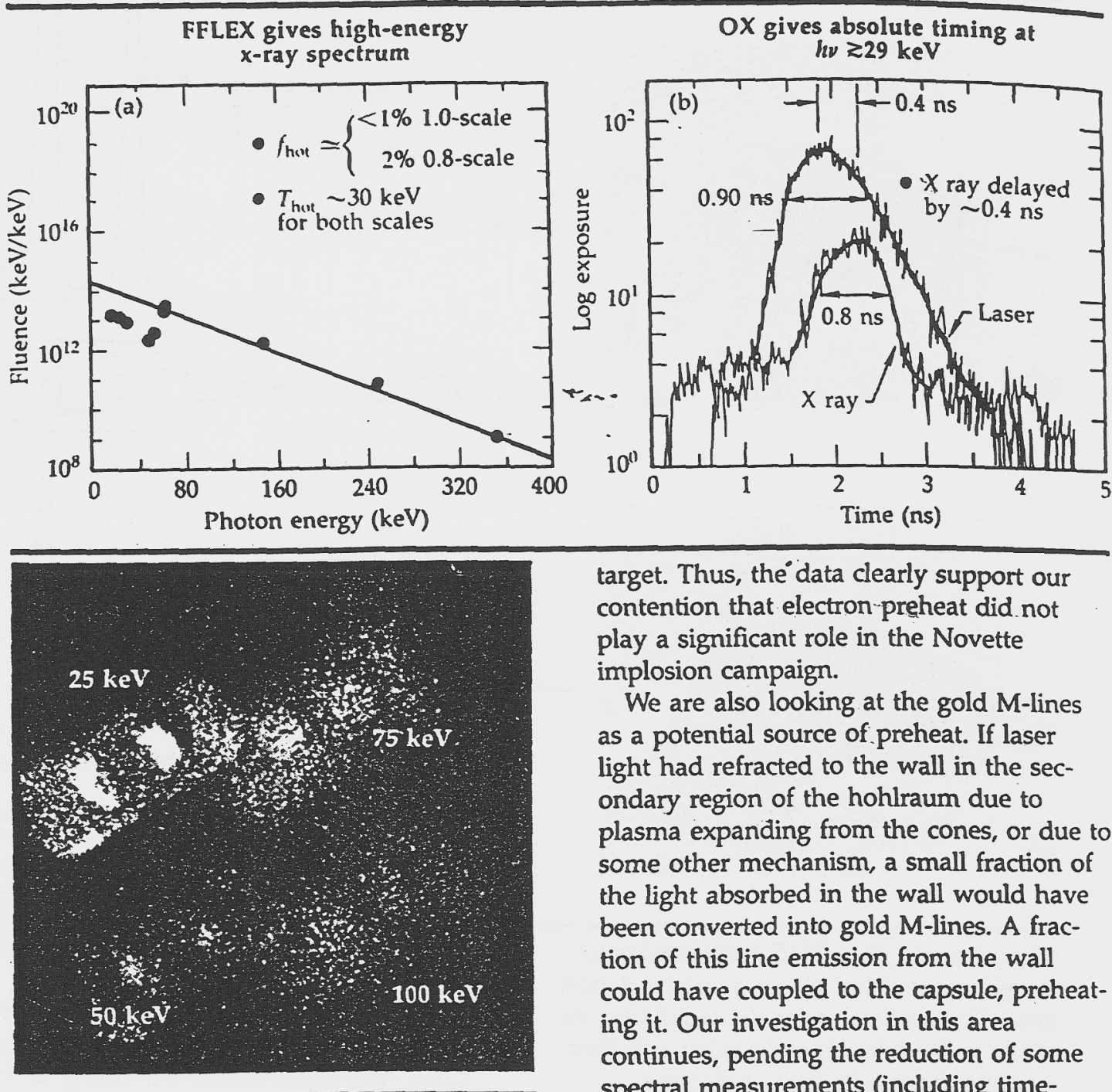

that the most intense hot-electron energy deposition per unit area took place at the cones. Although some energy was deposited in the primary, very little energy per unit area was deposited in the secondary. (We continue our efforts to quantify these differences in deposition through detailed film analysis; final results will appear in Ref. 26.) The qualitative features of the data suggest that only marginal levels of electron preheat existed in the region where the DT fuel capsule was located. Furthermore, when we used a similar diagnostics technique to monitor hot-electron deposition in 1.0 -scale hohiraums (which were used in most of our compression experiments on gas-filled targets), the $25-\mathrm{keV}$ image was qualitatively similar to those shown in Fig. 4-26, although energy deposition per unit area at the hohlraum wall seemed to be somewhat lower than for an 0.8-scale target. Thus, the data clearly support our contention that electron preheat did not play a significant role in the Novette implosion campaign.

We are also looking at the gold M-lines as a potential source of preheat. If laser light had refracted to the wall in the secondary region of the hohlraum due to some other mechanism, a small fraction of the light absorbed in the wall would have been converted into gold M-lines. A fraction of this line emission from the wall could have coupled to the capsule, preheating it. Our investigation in this area continues, pending the reduction of some spectral measurements (including timeresolved data) that we made during the implosion series. data from neutron-activation studies. Collected data include the $14-\mathrm{MeV}$ neutron yield and the areal density $(\rho \Delta r)$ of the glass pusher at bum time. The neutron yield served as a monitor of the mass-averaged fuel-ion temperature, while the areal density measured the compression of the pusher density (or areal density). ${ }^{18-20,22,23}$

In this short article, we discuss only the data collected when the neutron yields and pusher areal densities were measured simultaneously. For a presentation of all the results from our compression measurements, please see Ref. 26.

Figure 4-27 summarizes the results of our implosion experiments that employed neutron activation of ${ }^{28} \mathrm{Si}$. In this figure, we plasma expanding from the cones, or due to

Compression. We obtained compression and can be used to infer the imploded-fuel 
Results of the Novette Implosion Experiments

have plotted the ratio of the pusher areal density at burn time, $(\rho \Delta r)_{\Gamma}^{\mathrm{B}}$, to the initial areal density, $(\rho \Delta r)_{\Gamma}^{0}$, vs the corresponding neutron yield. The data include representative results for gas-filled capsules in both 0.8 - and 1.0-scale hohlraums.

We have included in Fig. 4-27 the best $\lambda$-ray-driven compression results from Shiva experiments at $1.06-\mu \mathrm{m}$ laser wavelength so that the Novette data can be compared with these earlier multikilojoule compression data. From the figure, we see that Novette targets reached much higher pusher areal-density ratios than Shiva targets with comparable neutron yields $\left(\sim 10^{7}\right)$. ive also see that the average pusher arealdensity ratio (including both $0.8-$ and $1.0-$ scale targets) for Novette targets is about 54, approximately three times the corresponding average from the best Shiva, $1.06-\mu \mathrm{m}$ experiments.

We used a simple analytical model ${ }^{24}$ to estimate the average fuel density achieved in these Novette compression experiments. From this model (which, in the version used here, does not include the effects of pusher-fuel mix), we inferred an average fuel density of $\sim 120 \times$ liquid DT density. ${ }^{26}$

Results using hydrodynamic computer simulations (see the following article, "Analysis of the Novette Compression Series," later in this section) confirm that the $0.53-\mu \mathrm{m}$ Novette data are consistent with $100 \times$-class performance. Our results thus demonstrate the effectiveness of a submicron-wavelength laser in compressing $x$-ray-driven DT capsules to densities higher than could be obtained at Shiva with 1.06- $\mu \mathrm{m}$ light.

Preshot calculations indicated that the fuel temperature for Novette targets would be on the order of $1.0 \mathrm{keV}$; however, fuel temperatures inferred from the data are only in the $\sim 500-$ to $600-\mathrm{eV}$ range. We believe that inadequate symmetry and pusherfuel mix are among the most likely factors that may have degraded the fuel temperatures, thus limiting the neutron yield to, at most, $2 \times 10^{7}$.

Further examination of Fig. 4-27 shows that, although implosions in 1.0-scale hoh!raums achieved higher compressions than implosions in 0.8-scale hohlraums, neutron yields were basically the same for both types of targets. At first glance, these

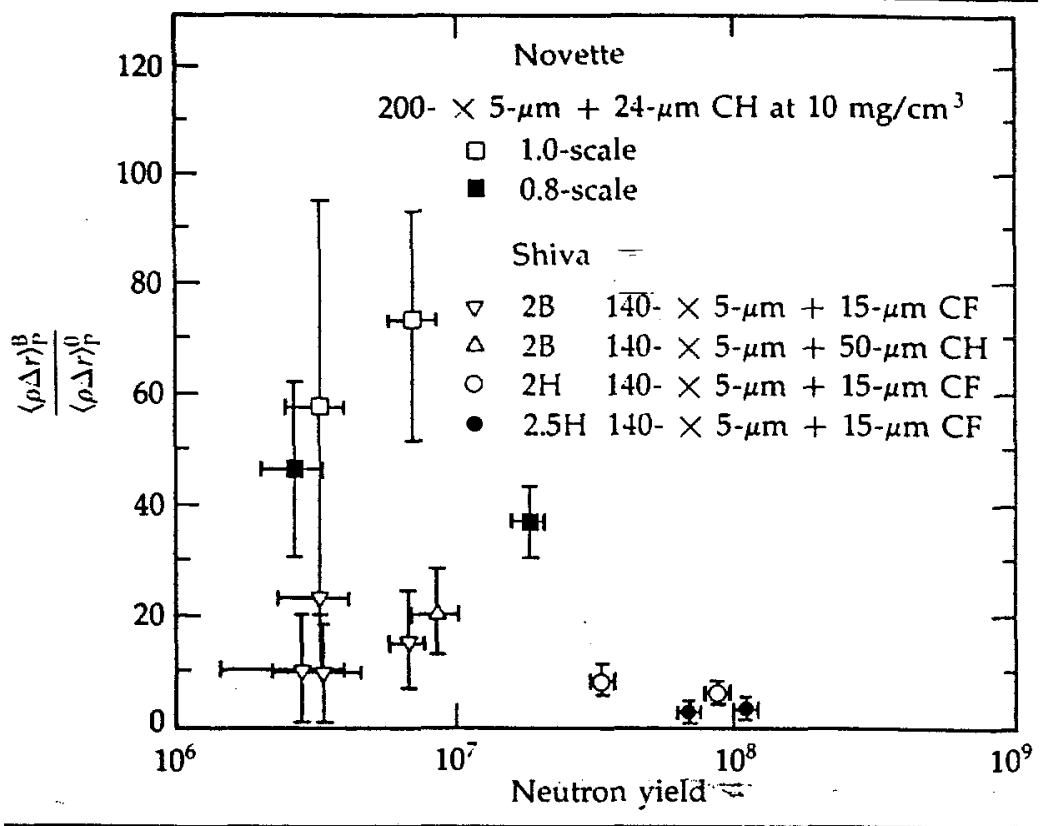

puzzling results tended to suggest that the neutron yields are not very sensitive to changes in the drive levels $(0.8$-scale hohlraums were found to be $\sim 20 \mathrm{eV}$ hotter than 1.0-scale hohlraums). Such an inference would lack credibility, however, because we do not have data on either drive symmetry or pusher-fuel mix. Furthermore, the neutron-yield data for 0.8 -scale targets show enough scatter to suggest that experimental uncertainties (e.g., errors in beam pointing, errors in spot-size setting, and other unknown factors) may have affected the results.

\section{Summary}

Our hohlraum-physics data showed:

- A low preheat $\left(f_{\text {hot }}\right)$ of $<1 \%$ for 1.0 -scale hohlraums and $\sim 2 \%$ for 0.8 -scale hohlraums.

- Moderate radiation temperatures of $\sim 140 \mathrm{eV}$ for primaries and secondaries in 1.0 -scale targets and $\sim 160 \mathrm{eV}$ for primaries and secondaries in 0.8-scale targets.

- Energy-deposition gradients along the hohlraum.

Our implosion-diagnostics data showed:

- Good compression of gas-filled capsules, with an average pusher $(\rho \Delta r)_{\mathrm{p}}^{\mathrm{B}} /\left\langle_{\rho} \Delta r\right\rangle_{\mathrm{p}}^{0} \sim 54$ and an inferred fuel density $\sim 120 \times$ liquid DT density.

- Both neutron yield and fuel temperature were lower than expected, with the highest
Fig. 4-27. Summary of Novette compression results and representative data from best $x$-ray-driven compression results of Shiva. 
Analysis of the Novette Compression Series

neutron yield being $\sim 2 \times 10^{7}$ and the inferred fuel temperature being $\sim 550 \mathrm{eV}$.

- No direct information on implosion symmetry.

\section{Author: F. Ze}

Major Contributors: E. M. Campbell,

R. L. Kauffman, D. W. Phillion, and

L. J. Suter

\section{Analysis of the Novette Compression Series}

\section{Introduction}

We have used a recently developed semiempirical hohlraum model to analyze capsule implosions in the Novette compression series. This model provides us with a more detailed understanding of our hohlraums than was possible in previous experimental series and allows us to make an educated guess about the total radiation flux and the symmetry of the flux that strikes the capsules. On the basis of our modeling, we now believe that these capsule implosions suffered from poor drive symmetry in the polar direction and that this poor symmetry may have been a major cause of the relatively low neutron yields we observed in 0.8-scale hohlraums.

Our semiempirical model is based on support experiments, add-on hohiraum diagnostics, and numerical simulations. This article describes the model and shows how it is consistent with other hohlraum diagnostics.

\section{Pieces of the Model}

Figure 4-28 shows the three basic experimental pieces that we assembled into a semiempirical model: laser-cone interactions; conversion of laser energy, $E_{L}$, to radiation energy, $E_{\mathrm{rad}}$; and our long experience with scaling the wall heating and reemission in hohlraums. For the first piece of our model, we shot several bare cones in primary-secondary hohlraums so that we could estimate both the absorption of laser light by the scattering cones and the distribution of the unabsorbed (scattered) light.
Fig. 4-28. Three basic experimental parts from which our semiempirical hohlraum model was constructed.

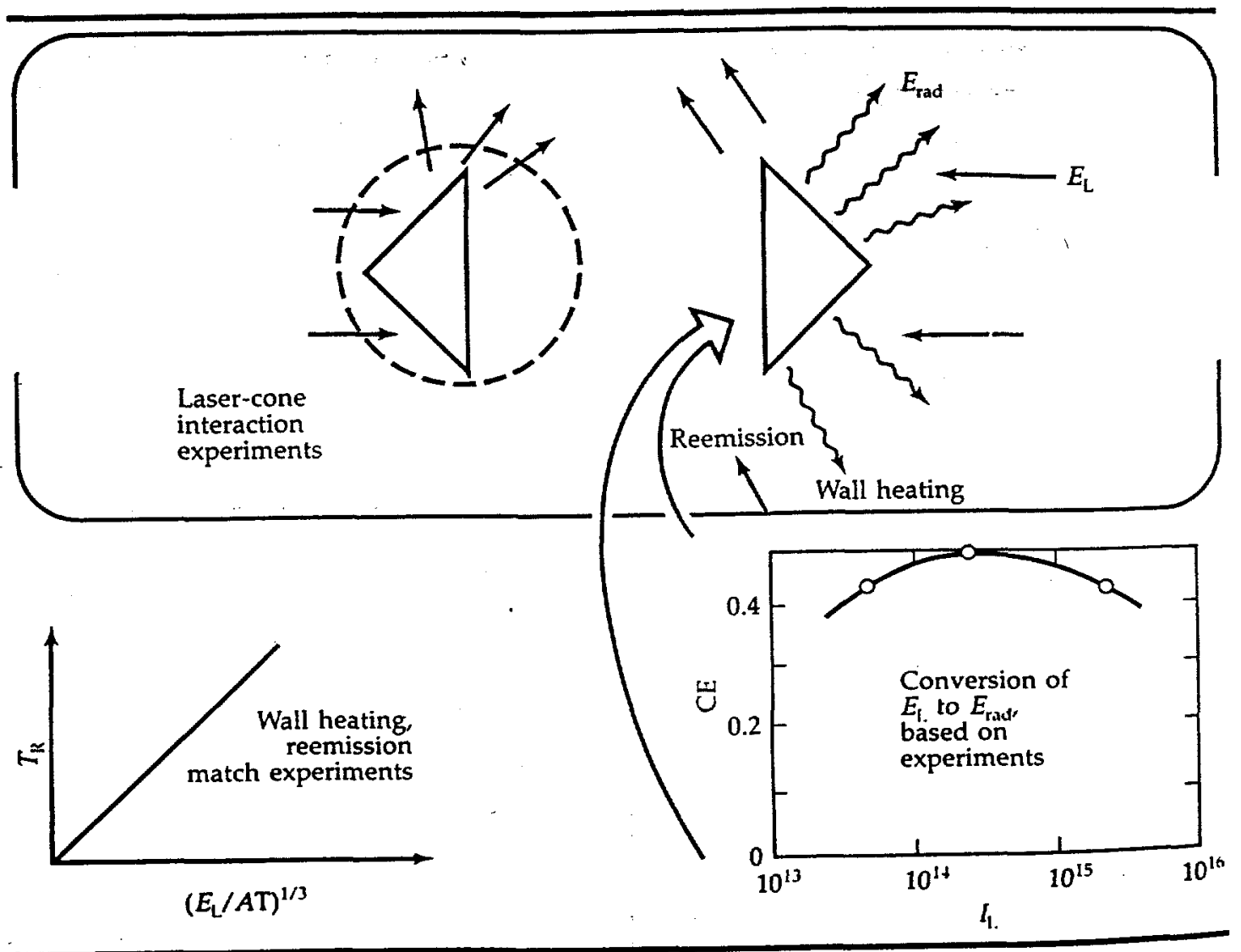


Analysis of the Novette Compression Series

Dante data from these experiments indicate that the cones absorb laser light very heavily ( $\sim 70 \%$ absorption), that the absorbed laser light is converted to sub-keV $x$ rays with a conversion efficiency of 30 to $45 \%$, and that the cones scatter unabsorbed light into the secondary (see the following article, "Analysis of Dante Data"). The polar diagram shown in Fig. 4-29 shows our inferred distribution of scattered light, the most important part of which is the component coming off the face of the cone and heading into the secondary. Note that we really have less information about the scattered light than the diagram implies; the distribution shown in the diagram results from dividing a sphere into six angular regions, measuring the amount of light scattered into each region, and then uniformly distributing that energy over each region.

The second empirical part of our model comprises the intensity-dependent conversion efficiencies that we measured earlier on the Argus laser. ${ }^{31}$ We use these conversion efficiencies to estimate the amount of radiation generated when laser light is absorbed on the various surfaces of the hohlraum.

The third empirical part of our model is our long experience in hohlraum-scaling experiments. On the basis of this experience, we assume that the theory that explains the wall heating and reemission in previous experiments also accurately describes the way in which laser-produced radiation heats the hohlraum walls. ${ }^{31,32}$

\section{Assembling the Pieces}

We use the WALLE code ${ }^{33}$ to assemble the . experimental pieces into a semiempirical model. WALLE allows us to divide our hohlraum into a large number of surfaces and to specify our opinion of radiation production (absorbed laser intensity times conversion efficiency) on those surfaces that we think are directly illuminated.

Our hohlraum-scaling experience enters the modeling through the gold equation of state. WALLE calculates how much flux each surface in the problem receives from every other surface and uses the flux, together with the equation of state, to estimate how the surface is heated. The equation of state has been tuned, with the aid of

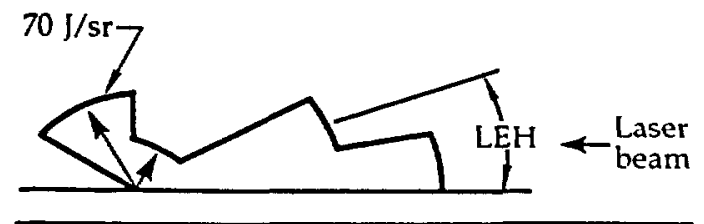

Fig. 4-29. Polar diagram of scattered-light distribution, inferred from the cone experiments that we use in our hohlraum model.

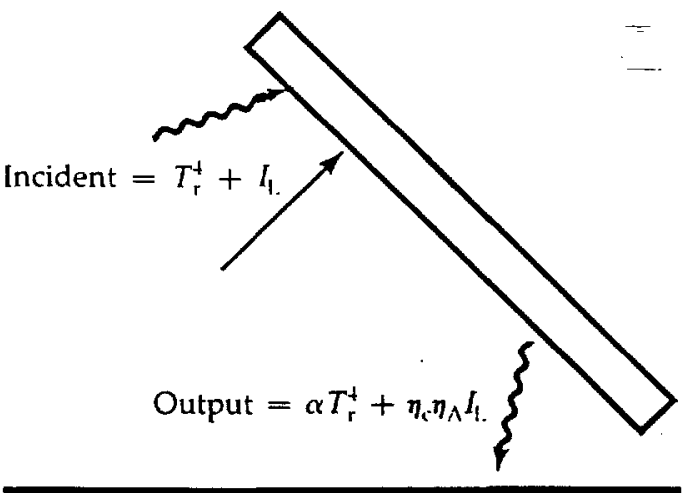

auxiliary calculations, to match scaling in one-dimensional-like situations. Oưr main concern about this procedure is that our calculated hohlraum temperatures may be coming out $\sim 8 \mathrm{eV}$ cooler than they actually are because scaling theory and experiments do not include corrections for hole closure and instrument response time (see the following article, "Analysis of Dante Data").

Our version of WALLE allows us to model gold that is heated by both laser light and radiation. ${ }^{34}$ Figure 4-30 shows such a surface. When a combination of laser light and radiation is incident upon the surface, the radiation flux out of the surface is

Flux $=\alpha T_{\mathrm{r}}^{4}+\eta_{\mathrm{ce}} \eta_{\mathrm{abs}} I_{\mathrm{L}}$,

where $\alpha$ is the albedo of the surface, Our semiempirical model assumes that this albedo is not changed when a surface is heated by laser light.

Our semiempirical hohlraum model gives us estimated values both for laser and radiation fluxes onto all surfaces and for radiation fluxes away from all surfaces. Figure 4-31 shows how we model a 1.0-scale hohlraum. The two laser beams overfill the $600-\mu \mathrm{m}$ scattering cones by $200 \mu \mathrm{m}$. In our nominal model, we absorb $70 \%$ of the geometric fraction of light that strikes the cones and convert $40 \%$ of the absorbed light into radiation. The fraction of light that strikes the cones but is not absorbed $(30 \%)$ is distributed according to the polar diagram of Fig. 4-29. This scattered light produces an intensity distribution along the primarysecondary wall that peaks at about
Fig. 4-30. WALLE allows us to model the emission from surfaces that are heated by both laser radiation and $x$ rays. 
Fig. 4-31. Estimate made by our model of the laser and $x$-ray fluxes in a 1.0-scale hohlraum.

\section{Analysis of the Novette Compression Series}

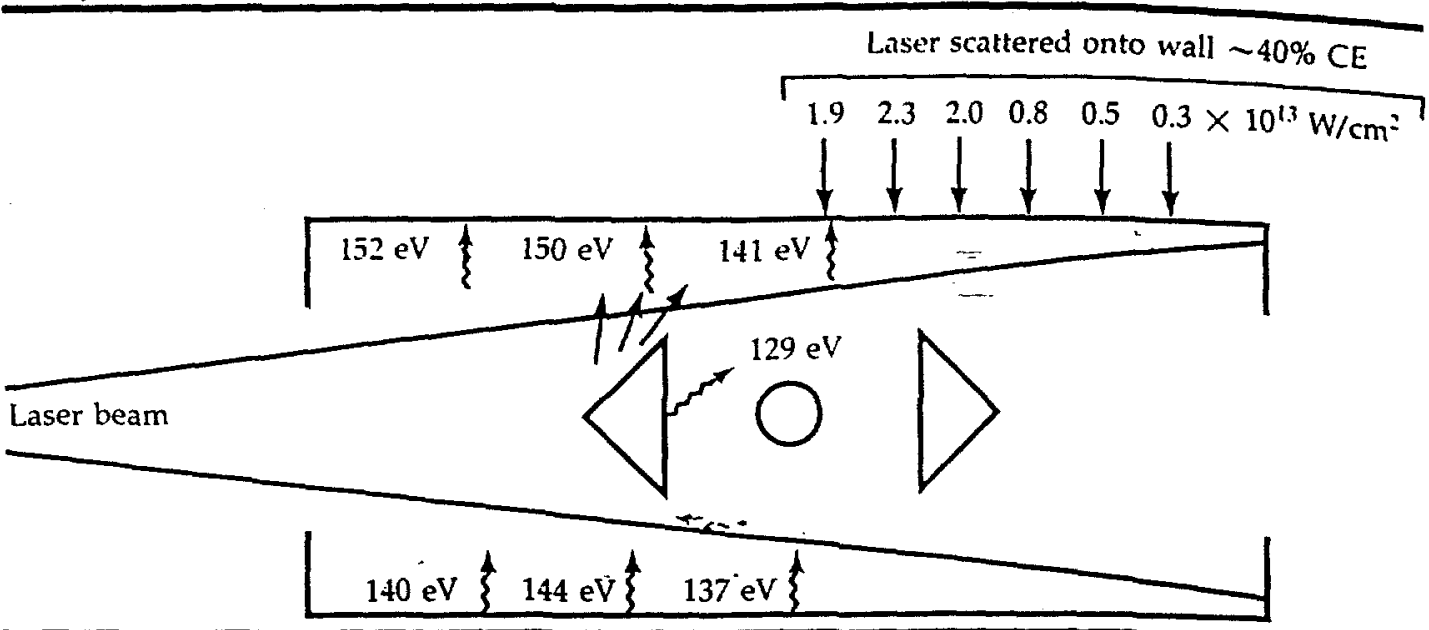

$2 \times 10^{13} \mathrm{~W} / \mathrm{cm}^{2}$ in the secondary. We assume that the secondary light is converted into radiation with a $40 \%$ conversion efficiency.

Our modeling assumes that cone absorption, conversion efficiency, and scatteredlight distribution are all axisymmetric. However, we have made no measurements that test this assumption.

The fraction of light that misses the cones continues down to the endcaps, where it is totally absorbed with a $45 \%$ conversion efficiency. Geometric optics would produce an intensity of $\sim 3 \times 10^{1+} \mathrm{W} / \mathrm{cm}^{2}$ on the endcaps; however, we spread the energy over a larger-than-geometric area, giving an intensity of $\sim 1 \times 10^{14} \mathrm{~W} / \mathrm{cm}^{2}$.

In the real experiment, the deposition on the endcaps would not be axisymmetric because the beams are not perfectly aligned. (That is why we overfill the cones in the first phase: to compensate for the beam misalignment.) Our modeling indicates that asymmetric deposition of light on the endcaps does not directly result in significant azimuthal asymmetry at the capsule because the capsule does not see endcap emission. By the time the radiation generated on the endcaps finds its way to the capsule, the radiation has been azimuthally symmetrized by the hohlraum.

Figure 4-31 also shows estimates of radiation fluxes given by our model. At the top of the figure are radiation fluxes into the wall; at the bottom are fluxes out of the wall, including the contribution due to laser-produced radiation. Because laser energy gets into the secondary, the capsule sees hotter emission from the secondary walls than it does from the back of the cones. This difference in emissions gives rise to a $P_{2}$ drive asymmetry, which is discưssed later in this article.

\section{Checking our Model}

We check our model by comparing model predictions with actual measurements. Later in this article, we demonstrate reasonably good agreement between our model and these diagnostics. Note, however, that this good agreement is only a necessary requirement for our model to be correct; the agreement is not sufficient proof of correctness. One area of concern is that none of the diagnostics check our assumption of axisymmetric laser-cone interactions. In addition, other modeling situations that we have not yet investigated might also match the diagnostics.

The diagnostics that we use for checking our model are

- Thin-wall imaging.

- Dante.

- Burn-through strips.

Each of these diagnostics is discussed below.

Thin-Wall Imaging. We use the thin wall images described in the previous article, "Results of the Novette Implosion Experiments," to check our modeling of laser transport. What these $>6-\mathrm{keV}$ images actually measure is the relative corona emission throughout the hohlraum. It is easy to show that the local exposure of an image will be

Exposure $\sim e^{-i m / T^{*}} \times$ geometrical factors, 
Analysis of the Novette Compression Series

where $T_{\mathrm{e}}$ is the corona temperature. To back out a laser intensity from the image, we need density and temperature profiles vs laser intensity near the gold wall. LASNEX can provide us with these estimates and can also calculate how the relative exposure should vary with laser intensity. Figure 4-32 shows the relative exposure vs laser intensity that we would expect for our thin-wall images. This figure comes from a series of one-dimensional calculations and does not include geometrical factors, which should be on the order of unity for our hohlraums. Below $3 \times 10^{14}$ $W / \mathrm{cm}^{2}$, the relative exposure falls very rapidly because the corona temperature is decreasing rapidly.

Figure 4-33 shows a thin-wall image of an 0.8-scale Caim (shot No. 93110109): The cone emission is the dominant feature of the picture. Our analysis of the image (see the previous article, "Results of the Novette Implosion Experiments") indicates that the exposure everywhere else is $<1 / 30$ of the cone exposure. Thus, if the cone intensity is $\sim 1 \times 10^{15} \mathrm{~W} / \mathrm{cm}^{2}$, then the intensity in the rest of the hohlraum is $<1 \times 10^{14} \mathrm{~W} / \mathrm{cm}^{2}$. This relationship is consistent with the intensity that our model puts into the secondary (see Fig. 4-31). Note, however, that the relative exposure on the endcap is not consistent with geometrical optical transport of the laser light that passes by the scattering cones. If this light were unaffected by the cones, it would have struck the opposite endcap with an intensity of $\sim 3 \times 10^{14}$ $W / \mathrm{cm}^{2}$ in this experiment, and this intensity would have created a small, observable emission spot. This spot is not apparent in the data, so it appears that the energy that goes by the cones does not transport geometrically to the other end. If we spread the energy over the endcap, then the intensity drops to $\sim 1 \times 10^{14} \mathrm{~W} / \mathrm{cm}^{2}$, which would be consistent with the image, would be consistent with our Dante observations (described immediately below), and would not change the basic results of our modeling.

Dante. We use Dante data to check our model in two ways: to model the scaling of $x$-ray output as we change hohlraum size, laser energy, and geometry; and to see if our model properly estimates both the observed primary-secondary gradients and the

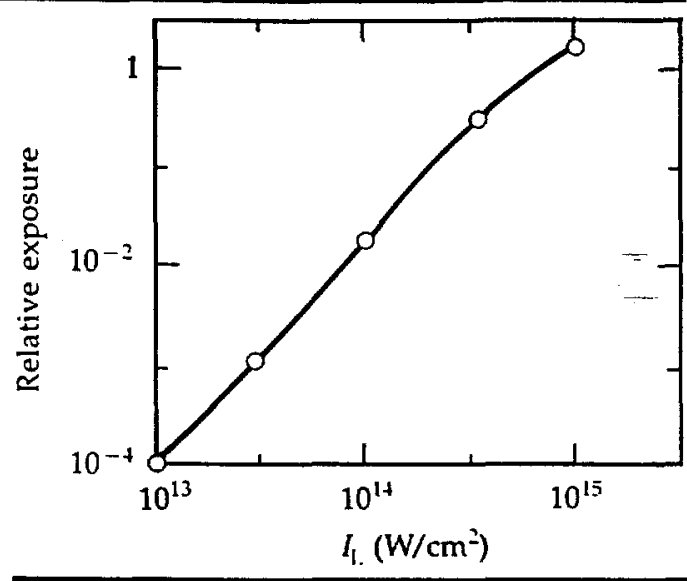

Fig. 4-32. LASNEX estimate of exposure vs laser intensity for thin-wall hohlraums.

Fig. 4-33. Thin-wall image of an 0.8 -scale Cairn.

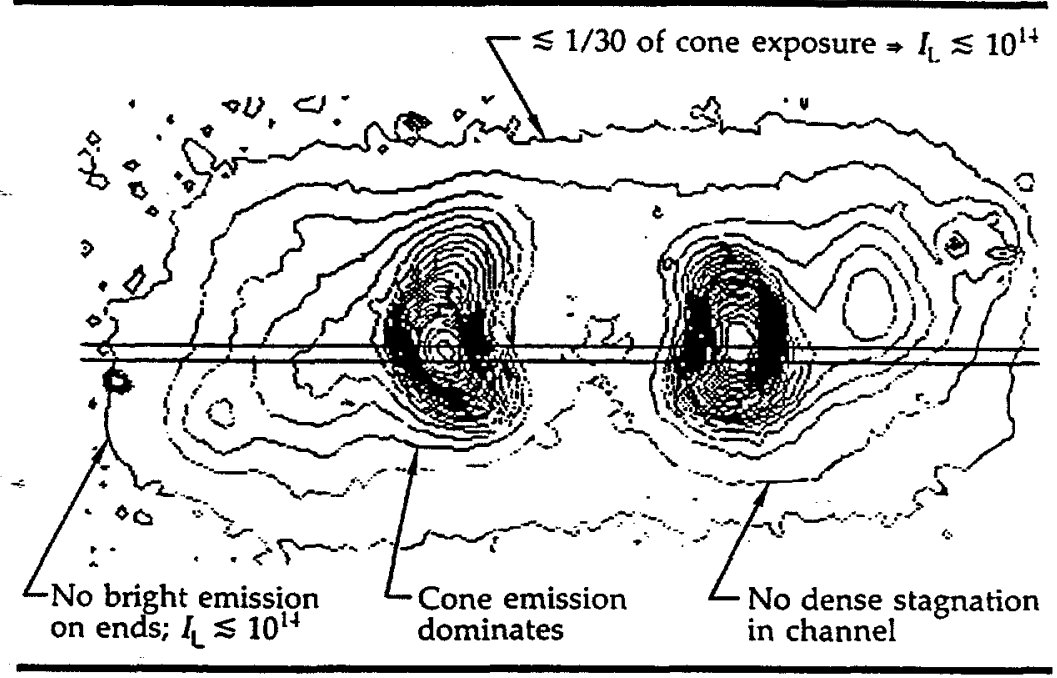

changes in these gradients. Our methods of interpreting the Dante data are described in the following article, "Analysis of Dante Data," which also gives a full discussion on our use of the channel-ratio plots and $T_{r}-$ channel plots that are mentioned below.

Our model closely predicts the change in temperature that we observed when going from a 1.0-scale full-Cairn at one energy to an 0.8-scale full-Cairn at a somewhat different energy. Figure 4-34 shows the geometry and Dante results for this particular pair of experiments (shot Nos. 93120807 and 93121905). In both these experiments, Dante $\mathrm{H}$ looked into the primary through a 400- $\mu \mathrm{m}$ hole [Fig. 4-34(a)], which was rotated down by $18^{\circ}$ so we would be less likely to be looking through hot blow-off from the cone.

Our WALLE modeling for these experiments says that Dante should have seen $130 \mathrm{eV}$ in the 1.0 -scale hohlraum and $156 \mathrm{eV}$ in the 0.8 -scale hohlraum. The actual data are close to these values. The 

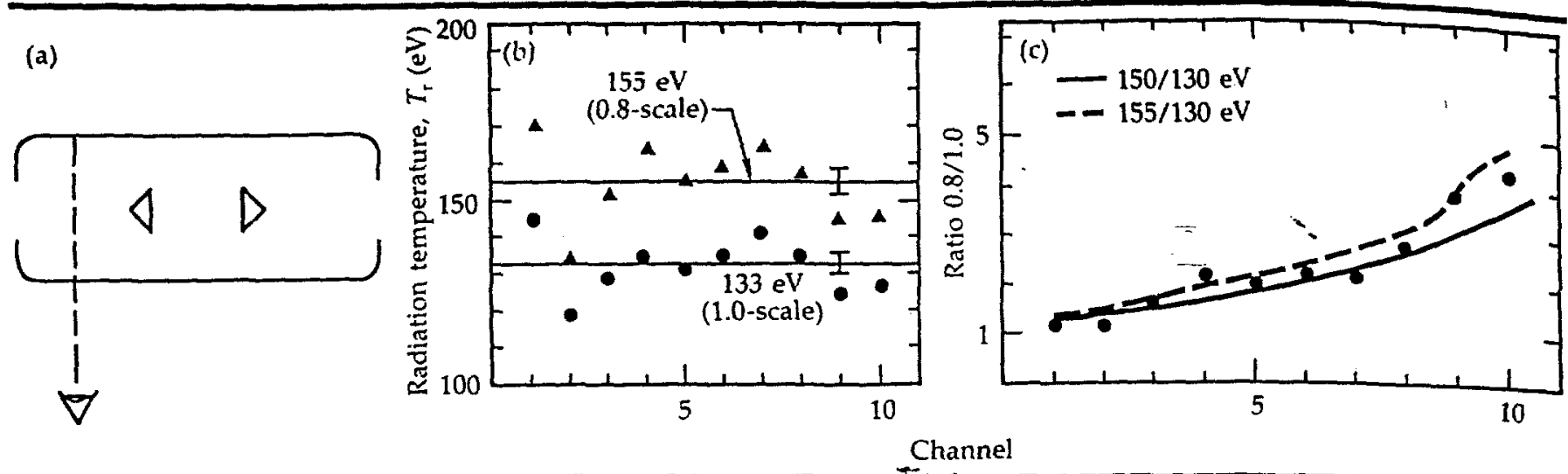

Fig. 4-34. (a) Dante $\mathrm{H}$ A line of sight into hohlraum primary.

(b) $T_{\mathrm{r}}$-channel plot of 0.8 - and 1.0-scale hohlraums.

(c) Channel-ratio plot of 0.8 - and 1.0-scale

hohlraums.

Fig. 4-35. $T_{r}$-channel plot of full- and half-Cairns.

Table 4-7. Comparison of WALLE model predictions with experimental results.

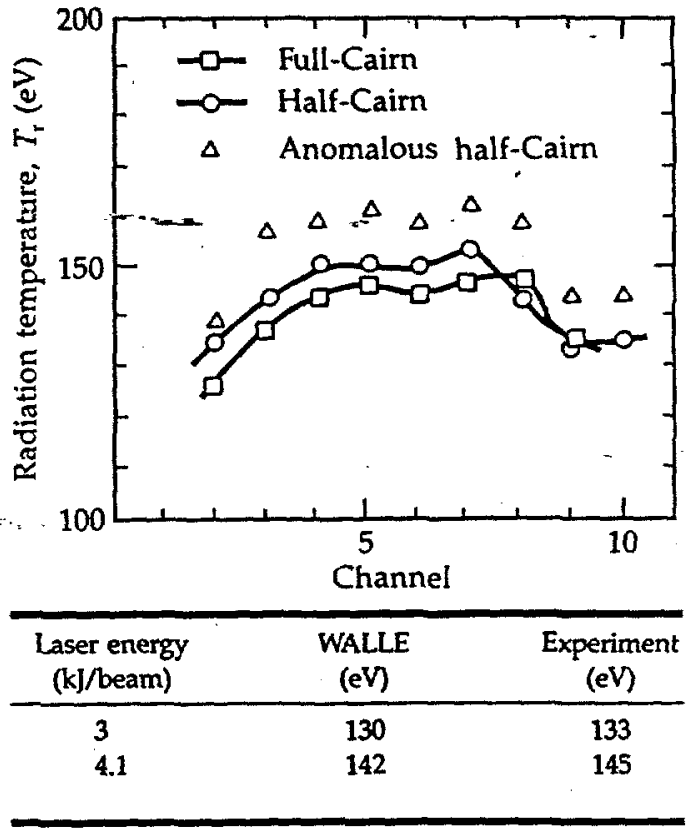

$T_{\mathrm{r}}$-channel plot shown in Fig. 4-34(b) indicates that we observed $\sim 133 \mathrm{eV}$ in the 1.0 scale hohlraum and $\sim 155 \mathrm{eV}$ in the 0.8 -scale hohlraum. The channel-ratio plot shown in Fig. 4-34(c) is consistent with the 0.8 -scale hohlraum being 20 to $25 \mathrm{eV}$ hotter than the 1.0-scale hohlraum.

If we keep the scale size fixed and vary the laser energy, our model once again closely matches the Dante observations. In another pair of experiments (shot Nos. 93121905 and 93102615), 1.0-scale hohlraums where configured as shown in Fig. 4-34, with Dante $\mathrm{H}$ looking into the primary. Table 4-7 compares our model with the experiment. The experimental values shown in the table were extracted from $T_{r^{-}}$ channel plots, and the temperature differences were checked by channel-ratio plots.

Finally, when we significantly vary the geometry, our model seems to get the scaling of Dante flux-most of the time. Figure 4-35 is a $T_{\mathrm{r}}$-channel plot for three experiments: one 1.0-scale full-Caim, and two 1.0-scale half-Cairns (shot Nos. 93102615, 93120105, and 94011009). For the full-Caim at $4.1 \mathrm{~kJ} /$ beam, WALLE predicted $142 \mathrm{eV}$, and the experiment, with Dante $\mathrm{H}$ looking into the primary, gave $145 \mathrm{eV}$. Now if we change geometry to a half-Cairn and fire $3.8 \mathrm{~kJ}$ of energy into it, our model says that Dante $H$ should see $\sim 145 \mathrm{eV}$. The observation on one of the half-Cairns was $148 \mathrm{eV}$, a few volts hotter. This sort of result has generally been true of the half-Caim experiments that we modeled: WALLE closely predicts the temperature. However, the second half-Caim experiment (shot No.

94011009) is anomalous; Dante saw a temperature that was $10 \mathrm{eV}$ hotter than can be explained by our model-or by simple scaling or by any other model. The $T_{\mathrm{r}}$-channel data for this anomalous experiment are also shown in Fig. 4-35.

Dante data were also used to check our modeling of primary-secondary gradients in both 1.0- and 0.8-scale hohlraums. We will discuss the gradients in 1.0-scale hohlraums first.

The first experiment that we compared with our modeling was a 1.0-scale hohlraum (shot No. 93102615), which probably was the cleanest gradient experiment in the series. The geometry of this experiment is shown in Fig. 4-36(a). We claim that this was the cleanest experiment because Dante $\mathrm{H}$ looked into the primary, while Dante A looked into the secondary. Thus, the experiment has none of the problems (discussed below) that exist when Dante A looks into the primary. In all the other experiments described below, the Dantes 
Analysis of the Novette Compression Series
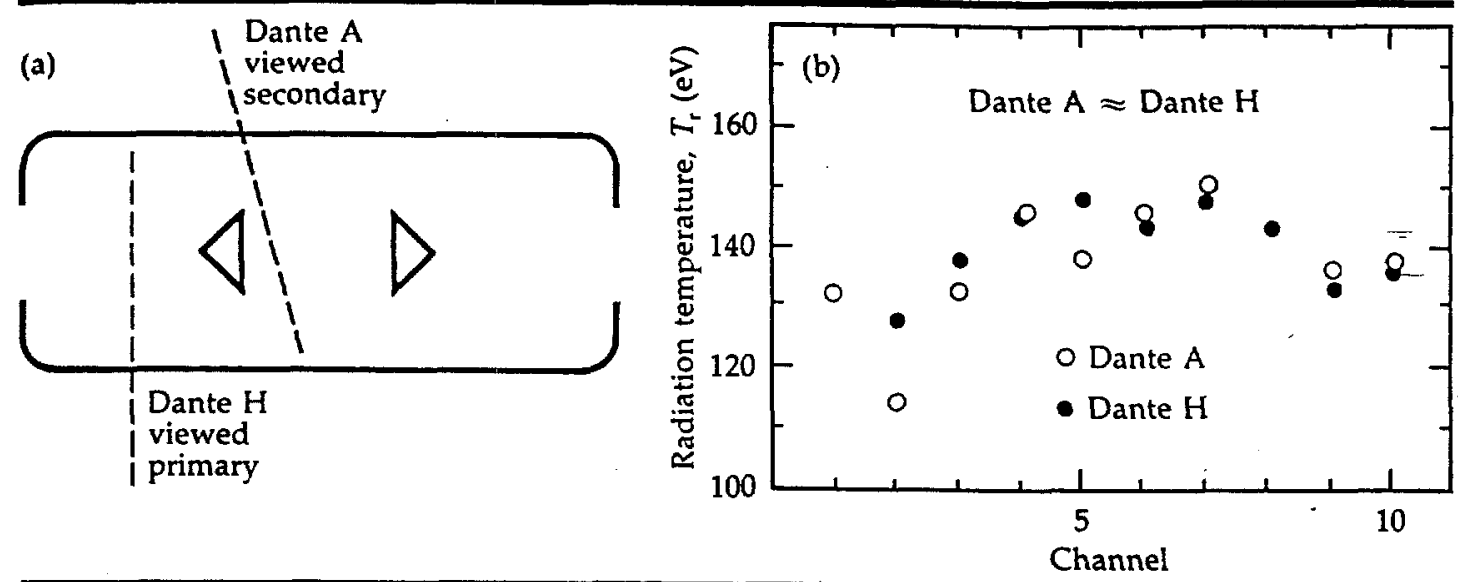

were reversed from this experiment, so that Dante $A$ looked into the primary and Dante $\mathrm{H}$ looked into the secondary.

With the geometry shown in Fig. 4-36(a), and with $4.1 \mathrm{~kJ}$ per beam, our model predicts that Dante A should see $139 \mathrm{eV}$ in the secondary and Dante $\mathrm{H}$ should see $142 \mathrm{eV}$ in the primary. The experiment is close to this prediction, with both Dante A and Dante $\mathrm{H}$ reading $\sim 140 \mathrm{eV}$. The $T_{\mathrm{r}}-$ channel plot for the two Dantes is show in Fig. 4-36(b).

The geometry of the second 1.0 -scalehohlraum gradient experiment (shot No. 93101313) is shown in Fig. 4-37. Our nominal WALLE modeling predicts that, with $4.5 \mathrm{~kJ} /$ beam, the secondary should be slightly cooler than the primary (143 vs $145 \mathrm{eV}$, respectively). The experiment, however, provides a different result: the data indicate that the secondary is hotter than the primary, not cooler.

One way to explain this apparent hotter temperature in the secondary is to say that, on this experiment, even more laser light than we normally assume was scattered into the secondary. However, we can also explain the hotter temperature by invoking beam misalignment, a fairly likely possibility with Novette. Figure 4-38 shows a 1.0-scale hohlraum with the beam opposite the Dante A side severely misaligned.

When we model this situation, we find that misalignment can significantly affect what is seen by Dante A. As the figure indicates, if tbe beam is misaligned so it hits on the side opposite that being viewed by Dante $A$, then the primary might indicate $138 \mathrm{eV}$ while the secondary would indicate $143 \mathrm{eV}$. Thus, beam misalignment could make the
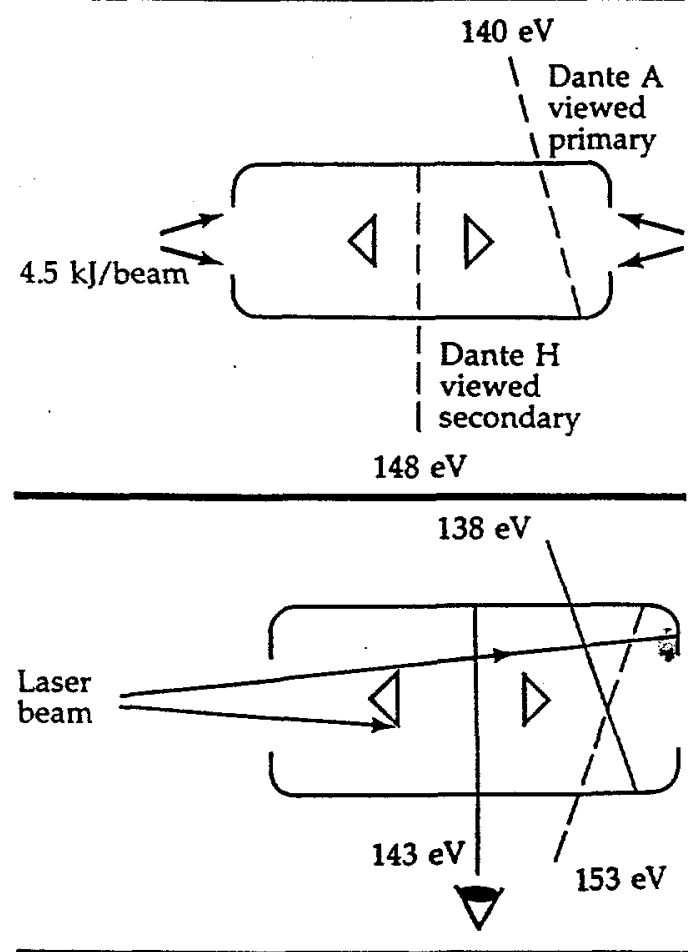

primary appear cooler than the secondary,

which is consistent with the experiment.

Figure 4-38 also points out that, when we view the primary with Dante $A$, great variations in the measurement are possible. If the beam were misaligned in the opposite sense, so that most of the laser light struck on the side seen by Dante $A$, then we might have measured $\sim 153 \mathrm{eV}$ in the primary. Our model says that beam misalignment can make the primary appear to be anywhere between $\sim 138$ and $153 \mathrm{eV}$ when viewed with Dante A. By contrast, viewing the same experiment with Dante $H$ (as we did in the experiment shown in Fig. 4-36) would result in far less sensitivity to misalignment; under the same conditions,
Fig. 4-36. Geometry of first Dante experiment on 1.0-scale hohlraum. (b) $T_{\mathrm{r}}$-channel plot for both Dantes.

Fig. 4-37. Geometry of second Dante experiment on 1.0 -scale hohlraum.

Fig. 4-38. Our model indicates that Dante $A$ could see primary temperatures anywhere between 138 and $153 \mathrm{eV}$, depending on how the beam is misaligned. 
Dante $\mathrm{H}$ is predicted to have ranged between $\sim 144$ and $146 \mathrm{eV}$.

When we go to 0.8-scale hohlraums, it turns out that there is another problem with Dante A primary temperatures. In the 0.8 scale hohlraums, Dante $A$ also sees a piece of gold that, under geometric optics, would be directly heated by the laser (see Fig. 4-39), thus making the primary appear hot.

To check primary-secondary gradients in 0.8-scale hohlraums, we shot two 0.8 scale thin-wall empty hohlraums (shot Nos. 93091503 and 93110109) and made primarysecondary Dante measurements. Thesexperiments were shot with different focusings. In the first experiment, we overfilled the $480-\mu \mathrm{m}$ cone with a $680-\mu \mathrm{m}$ spot, as shown in Fig. 4-39(a). In the second experiment, we tried to underfill the cone with a $400-\mu \mathrm{m}$ spot, but it turns out that the beam was misaligned [Fig. 4-40(a)], as observed by the pinhole cameras and the microchannel plate-pinhole camera. In both experiments, energy that missed the scattering cone became an important part of interpreting the data.

In the first experiment, where we overfilled the cone (shot No. 93091503), the primary appears $\sim 15$ to $20 \mathrm{eV}$ hotter than the secondary. Dante $\mathrm{H}$ indicates that the secondary was $\sim 146$ to $149 \mathrm{eV}$, with the range coming from different ways of converting the Dante data to a temperature (see the following article, "Analysis of Dante Data"). Dante A indicated that the primary was $\sim 161$ to $170 \mathrm{eV}$. Figure 4-39(b) shows the channel-ratio plot for this experiment, which corroborates a $15-$ to $20-\mathrm{eV}$ temperature gradient.

At first glance, our WALLE modeling for this experiment appears to produce a somewhat smaller temperature gradient. We initially estimated a 7-eV gradient, with the primary coming to $\sim 161 \mathrm{eV}$ and the secondary to $\sim 154 \mathrm{eV}$. However, our modeling does not take into account the increase in flux that we would expect Dante $A$ to get from the directly irradiated piece of endcap. Thus, our prediction of $161 \mathrm{eV}$ probably should be considered a lower bound on the primary temperature. For this 0.8-scale hohlraum, then, our model predicts that Dante A should see $>161 \mathrm{eV}$ in the primary and Dante $H$ should see $\sim 154 \mathrm{eV}$ in the secondary. These values are in reasonably good agreement with the experiment ( $\sim 161$ to $170 \mathrm{eV}$ in the primary, and $\sim 146$ to $149 \mathrm{eV}$ in the secondary).

On the second experiment with an 0.8 scale hohlraum (shot No. 93110109), we used tight focusing, but the beam was misaligned (Fig. 4-40). The key observational
Fig. 4-39. (a) First 0.8scale hohlraum, with Dante A viewing some of the directly irradiated gold in the overfilled hohlraum. (b) Channel-ratio plot for this experiment.

Fig. 4-40. (a) Second 0.8-scale hohlraum, where the cones were underfilled and the west beam was observed to be misaligned, as shown. (b) $T$-channel plots for this experiment.
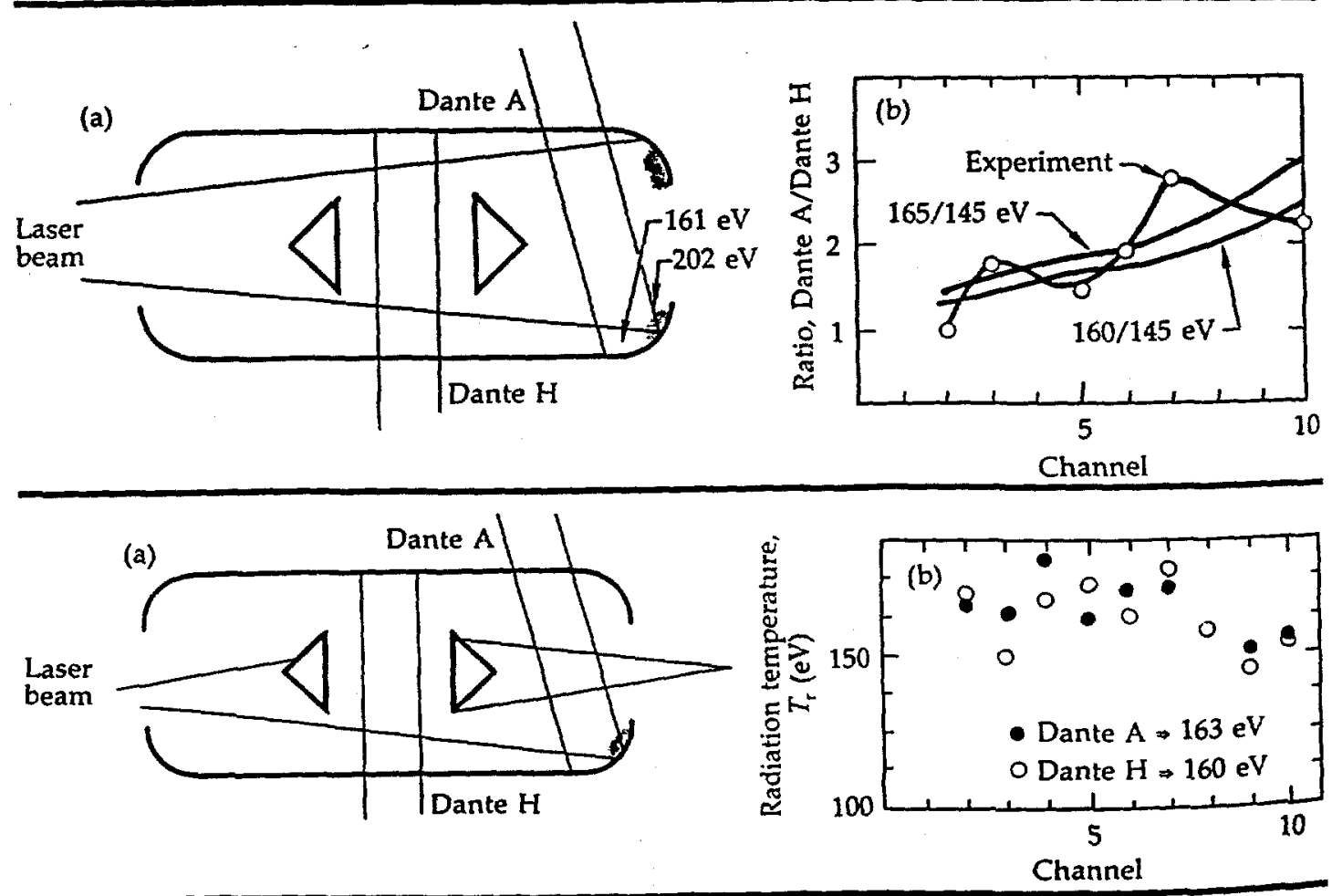
Analysis of the Novette Compression Series

change in this experiment is that the secondary was no longer significantly cooler than the primary. Figure 4-40(b) shows the $T_{r}$-channel plot for this experiment; the data look too close to call. The temperatures we quote for the shot are $163 \mathrm{eV}$ for the primary and $160 \mathrm{eV}$ for the secondary. Our WALLE modeling for this hohlraum, where we take into account the beam misalignment that was independently observed by the microchannel plate-pinhole camera, estimates $>154 \mathrm{eV}$ for the primary and $162 \mathrm{eV}$ for the secondary. These estimates are also reasonably consistent with the experiment.

The fact that our model properly estimates the change in primary-secondary gradient when we change focusing is an important result. In the model, the change comes about because a greater fraction of the beam energy strikes the cone when we decrease the spot size, increasing the amount of energy scattered into the secondary (see Fig. 4-29) and raising the temperature seen by the Dante viewing that area. The ability to estimate the change in gradients when we change spot size corroborates our transport model, which is important because the direct result of this transport model is capsule-drive asymmetry.

In summary, our modeling is generally consistent with the Dante data. We closely model the changes in Dante fluxes as we change hohlraum scale, laser energy, and geometry. Our model plausibly explains the primary-secondary gradients that we observe, especially if we are free to invoke the beam misalignment that we know occurs. The Dante observations appear consistent with light being scattered into

the secondary.

Burn-Through Strips. A burn-through

strip is a $1-\mu \mathrm{m}$-thick strip of gold that was built into our hohlraums to provide information about energy-deposition gradients along the wall (see the previous article in this section, "Results of the Novette Implosion Experiments," and the article "Bum-Through Foil and Witness-Plate Experiments for Novette" in Section 2). Figure 4-41 shows the estimate given by our model of incident power vs time at several locations along the burn-through strip; each of these locations receives a different mixture of laser power and radiation. We compare our model with data for the burnthrough strip by estimating a burn-through time for each of the radiation/laser combinations that we think we have along the wall. This estimate is done with LASNEX simulations (see the article "Burn-Through Foil and Witness-Plate Experiments for Novette" in Section 2).

Figure 4-42 compares measured burnthrough times (shot Nos. 93100303, 93100405, and 93100504) with the predictions of our model. The data, taken from the previous article, "Results of the Novette Implosion Experiments," are the relative burn-through time vs position along the strip. (By relative burn-through time, we mean the time difference between burnthrough at a given point and the burnthrough that was first observed anywhere on the strip.) Figure 4-42 also shows the predictions of our model. We have plotted two different model curves on the figure

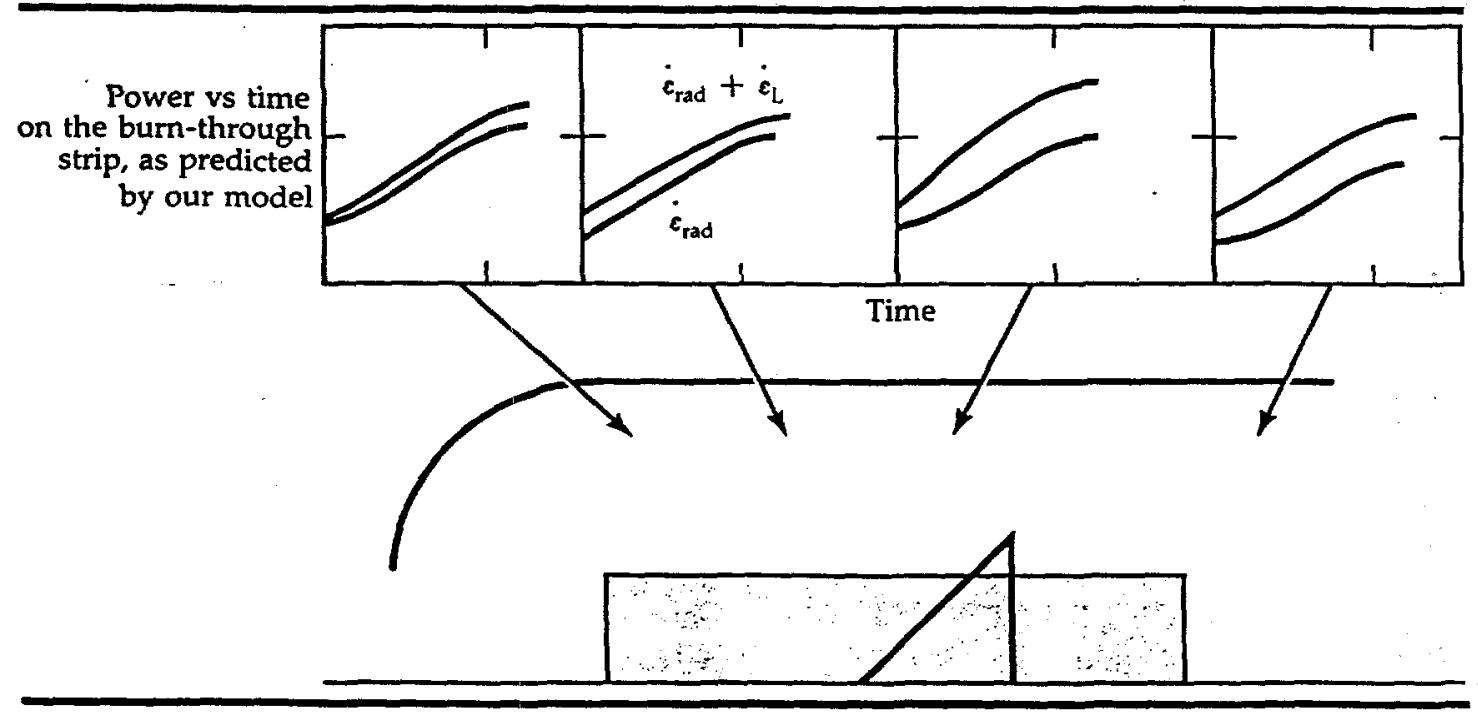

Fig. 4-41. Power vs time deposited on the inside of the burnthrough strip, as predicted by our model. 
Analysis of the Novette Compression Series

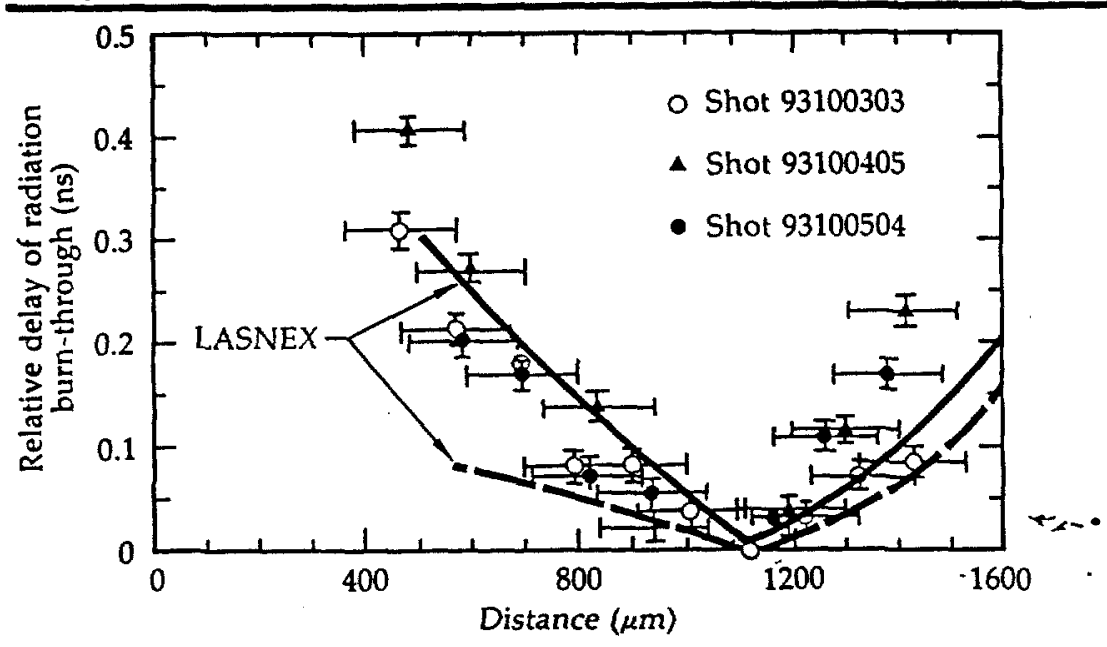

predictions of capsule flux out of the hohlraum wall (see Fig. 4-31). WALLE allows us to properly integrate this flux to obtain the capsule drive and asymmetry. The results of this analysis are given in Table 4-8, in which the drive values represent the average peak flux onto the capsule as calculated by our WALLE model. We must emphasize that our modeling is likely to be a lower bound on the flux, since our model is normalized to scaling concepts that have systematically neglected hole closure.

The plus value for the flux asymmetry in Table 4-8 means that the capsule waist receives more flux than the poles; this asymmetry would produce a sausage-like implosion. The asymumetry results from laser energy getting into the secondary, making the wall of the hohlraum above the capsule hotter than the backs of the cones (see Fig. 4-31) and producing a net $P_{2}$ asymmetry.

During assembly of the hohlraums, a certain amount of glue ends up on the back of the scattering cones. The last column in Table 4-8 is the estimate by our model of how this glue worsens the flux asymmetry on the capsule. The effects of the glue are worse in the 0.8-scale hohlraums than in the 1.0-scale hohlraums. We discuss the glue in more detail later.

Finally, the row labeled Stretch 0.8 in Table 4-8 represents a hohlraum that we never shot. According to our model, we would have good drive and little asymmetry (under our previously described assumptions of axisymmetry) if we stretched the secondary length to $925 \mu \mathrm{m}$ and kept the capsule from seeing the glue on the back of the cones.

Discussion of Drive and Asymmetry. Besides our semiempirical hohlraum model, we have also tried a variety of other hohlraum models. The basic result is that all scenarios that put laser energy into the secondary, but not onto the backs of the. cones, produce a sausage-like drive asymmetry. For example, if we assume that all the light that misses, or is scattered by, the cones is distributed uniformly along the primary and secondary walls, then we end up with a flux asymmetry of $\sim 8 \%$. If we tend to concentrate the nonabsorbed light in the secondary, then the asymmetry rises even further.

The drive we estimate for the 0.8-scale

When we try to understand capsule implosions, the most important part of our comiammirieal hnhlraim model is ite 
Analysis of the Novette Compression Series

the value of $172 \pm 5 \mathrm{eV}$ that we infer from a witness plate on one of the subscale hohlraums. However, we can reconcile the two values with a few considerations. The witness plate on the 0.8-scale hohlraum was shot with a $600-\mu \mathrm{m}$ spot, not our nominal $680-\mu \mathrm{m}$ spot. Our model says that this smaller spot would raise the average drive temperature by $\sim 4 \mathrm{eV}$. Another $2-\mathrm{eV}$ correction is made for the witness-plate orientation, which corresponds to the part of the capsule that receives the most flux. Our model, then, says that the witness plate should have seen $\sim 156+4+2 \approx 162 \mathrm{eV}$. If we add in another $\sim 8 \mathrm{eV}$ to correct for hole closure and for the Dante temporal response (see the following article, "Analysis of Dante Data"), then our model and the witness plate are reconciled.

Capsule Yield. Figure 4-43 shows a LASNEX plot of neutron yield vs $T_{r}$ for capsules having nominal dimensions of $200 \mu \mathrm{m}$ in diameter by $5 \mu \mathrm{m}$ thick, with a 24- $\mu \mathrm{m} \mathrm{CH}$ coating and a gas fill of $10 \mathrm{mg} / \mathrm{cm}^{3}$. On this plot, we have indicated the experimental yields for implosions done in 1.0- and 0.8-scale hohlraums, as well as our estimates of capsule drive. The yields in our 1.0-scale hohlraums are down by a factor of 2 to 10 from our predictions. (The range in our predicted yields arises from our suspicion that the WALLE drives are a lower bound on radiation temperature due to our neglect of hole-closure effects on Dante data.) However, in the 0.8-scale hohlraums, the experimental yields are down by more than 100 from our LASNEX yields. We have serious problems in the 0.8-scale hohlraums.

Asymmetry Scenario. Our guess is that flux asymmetry, or possibly mix exacerbated by asymmetry, is the most probable cause for the reduced yields in the 0.8-scale hohlraums. To make a convincing case that asymmetry is the cause, we will show that: - There probably was more asymmetry in the 0.8 -scale hohlraums than in the 1.0scale hohlraums. This difference is necessary if asymmetry is to explain why yields of the 0.8-scale hohlraums were so much farther below LASNEX predictions than those of the 1.0-scale hohlraums.

- The capsules are sensitive to asymmetry.

- Our high areal density ( $p r)$ can be consistent with an asymmetry scenario.

Our nominal WALLE modeling says that the inherent asymmetry in the 0.8 -scale

\begin{tabular}{ccccc}
$\begin{array}{c}\text { Hohlraum } \\
\text { scale }\end{array}$ & $\begin{array}{c}\text { Drive } \\
(\mathrm{eV})\end{array}$ & $\begin{array}{c}\text { Asymmetry } \\
(\%)\end{array}$ & $\begin{array}{c}\text { Asymmetry } \\
\text { with glue } \\
(\%)\end{array}$ & $\begin{array}{l}\text { Table 4-8. WALLE } \\
\text { predictions of capsule } \\
\text { drive and flux } \\
\text { asymmetry. }\end{array}$ \\
\cline { 1 - 4 } 1.0 & $>138$ & +8 & $>10$ & \\
0.8 & $>156$ & +8 & $>15$ & \\
Stretch 0.8 & $>153$ & $<1$ & $<1$ & \\
\hline
\end{tabular}

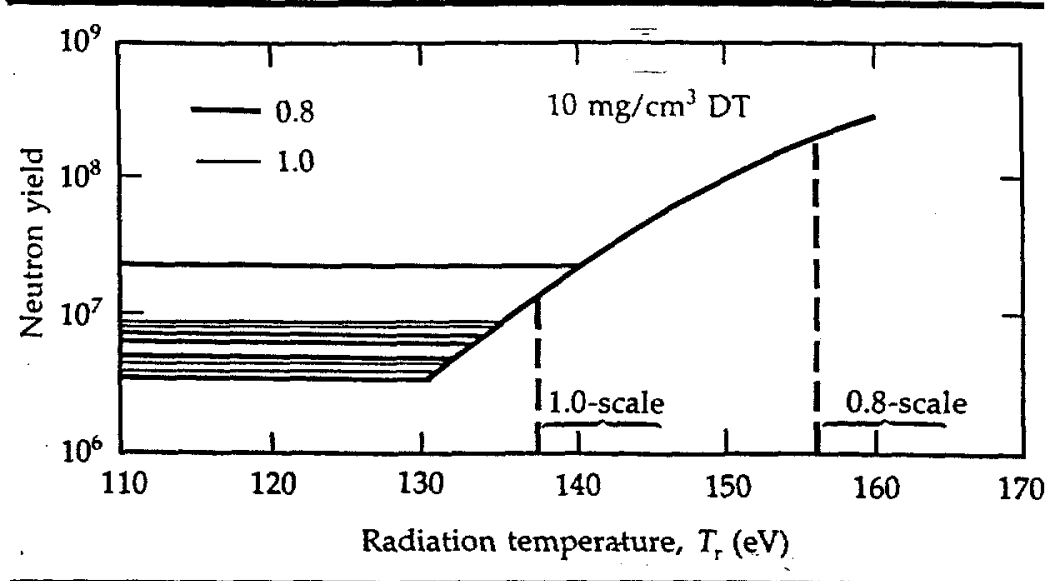

hohlraums was the same as in the 1.0-scale hohlraums; both produce about $+8 \%$ asymmetry. However, there is a glue perturbation, not included in our model, that would make the asymmetry worse in the 0.8-scale hohlraums.

Figure 4-44 shows how the scattering cones were glued to two $15-\mu \mathrm{m}$ tungsten wires when the cones were mounted in the hohlraums. The assembly technique was the same for both scale sizes. ${ }^{35}$. A drop of glue was placed on each wire, the cone was brought up to the wires, and, usually, the cone had to be moved around to center it. Some assembly photographs of 1.0-scale hohlraums indicate that 20 to $25 \%$ of the cone backs were covered with a glue layer of unknown thickness. There are no comparable photographs of 0.8-scale hohlraums, but, since the mass of glue was the same for these as it was for the 1.0-scale hohlraums (one drop per wire), it is reasonable to assume that the glue comprised a larger or thicker perturbation on the 0.8 scale cones. (The cone-back area of an 0.8 scale hohlraum is 0.64 times that of a 1.0-scale hohlraum.)

LASNEX estimates of the interaction of glue with radiation indicate that, on a 1-ns time scale, glue has an albedo of $\sim 0.2$ relative to gold and that the radiation will burn through $\sim 20 \mu \mathrm{m}$ of glue during the experiment. Table 4-9 shows how various amounts of thick $(>20 \mu \mathrm{m})$ glue affect the flux asymmetry on a hohlraum of either scale. Our semieducated guess is that flux asymmetry was probably between 10 and
Fig. 4-43. LASNEX. predictions of neutron yield vs $T_{\mathrm{r}}$ for our capsules. Horizontal lines indicate experimental yields; brackets on abscissa show apparent drive temperatures for 0.8 - and 1.0-scale hohlraums. 
Fig. 4-44. Scattering cones glued to their support wires.

Table 4-9. Effect of glue on flux asymmetry.

Analysis of the Novette Compression Series
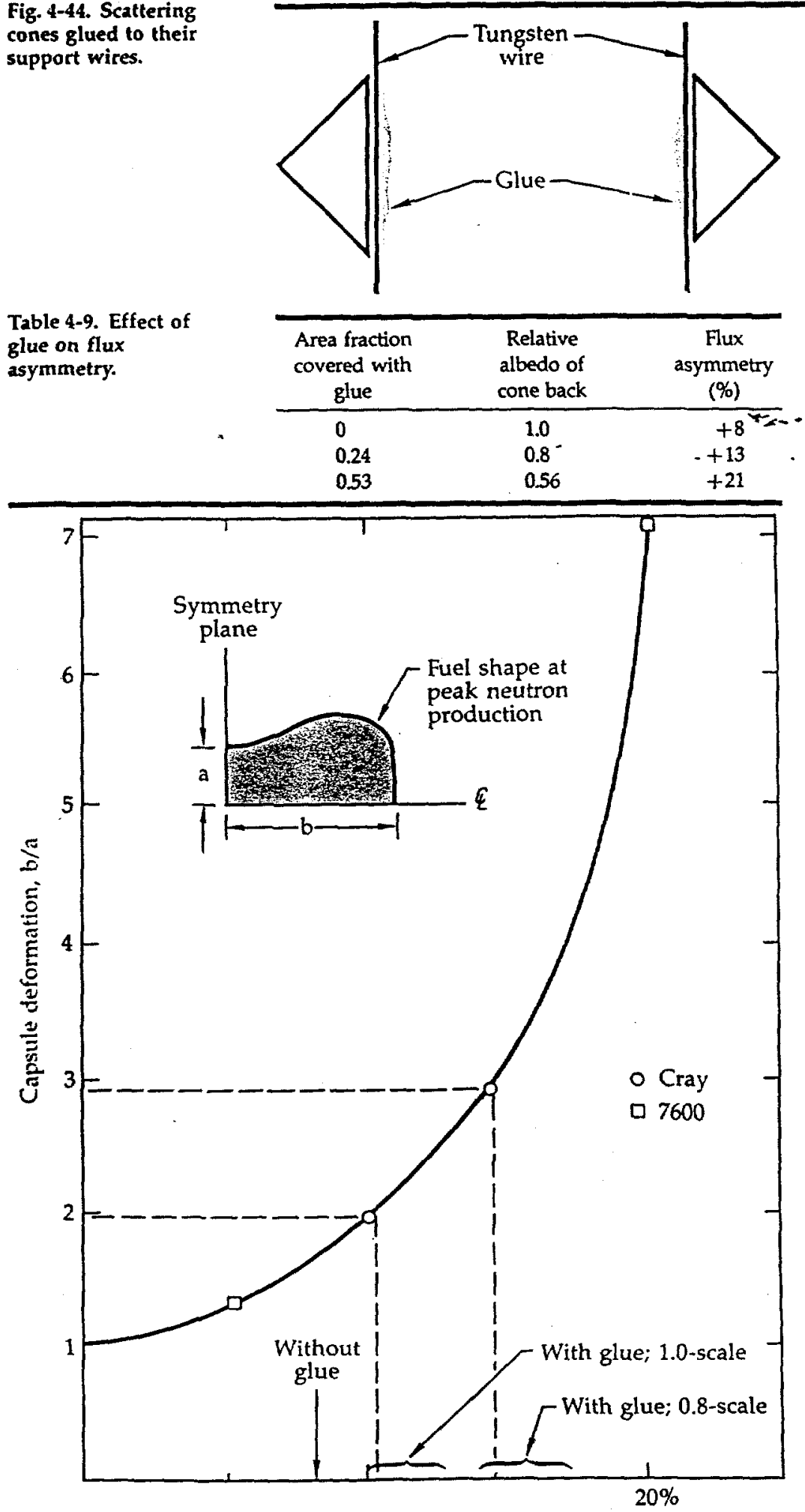

Flux asymmetry

Fig. 4-45. LASNEX estimate of capsule deformation vs asymmetry. Deformation. $b / a$, is defined in the small inset figure.
$13 \%$ on the 1.0 -scale hohlraums and around 15 to $18 \%$ on the 0.8 -scale hohlraums.

Figure $4-45$ is a LASNEX-generated plot of capsule deformation (defined in the smaller illustration) vs drive asymmetry. This figure resulted from a series of twodimensional simulations done on both the
Cray and the 7600 of $200-\mu \mathrm{m}$-diam $\times 6.1$ $\mu \mathrm{m}$-thick capsules coated with $21.7 \mu \mathrm{m}$ of $\mathrm{CH}$ and filled with $10 \mathrm{mg} / \mathrm{cm}^{3}$ of DT gas. According to these simulations, the capsule implosions are in the sensitive-toasymmetry regime when flux asymmetries are greater than about' $10 \%$.

Our attempts to explain yield degradation by asymmetry are based on the curve shown in Fig. 4-45. The 1.0-scale capsules would have been deformed $\sim 2: 1$ if they had $\sim 10 \%$ flux asymmetry. If the asymmetry went up to $\sim 15 \%$ in the 0.8 -scale capsules, then these implosions would have been deformed $\sim 3: 1$. This significantly greater deformation of the 0.8 -scale hohlraums is a possible reason why they performed so poorly relative to our expectátions:

LASNEX does not show much yield sensitivity due to flux asymmetry. Even at an asymmetry of $20 \%$, the part of the calculated yield reduction that can be attributed to asymmetry is closer to a factor of 2 than it is to 100 . This calculational insensitivity to asymmetry, however, does not agree with experiment.

In our test series, we inadvertently performed some shots that gave results indicating that the yield is surprisingly sensitive to asymmetry. These experiments (shot Nos. 93100504, 93111635, and 93120704) are summarized in Fig. 4-46. In the first experiment, we clipped off about half of one beam on the laser entrance hole of a 1.0-scale hohlraum. The yield on this shot was $\sim 2 \times 10^{5}$ neutrons, down by a factor of 30 from the average of $\sim 6 \times 10^{6}$ neutrons obtained for 1.0-scale hohlraums. The other two shots were 0.8 -scale hohlraums where one of the two beams went at about half energy. The yields in both of these shots were down by a factor of $\sim 60$ from the nominal yields for these smaller hohlraums.

Figure 4-46 also shows the flux asymmetry that we estimate for an 0.8 -scale hohlraum when the energy in one beam is half that of the other beam. This asymmetry is small, with the $P_{1}$ and $P_{2}$ components both being $\sim 10 \%$. The conclusion we draw from this estimate, coupled with the low yields, is that the capsules show a gross yield sensitivity to asymmetry that is far more severe than we would expect from LASNEX asymmetry calculations.

The fractional increases in capsule $\rho r$ values that we measured in the Novette series 
Analysis of the Novette Compression Series

were significantly higher than we had previously observed on Shiva and Argus (see the previous article, "Results of the Novette Implosion Experiments"). These higher or values, presumably, are due mostly to the reduction in hot-electron preheat that we achieved by decreasing the laser wavelength, but these $\rho r$ values can also be consistent with the asymmetry scenario that we have been developing in this article. We show this consistency by invoking asymmetry as a rationale for cutting off our implosions at less than full one-dimensional convergence. Because of the greater asymmetry in 0.8-scale hohlraums, we will allow ourselves to cut off implosions in these hohlraums at a lower convergence.

Figure 4-47 plots fractional change in pusher areal density vs yield for implosions of our gas-filled capsules. We have also plotted in this figure some lines extracted from our one-dimensional calculations; these lines show the trajectory in pr ratio vs yield space that is swept out by a capsule as it implodes and produces yield. The numbers highlighted next to each line show the pusher convergence that the calculation had achieved at each point on the line. For example, the first point on the line labeled $160 \mathrm{eV}$ means that the neutron-averaged $\rho r$ ratio was $\sim 30$ and the yield was $\sim 4 \times 10^{6}$ neutrons when our $160-\mathrm{eV}$ calculation had 9-fold convergence. Slightly later in the implosion, the convergence rose to 9.5 , and the $\rho r$ ratio and yield went up to 40 and $2 \times 10^{7}$ neutrons, respectively.
Our asymmetry scenario, then, is that the 1.0-scale hohlraums are imploded at greater than $\sim 135 \mathrm{eV}$ and achieve an equivalent convergence of 10 to 12 . At that point, the experiment is quenched by asymmetry (i.e., the target prematurely stops producing yield for a reason that we do not
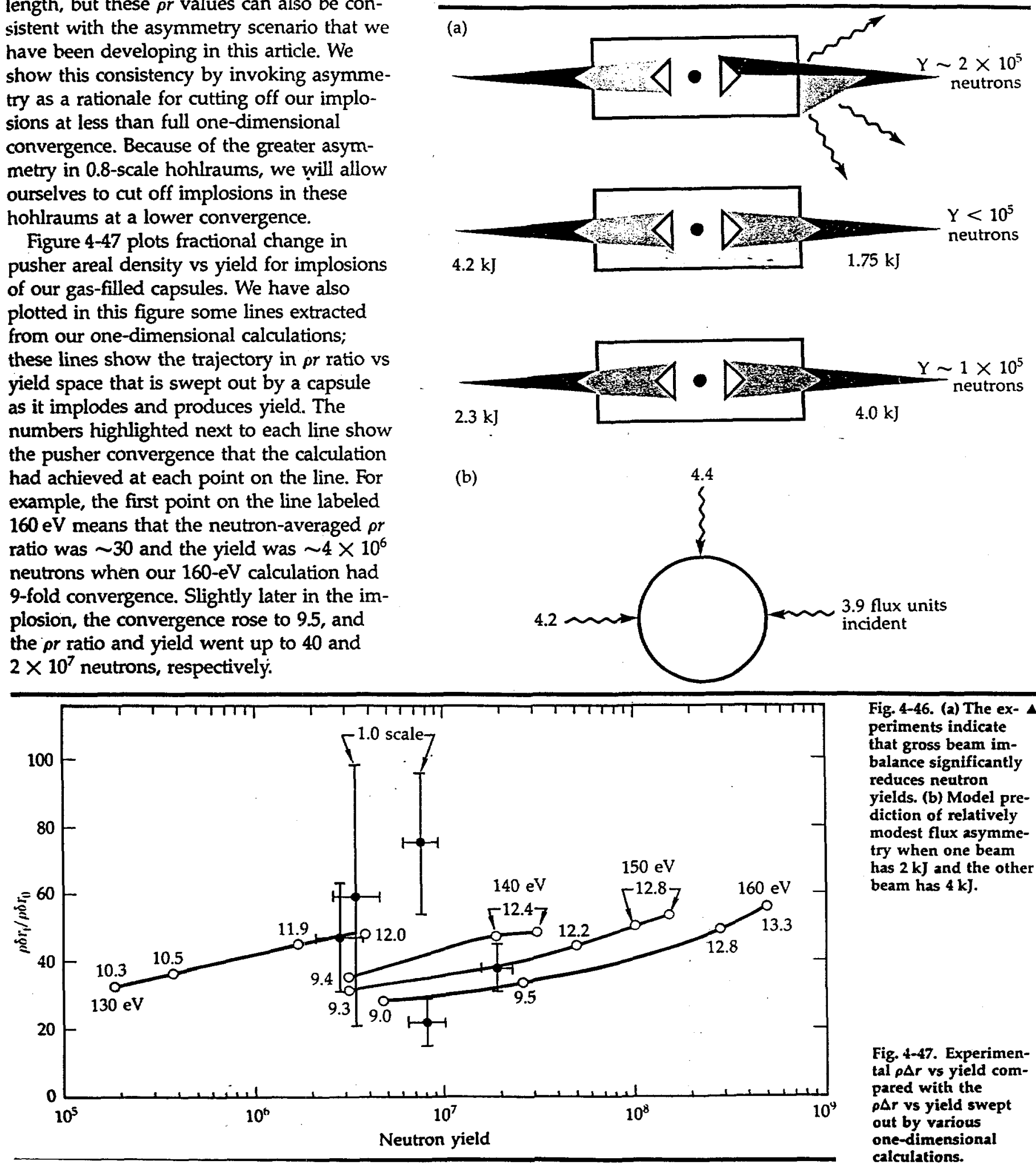

Fig. 4-46. (a) The ex- 4 periments indicate that gross beam imbalance significantly reduces neutron yields. (b) Model prediction of relatively modest flux asymmetry when one beam has $\mathbf{2} \mathbf{~ J J}$ and the other beam has $4 \mathrm{~kJ}$. 
Analysis of the Novette Compression Series

understand, but that we think is caused by asymmetry). The 0.8 -scale hohlraums are imploded at greater than $\sim 155 \mathrm{eV}$ and are quenched at an equivalent convergence of $\sim 9$ to 10 . If this scenario is what actually happened, then our implosions achieved densities of 10 to $17 \mathrm{~g} / \mathrm{cm}^{3}$ in the 1.0 -scale hohlraums and 7 to $10 \mathrm{~g} / \mathrm{cm}^{3}$ in the 0.8 -scale hohlraums.

The two leading causes for quenching by asymmetry are

- The effects of the distortion, and possibly .. even the distortion itself, are far greater than LASNEX predicts.

- Asymmetry induces mix, which reduces the DT temperature at a given convergence.

Although we believe the asymmetry scenario offers the most likely explanation forlow yields in the 0.8-scale hohlraums, there is another possible scenario, the mix-only scenario, that we explore below.

Mix-Only Scenario. We are reluctant to directly invoke mix as a cause for reduced yield for two reasons. First, we did not need to invoke it on NPIRE $1 .{ }^{36}$ Second, our rudimentary efforts to model mix have produced results that disagree with the experiments. In the NPIRE I series, we shot capsules that seem to be essentially identical to our Novette capsules, yet the NPIRE I capsules produced yields close to those predicted by one-dimensional analyses. Also, the NPIRE I drive was $\sim 140 \mathrm{eV}$, which is close to our present 1.0-scale hohlraum drives. Subsequent analysis of NPIRE I, made with our current version of WALLE, indicates that the flux asymmetry in that series is likely to have been $<6 \%$. Considering the NPIRE I analysis together with our current analysis of the Novette series makes us argue that our capsules, shot at $\sim 140 \mathrm{eV}$, may not always be subject to mix degradation. The fact that our modeling indicates better symmetry for the NPIRE I experiments clearly suggests that flux symmetry is the cause for the reduced yields on Novette.

Figure 4-48 shows that our Novette results are inconsistent with simple mix modeling. The figure plots $\rho r$ vs yield for our 1.0- and 0.8-scale experiments, as well as one-dimensional LASNEX estimates of how $\rho r$ and yield vary as we mix glass into the fuel. The ratios on the lines indicate the number of DT. fuel masses of glass that we uniformily mixed into the fuel at 140 and $160-\mathrm{eV}$ capsule drives. In these calculations, we assumed that the glass was uniformly mixed into the fuel from the start of the implosion.

At $140-\mathrm{eV}$ drive, and nominally mocking. up the 1.0-scale hohlraums, we can get good agreement with the data by invoking about one fuel mass of mix. This amount of mix lowers the yield and raises the $\rho r$ slightly into just the right regime. When we next try to simulate the 0.8 -scale hohlraums by increasing the drive to $160 \mathrm{eV}$, we find that we can match the yields with about three fuel masses of mix. However, this mix also raises the $\rho r$ values, making them greater than those for the 1.0-scale hohlraums, an obvious disagreement with the data.

More sophisticated efforts ${ }^{37}$ to dynamically model mix have led to the same results as above. A one-dimensional pure mix
Fig. 4-48. Experimental $\rho r$ vs yield and LASNEX-predicted $\rho r$ vs yield for various amounts of glass mixed into fuel.

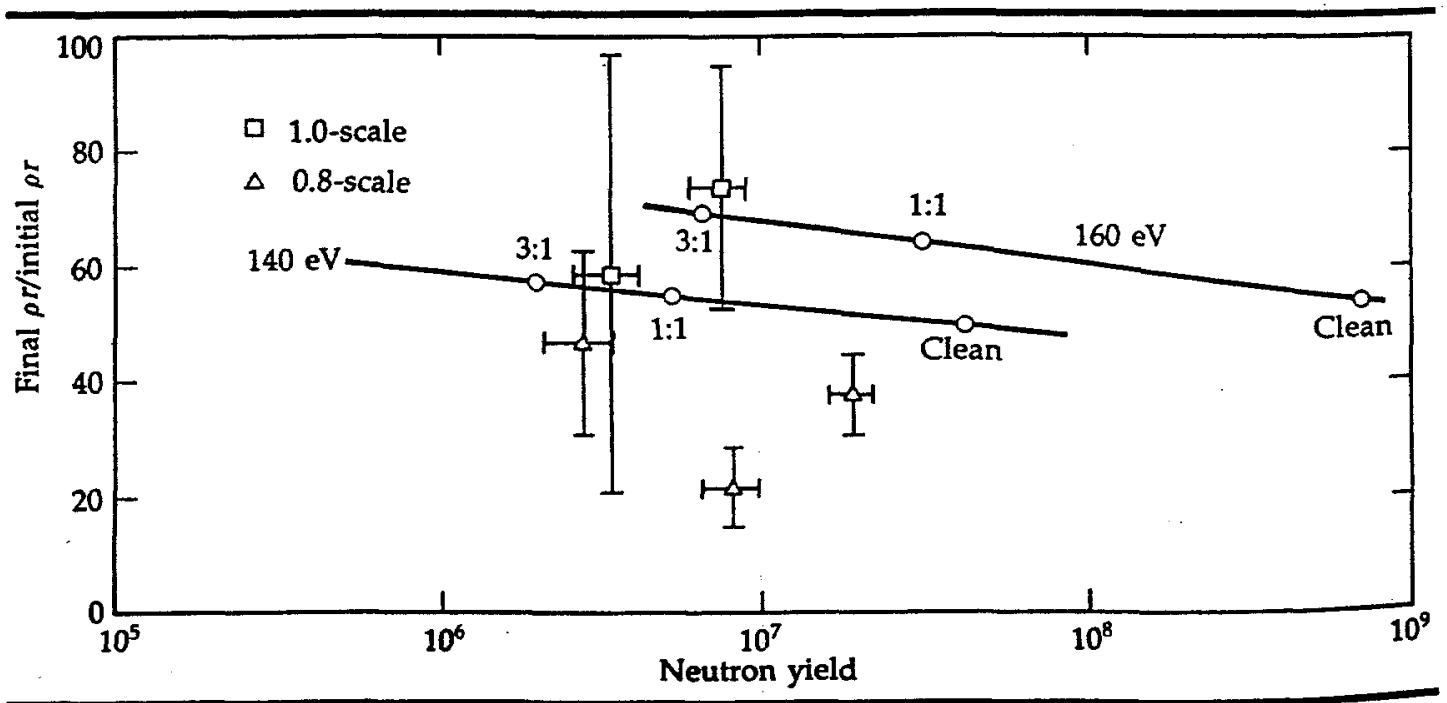


Analysis of Dante Data

model degrades the yield of the 0.8 -scale hohlraums more than that of the 1.0-scale hohlraums by mixing in more glass, with the result that the predicted $\rho r$ values are substantially higher for the 0.8 -scale hohlraums than for the 1.0-scale hohlraums, not lower. Consequently, we think that pure mix is not likely to be the reason for the low yields of our capsules.

\section{Conclusions}

Two of the goals of our Novette compression series were to field gas-filled capsules that would achieve high density $(>100 \times)$ and high temperature $(\sim 1 \mathrm{keV})$; and to field a combination of hohlraums and diagnostics that would allow us to determine what went wrong if we had problems. The pusher $\rho r$ measurements fairly conclusively indicate that we achieved reasonably high densities, probably in the range of $100 \times$. However, we did not achieve high temperatures; the neutron yields indicate that our peak fuel temperature was closer to $550 \mathrm{eV}$ than to $1 \mathrm{keV}$.

Our hohlraum experiments, combined with our recent advances in hohlraum modeling, have allowed us to understand many details of the hohlraum, especially those details that affect capsule drive symmetry in the polar direction. We have gathered enough information to intelligently plan another round of experiments that could tell us, for example, whether the lasercone interaction is axisymmetric and whether our model is correct when it predicts that an 0.8-scale hohlraum with a 925- $\mu \mathrm{m}$-long secondary would provide exceptionally good flux symmetry. Unfortunately, the story ends here. The Novette shutdown schedule does not allow us the few more shots we would need to conduct a new series of experiments.

Author: L. J. Suter

\section{Analysis of Dante Data}

We have developed two graphical techniques, channel-ratio plots and $T_{r}$-channel plots, that enable us to access more of the information contained in our Dante data. Usually, these techniques corroborate the
Dante temperature values (the normal product of Dante analysis), giving us greater confidence in these values. Occasionally, the techniques also produce new information that could not be extracted by the usual analyses. The two graphical techniques are described below.

\section{Channel-Ratio Plots}

A channel-ratio plot is a scaling tool that helps us estimate how temperature changes when we change the experiment. This type of plot is generated by plotting the ratio of Dante voltages for two experiments against the channel number. A typical plot is shown in Fig. 4-49, in which the Dante $\mathrm{H}$ voltage measured on a 0.8-scale full Cairn (shot No. 93121905) is divided by the Dante $\mathrm{H}$ voltage measured on a 1.0-scale full Cairn (shot No. 93120807), and the resulting ratio is plotted by channel number (see the article "Results of the Novette Implosion Experiments," earlier in this section.)

We use channel-ratio plots by comparing the experimental ratio against ratios that would be produced by blackbody temperatures. For example, shown on Fig. 4-49 are the theoretical channel-ratio plots for blackbodies at 155 and $130 \mathrm{eV}, 150$ and $130 \mathrm{eV}$, and 145 and $130 \mathrm{eV}$. We use the channel-ratio plots by seeing which of the theoretical curves best fits the data curve; this best-fit theoretical curve then gives us an estimate of the temperature ratio for the experiment. In the case shown in Fig. 4-49, it would appear that when we go from a 1.0- to a 0.8-scale Cairn, the Dante temperature rises by 20 to $25 \mathrm{eV}$.

Channel-ratio temperatures usually agree with regular Dante temperatures. In the example of Fig. 4-49, the single-point Dante temperatures were 157 and $136 \mathrm{eV}$, close to the channel-ratio estimate. Note, however,

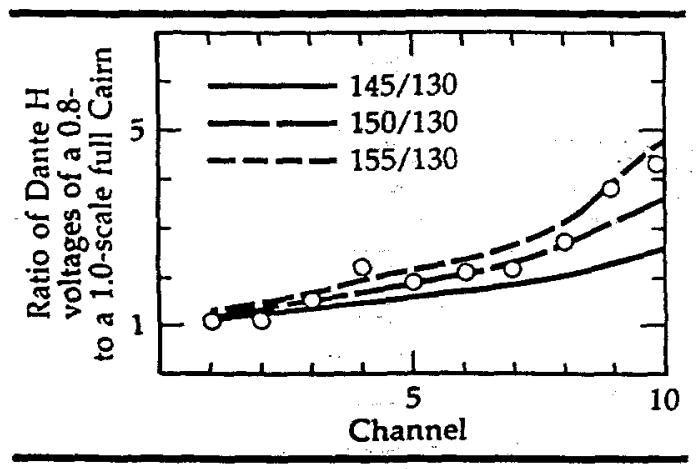

Fig. 4-49. Typical channel-ratio plot comparing 0.8 - and 1.0-scale full-Cairns. 
Fig. 4-50. Channelratio plot from two LASNEX TDG calculations.

Fig. 4-51. (a) Geometry of the cone experiments. (b) Geometry of the double-disk Dante experiment.

Analysis of Dante Data

that the temperatures we infer from channel-ratio plots are not unique. All of the temperature information is carried in the higher energy Dante channels, which cover the tail of the spectrum, so the ratio in a given channel will be roughly proportional to

$e^{\operatorname{lin}(1 / T-1 / T)}$

where $h v$ is the energy that characterizes a given channel. Thus, all combinations of temperatures that produce the same $1 / T-1 / T$ will match the experiment. Channel-ratio plots will not tell us absolute temperatures.

At first, it seems somewhat surprising that we can compare the channel-ratio plots with blackbody curves, since the spectra that Dante measures are not blackbody.
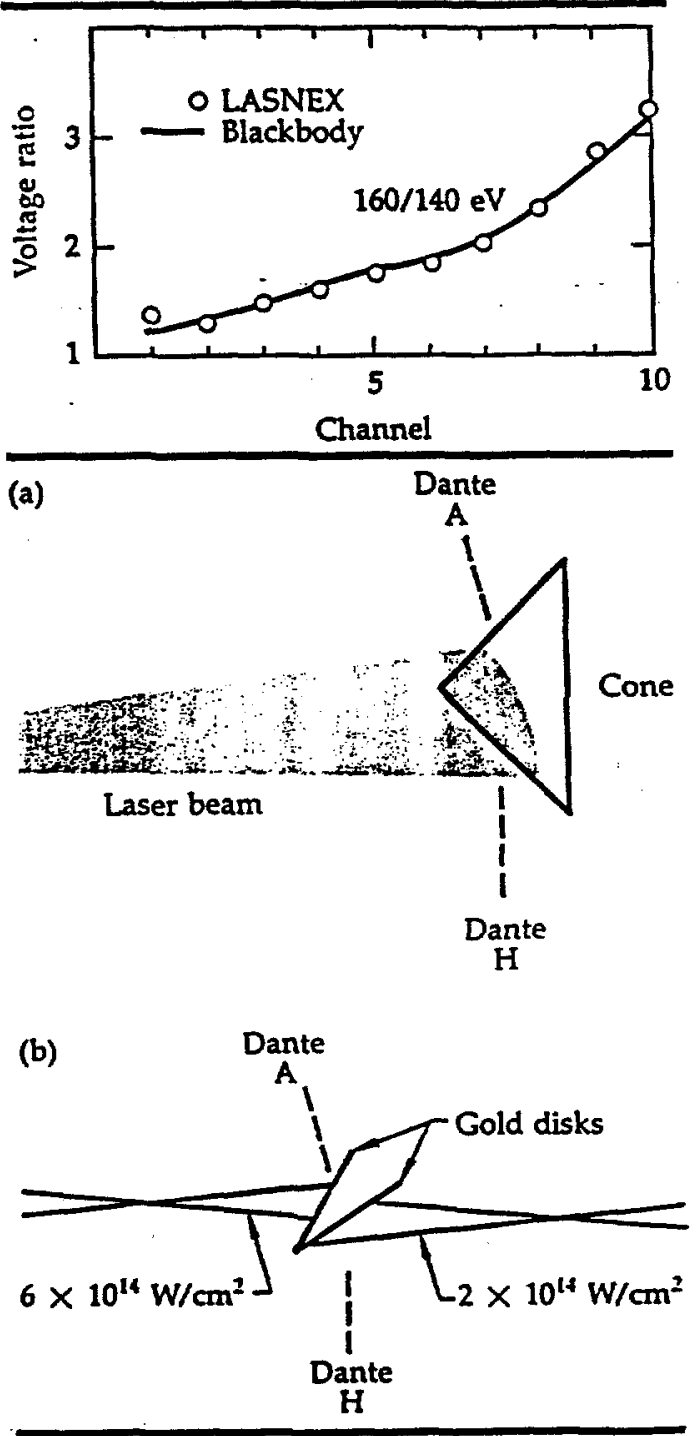

However, LASNEX simulations indicate that this technique makes sense. Figure 4-50 shows a channel-ratio plot obtained from LASNEX calculations in which we simulated two gold hohlraums that were heated by laser-generated radiation sources and that produced nonblackbody wall emissions. We used our LASNEX postprocessor, TDG, to simulate the Dantes viewing the walls, and we then used the calculated Dante voltages to make the channel-ratio plot for the hohlraums.

Independent LASNEX TDG calculations said that the total-flux temperatures from the two gold walls were 160 and $140 \mathrm{eV}$. When we made channel-ratio plots for blackbodies at these temperatures, we found that the curve was in good agreement with the LASNEX TDG curve, as shown in Fig. 4-50. The conclusion we draw from this, and similar calculations, is that, even though the measured spectrum is not blackbody, individual parts of the spectrum (analageus to photon bins) vary with emission temperature much like a blackbody. Thus, hohlraum-temperature measurements from the tail of the spectrum can make sense.

In the example of Fig. 4-49, we used channel-ratio plots in a situation where we knew the emission areas, which were defined by the Dante holes. It turns out that channel-ratio plots can also be used to estimate the temperatures of sources when the emission areas are not known. In this situation, we take channel ratios against a known source.

One example of this use of channel-ratio plots is shown in Fig. 4-51(a), in which a $600-\mu \mathrm{m}$ bare scttering cone is illuminated by a $400-\mu \mathrm{m}$ laser spot. Because the laser beam was misaligned, we could not know how much irradiated area was actually seen by the Dante $A$ and Dante $H$ in each of the tests.

We were able to estimate the emission temperatures of the two cones by making channel-ratio plots against a standard double-disk shot (shot.No. 93112811), as shown in Fig.4-51(b). In this double-disk experiment, Dante $\mathrm{H}$ saw a gold disk that was heated by a pulse of $\sim 2.4 \times 10^{14} \mathrm{~W} / \mathrm{cm}^{2}$; this disk radiated at 170 to $180 \mathrm{eV}$, assuming 70 to $80 \%$ absorption and $50 \%$ conversion efficiency. Dante $A$ saw a gold disk that was heated by a pulse of $6 \times 10^{14} \mathrm{~W} / \mathrm{cm}^{2}$; 
this disk radiated at $\sim 215 \mathrm{eV}$, assuming $70 \%$ absorption and $45 \%$ conversion efficiency. When we made channel-ratio plots of Dante A to Dante $H$ for this experiment (a less-certain process than making ratios of the same Dantes), we found that the disk viewed by Dante $A$ was, in fact, $\sim 45 \mathrm{eV}$ hotter than the disk viewed by Dante $\mathrm{H}$, which is consistent with our estimates (see Fig. 4-52).

Channel-ratio plots indicate that parts of our cones were hot and other parts were cold. Figure 4-53 shows a channel-ratio plot comparing the Dante $H$ that viewed cone 1 (shot No. 93091404) with the Dante $H$ that viewed the colder of the two gold disks. A comparison of this plot against blackbody curxes indicated that cone 1 was radiating at $\sim 220 \mathrm{eV}$, assuming the disk was radiating at $175 \mathrm{eV}$.

Because the cone-emitting area is different from the disk-emitting area, the ratio at the lower end of the curve for cone 1 in Fig. $4-53$ is substantially different from 1; for equal-emission areas, we would expect this ratio to be a little larger than 1 . The lower end of the curve contains most of the area information, while the upper end of the curve contains the temperature information. To estimate the temperature ratio, we had to scale the theoretical blackbody ratios with an arbitrary multiplier that we consider to be close to the area ratio. Thus, the technique of using channel-ratio plots with uncertain emission areas involves a twoparameter fit area ratio and $1 / T-1 / T^{2}$. This two-parameter fit is acceptable, since we have 10 points defining the curve.

Cone 1

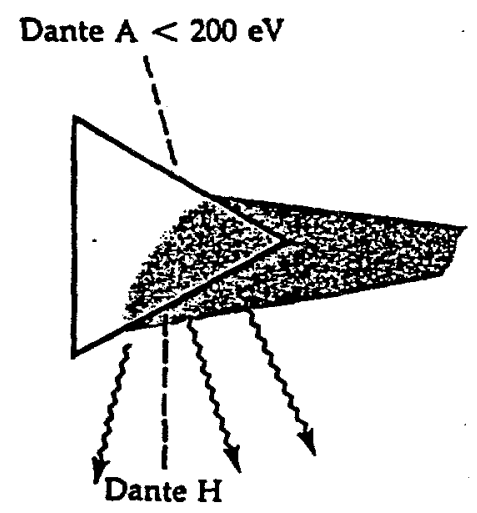

210 to $220 \mathrm{eV}$

$1.7 \times 10^{15} \mathrm{~W} / \mathrm{cm}^{2}$ incident energy

$\sim 30$ to $70 \%$ absorption
Figure 4-53 also shows the channelratio plot of values from the Dante $H$ that viewed cone 2 (shot No. 93102411) and the Dante $\mathrm{H}$ that viewed the colder of the gold disks. In this case, the ratio is flat, indicating that the side of the cone viewed by

Dante $H$ was quite cold on this experiment.

Our analyses of the Dante channelratio plots has led to a more complete picture of the cone experiments. Figure 4-54
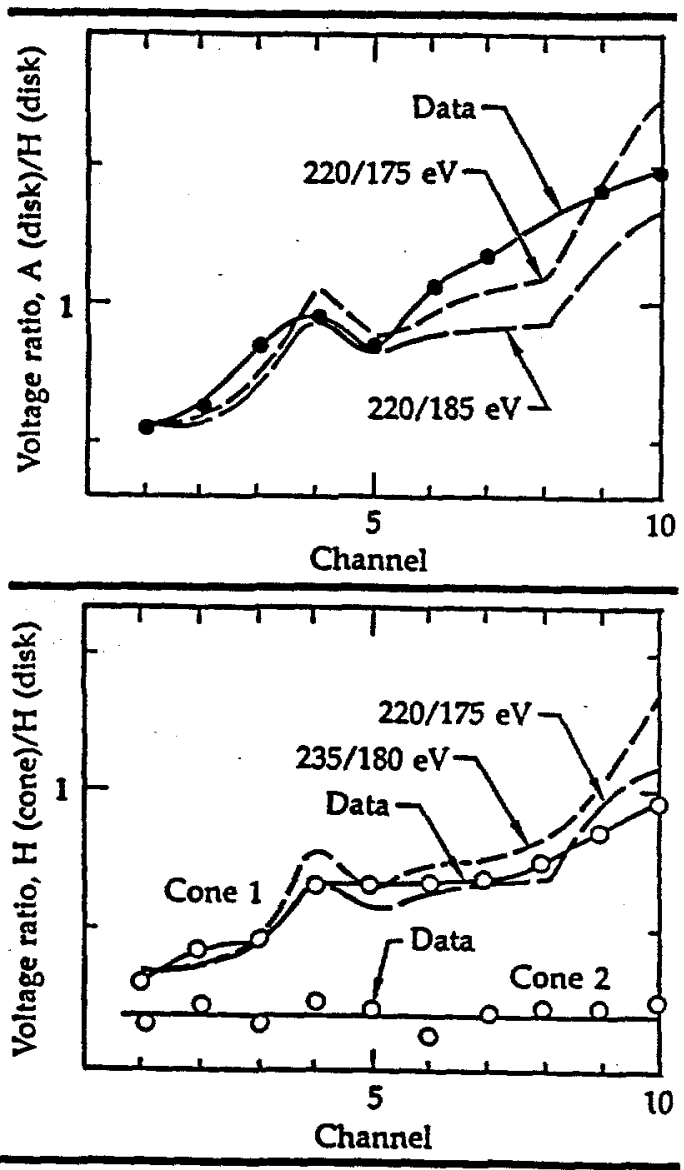

Cone 2

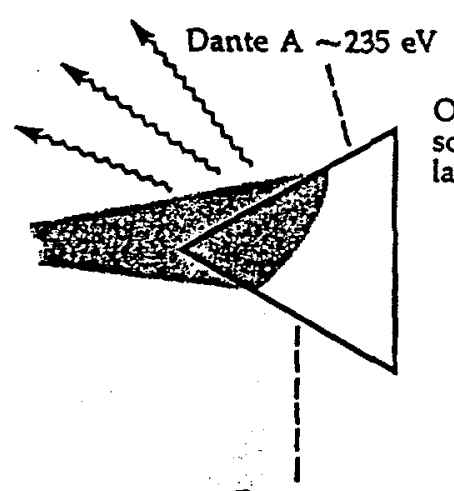

Observed scattered laser light
Fig. 452 Channelratio plot from the double-disk Dante experiment.
Fig. 4-53. Channelratio plots of Dante $H$ viewing cone 1 to Dante $\mathrm{H}$ viewing the colder disk and of Dante $\mathrm{H}$ viewing cone 2 to Dante $H$ viewing the colder disk.

Fig. 4-54. Summary of results obtained by channel-ratio plots for the cone experiments. $\sim 180 \mathrm{eV}$

$2.1 \times 10^{15} \mathrm{~W} / \mathrm{cm}^{2}$ incident energy $\sim 60$ to $80 \%$ absorption 
Fig. 4-55. $T_{r}$-channel plot for 0.8- and 1.0 seale full-Cairns.

Fig. 4-56. (a) $T_{\text {- }}$ channel plot for LASNEX radiationheated hohlraums. (b) Trechannel plot for dinectly irradiated LASNEX hohlraum.
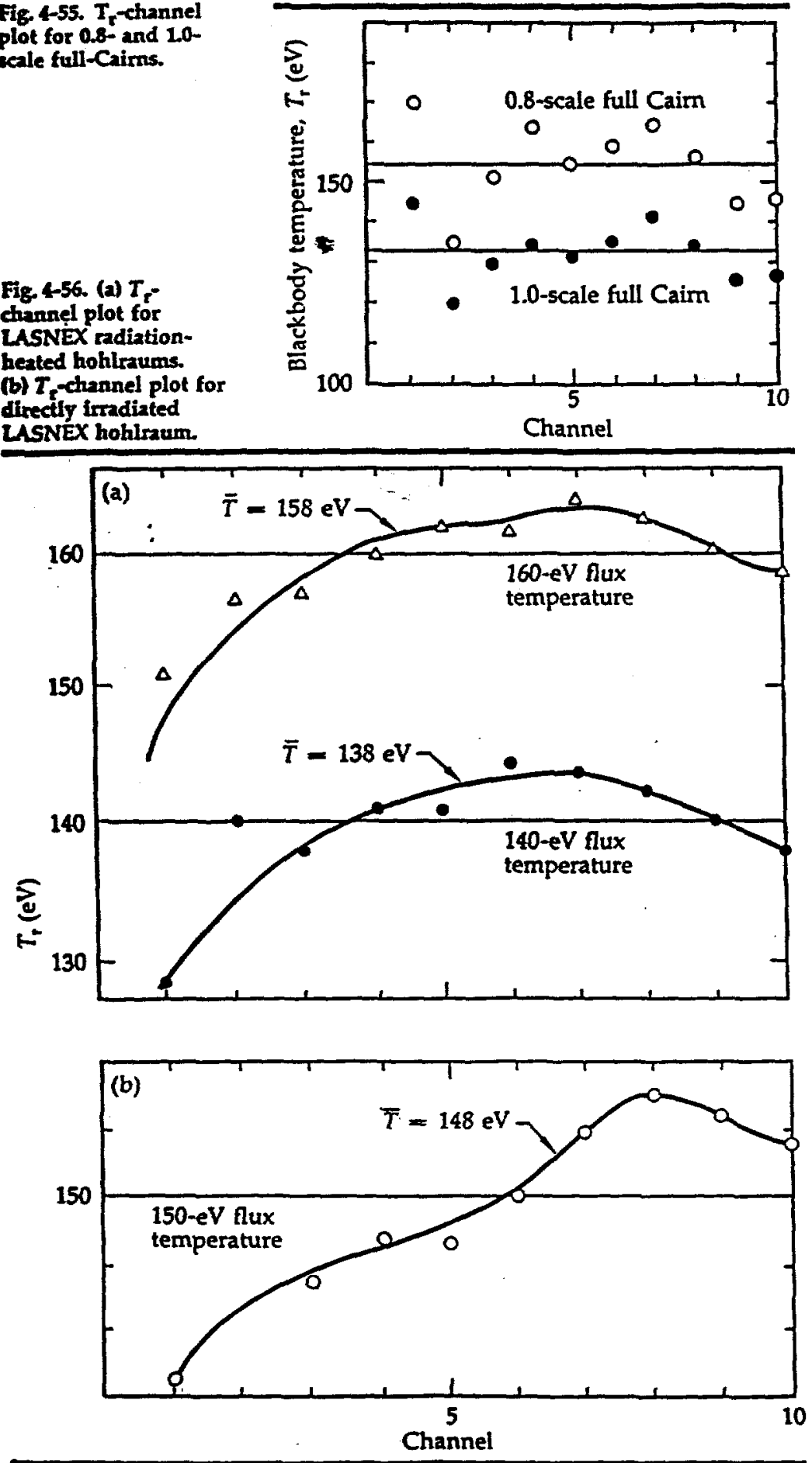

Analysis of Dante Data

summarizes our analysis of the cone experiments. The Dantes showed that one side of each cone was hot and one side was cold. Independent measurements showed that most of the scattered laser light was coming from the hot side of the cones. The radiation temperatures we inferred from the channel-ratio plots are consistent with: - Nominal spot size (400 $\mu \mathrm{m}), 70 \%$ absorption, and $30 \%$ conversion efficiency.

\section{$T_{\mathrm{r}}$-Channel Plots}

The second graphical technique for handling Dante data is what we call $T_{r}$-channel plots, where $T_{r}$ stands for radiation temperature. The prescription for generating these plots is to take the peak Dante voltage for a channel, normalize the voltage to our standard $400-\mu \mathrm{m}$ hole, and look up in a table which blackbody temperature $\left(T_{r}\right)$ would produce that voltage. The value for $T_{r}$ is then plotted against the channel number.

Figure 4-55 shows. $T_{\mathrm{r}}$-channel plots for 0.8- and 1.0-scale full-Caims (shot Nos. 93121905 and 93120807, respectively): This figure permits us to make four points about $T_{r}$-channel plots. First, these plots produce temperatures that usually agree with singlepoint Dante temperatures. From the plots in Fig. 4-55, we infer temperatures for the 0.8and 1.0-scale Caims of $\sim 155$ and $133 \mathrm{eV}$, respectively, which are close to the singlepoint Dante temperatures of 157 and $136 \mathrm{eV}$, respectively.

Second, the plots clearly show the effects of scaling. The 0.8-scale Caim appears to be $\sim 20$ to $25 \mathrm{eV}$ hotter than the 1.0-scale

Cairn. This temperature differential also agrees with our channel-ratio analysis of the same experiments, shown in Fig. 4-49.

Third, the plots provide us with crude spectral information. Note that the two plots in Fig. 4-55 have the same nonblackbody shape, even though they differ by $>20 \mathrm{eV}$. This conformity of shapes is consistent with our earlier contention that different parts of the spectrum vary with emission temperature in the same manner as a blackbody. 
Analysis of Dante Data

Fourth, we do not need to make any assumptions about $x$-ray duration to arrive at a temperature estimate.

LASNEX calculations indicate that $T_{r}$-channel plots make good sense. Figure 4-56(a) shows the $T_{r}$ channel plots that we generated with LASNEX TDG for the two hohlraum simulations described earlier (see discussion above regarding Fig. 4-50). The two horizontal lines through the data in Fig. 4-56(a) are the TDG-inferred total flux temperatures from the walls of the hohlraums. The spectra are nonblackbody, being cooler than the average flux temperature at both the low and high ends, and the calculated spectral shapes agree with the shapes of actual data shown in Fig. 4-55, except for channel 1. In channel 1, the data are consistently high, perhaps indicating that something is wrong with the channel.

One handy result of the LASNEX analysis is that simply averaging the values for $T_{r}$ in the plots gives a temperature that is very close to the total flux temperature. There is no fundamental reason for this result it just works out that way when the Dante channels are judiciously chosen. In Fig. 4-56(a), the average $T_{\mathrm{r}}$ values are 158 and $138 \mathrm{eV}$, close to the $160-$ and $140 \mathrm{eV}$ flux temperatures. Figure 4-56(b) shows the $T_{\mathrm{r}}$-channel plot for non-LTE gold that has been directly irradiated by laser energy, producing a different spectrum than those just discussed. Again, however, the average $T_{r}$ of $148 \mathrm{eV}$ for the directly irradiated gold is close to the flux temperature of $150 \mathrm{eV}$.

The $T_{\mathrm{r}}$-channel plots have three working advantages over the more formally correct alternative, a Dante spectrum produced from an unfold:

- Temperature information is displayed in a way that is easier to understand and that makes it easier to compare experiments.

- Spectral variations are crudely indicated. and the results can be easily compared with LASNEX TDG results.

- A good approximation to the total flux temperature is obtained, according to LASNEX calculations; furthermore, a complement to the total-flux temperature is obtained, since $T_{r}$ makes no assumptions about pulse duration.

$T_{r}$-channel plots must be corrected for two effects-instrumental time response and hole closure- that act to produce an observed peak voltage that is lower than
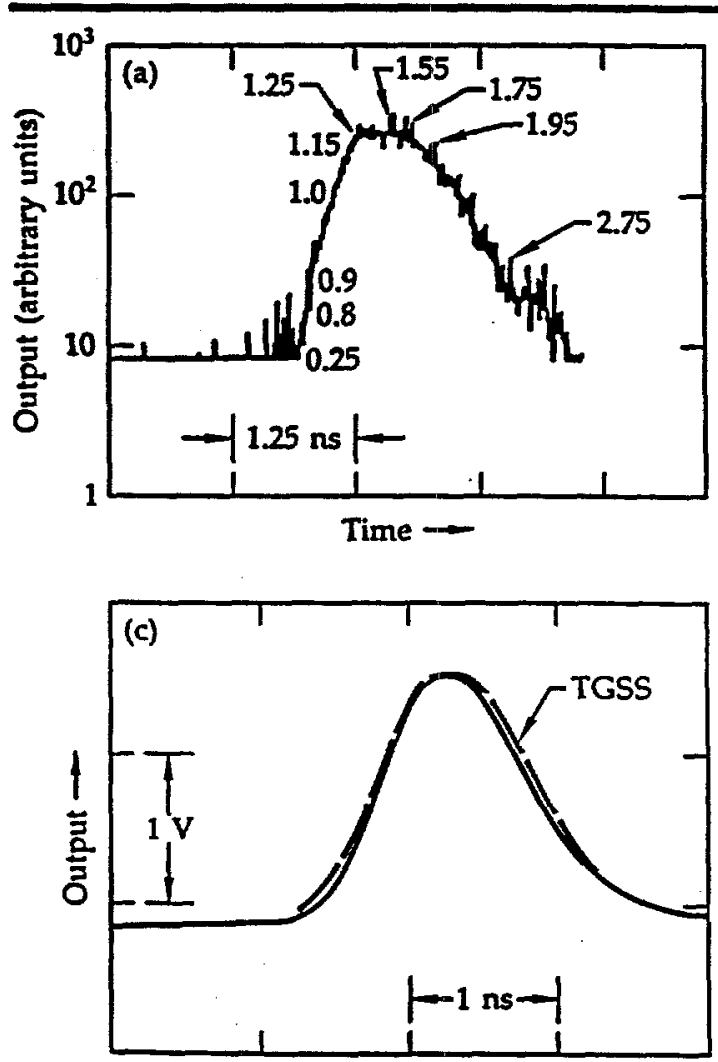

Time -
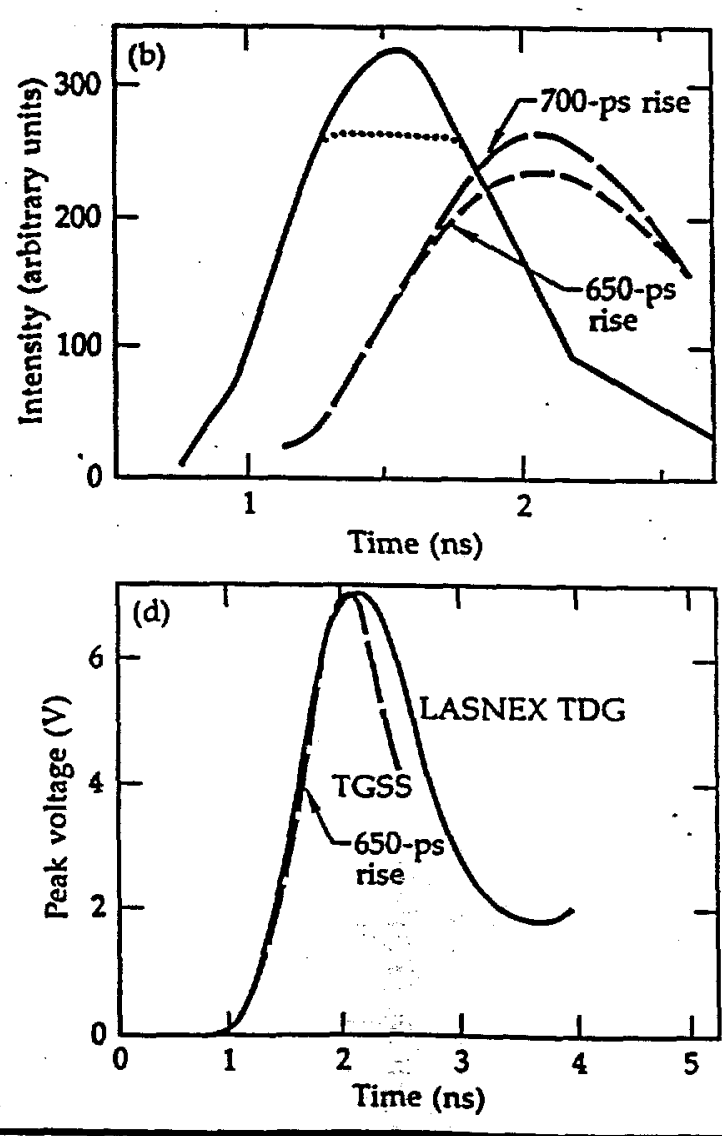

Fig. 4-57. (a) TGSS data vs time at $600 \mathrm{eV}$. (b) Linear plots of TCSS data before (solid and dotted) and after (dashed) being. convolved. (c) Comparison of convolved TCSS data with the corresponding Dante channel. (d) TGSS has FWHM shorter than LASNEX predicts, possibly due to hole closure. 
Fig 4-58. $T_{\mathrm{r}}$-channel plots of raw and corrected data for the 0.8 and I.0-scile Ciirns.

\section{Analysis of Dante Data}

the true peak voltage. Our analysis indi= cates that these two effects require a correction of $\sim 8 \mathrm{eV}$.

Figure 4-57(a) is a lineout from the transmission-grating streak camera (TGSS) (Ref. 38), showing $x$-ray output from the Dante A hole of a 1.0-scale Caim vs time (shot No. 93102615). The time response for the TGSS is $\sim 20 \mathrm{ps}$, and the lineout was taken at $600 \mathrm{eV}$, which corresponds to the chromium channel of the Dante.

The data are replotted on a linear scale as the solid curve in Fig. 4-57(b); because the data are noisy, we drew a dotted line that brackets the uncertainty. Figure 4-57(b) also shows our estimate of what happens to the time profile when it is measured by Dante. The two dashed curves show the TGSS data convolved with a 600-ps Gaussian response function; the effect of the instrumental time response is to reduce the peak voltage by only 10 to $20 \%$. This conclusion is validated by Fig. 4-57(c), in which convolved TGSS data, which have been peak

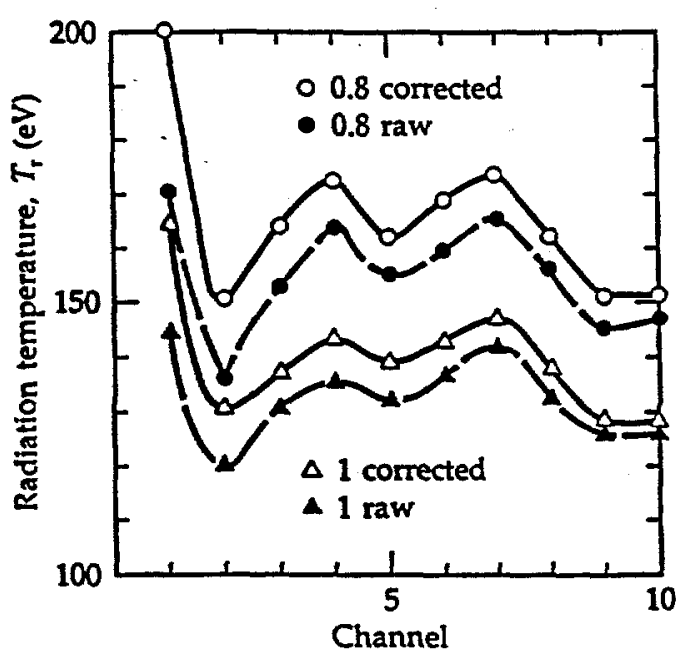

normalized to the trace, are compared with the raw Dante chromium data. As may be seen, the convolved shape matches the observed shape quite well.

Besides the reduction in peak voltage due to instrumental response, there may also be a reduction in this voltage due to hole closure. Support for a hole-closure effect appears in Fig. 4-57(d), which compares the $600-\mathrm{eV}$ TGSS data with a LASNEX TDG calculation of the output from a laserheated hohlraum. The peak-normalized TGSS data have a smaller FWHM, presumably due to hole closure. A quantitative estimate of the effects of hole closure can be obtained from our analysis of the NPIRE I experiments. ${ }^{39}$ We concluded that hole dosure reduced the peak voltage by $\sim 15 \%$ in this series of experiments, which used about the same pulse length, radiation temperature, and Dante hole size as were used with our current 1.0-scale Cairns.

The net correction for both hole closure and instrumental response appears to be a reduction of $\sim 25 \%$ in the peak voltage. Figure 4-58 is a $T_{r}$-channel plot showing raw and corrected temperatures for our 0.8 and 1.0-scale Cairns. The average temperature of the corrected data is $\sim 8 \mathrm{eV}$ hotter than the raw data.

Figure 4-58 demonstrates the well-known fact that spectral-tail measurements are more sensitive indicators of temperature than are low-energy measurements. At the low end of the spectrum, where $d V / d T_{r}$ is smallest, the corrections amount to 10 to $15 \mathrm{eV}$. As we approach the tail of the spectrum, where the output voltage changes rapidly with temperature, the correction drops to 3 to $5 \mathrm{eV}$.

Author: L. J. Suter 